\title{
Specific interactions between rifamycin antibiotics and water influencing ability to overcome natural cell barriers and range of antibacterial potency
}

K. Pyta, ${ }^{a}$ A. Janas, ${ }^{a}$ N. Skrzypczak, ${ }^{a}$ W. Schilf, ${ }^{b}$ B. Wicher,${ }^{c}$ M. Gdaniec, ${ }^{a}$ F. Bartl, ${ }^{d}$ P. Przybylski*a

aFaculty of Chemistry, Adam Mickiewicz University, Uniwersytetu Poznanskiego 8, 61-614 Poznan, Poland

${ }^{b}$ Institute of Organic Chemistry, Polish Academy of Science, Kasprzaka 44/52, 01-224 Warsaw, Poland

'Department of Chemical Technology of Drugs, Poznan University of Medical Sciences,

Grunwaldzka 6, 60-780 Poznan, Poland

${ }^{d}$ Humboldt-Universität zu Berlin, Lebenswissenschaftliche Fakultät, Germany, Institut für Biologie, Biophysikalische Chemie, Invalidenstr. 4210099 Berlin

*corresponding authore-mail: piotrp@amu.edu.pl 
1. STRUCTURE AND ATOMS NUMBERING OF STUDIED COMPOUNDS …...................S4

2. GENERAL EXPERIMENTAL

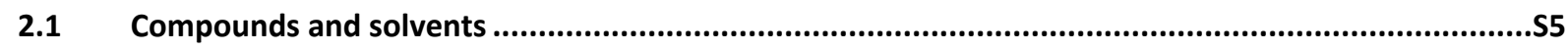

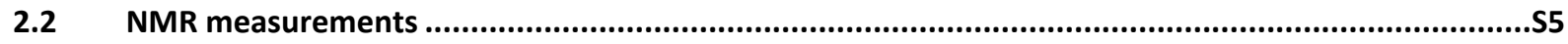

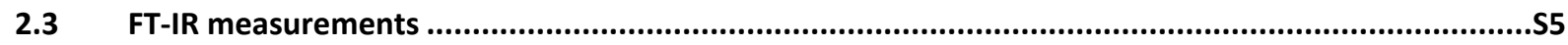

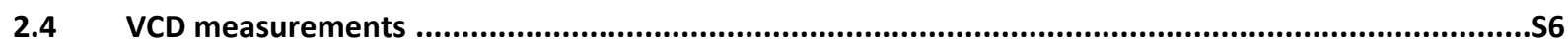

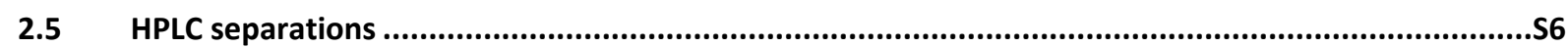

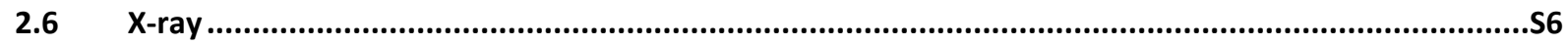

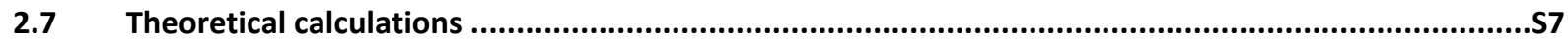

3. FIGURES

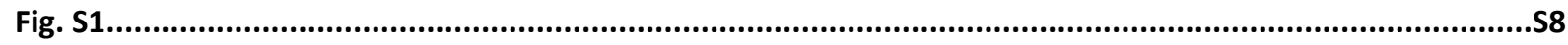

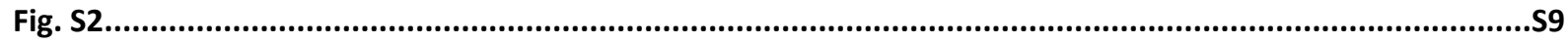

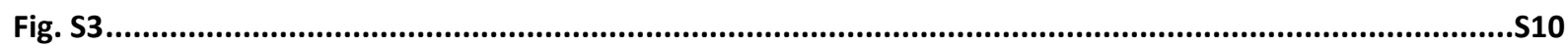

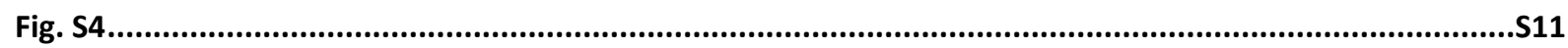

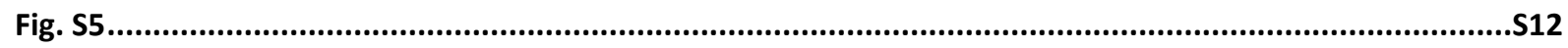

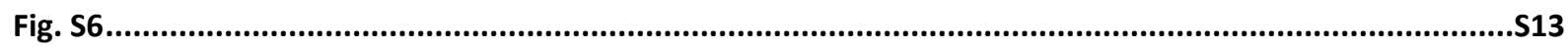

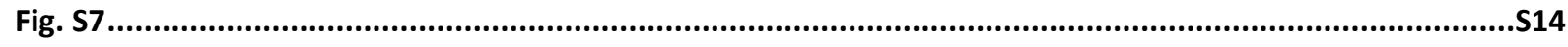

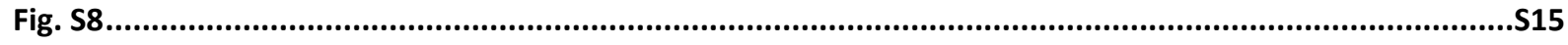

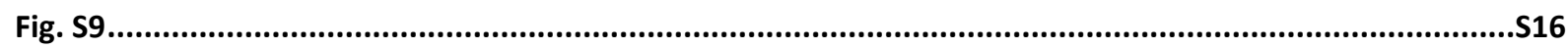

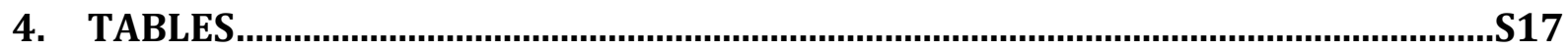

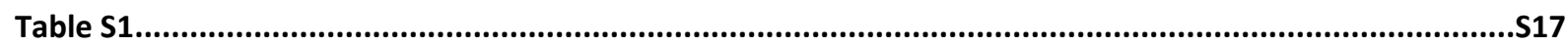

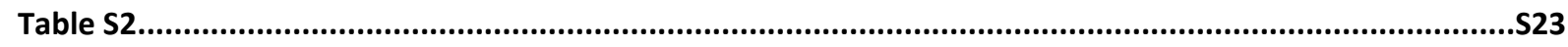

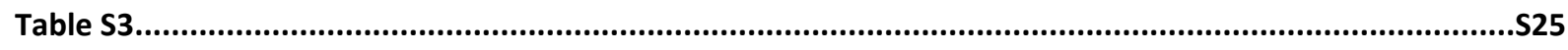

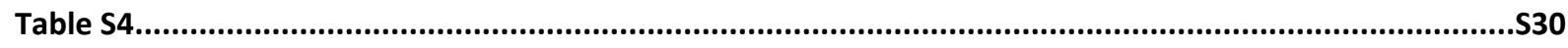

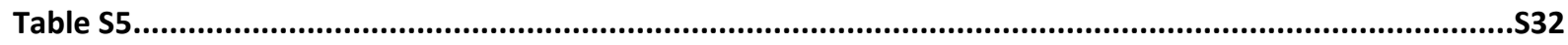

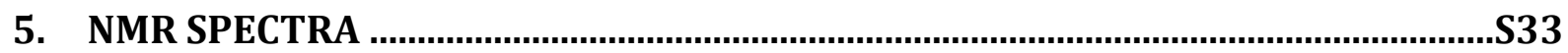




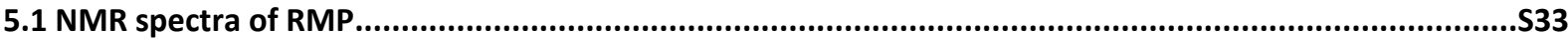

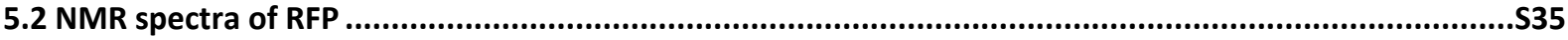

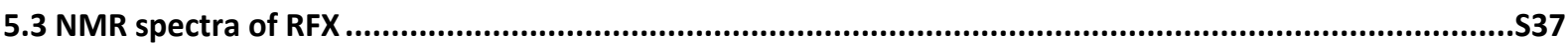

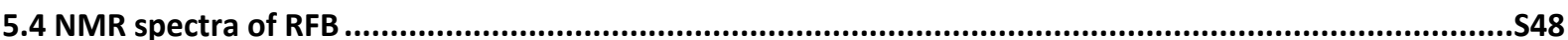

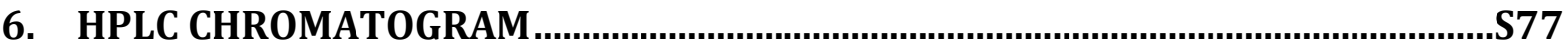

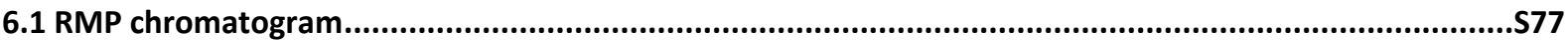

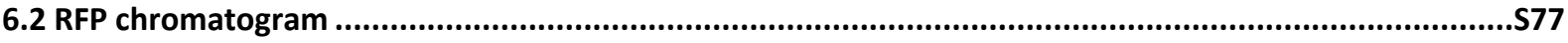

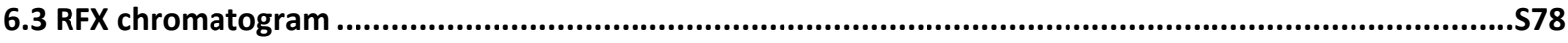

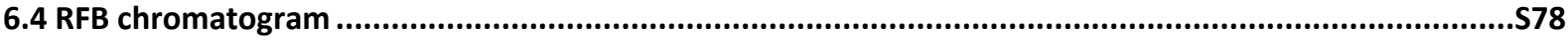


1. Structure and atoms numbering of studied compounds
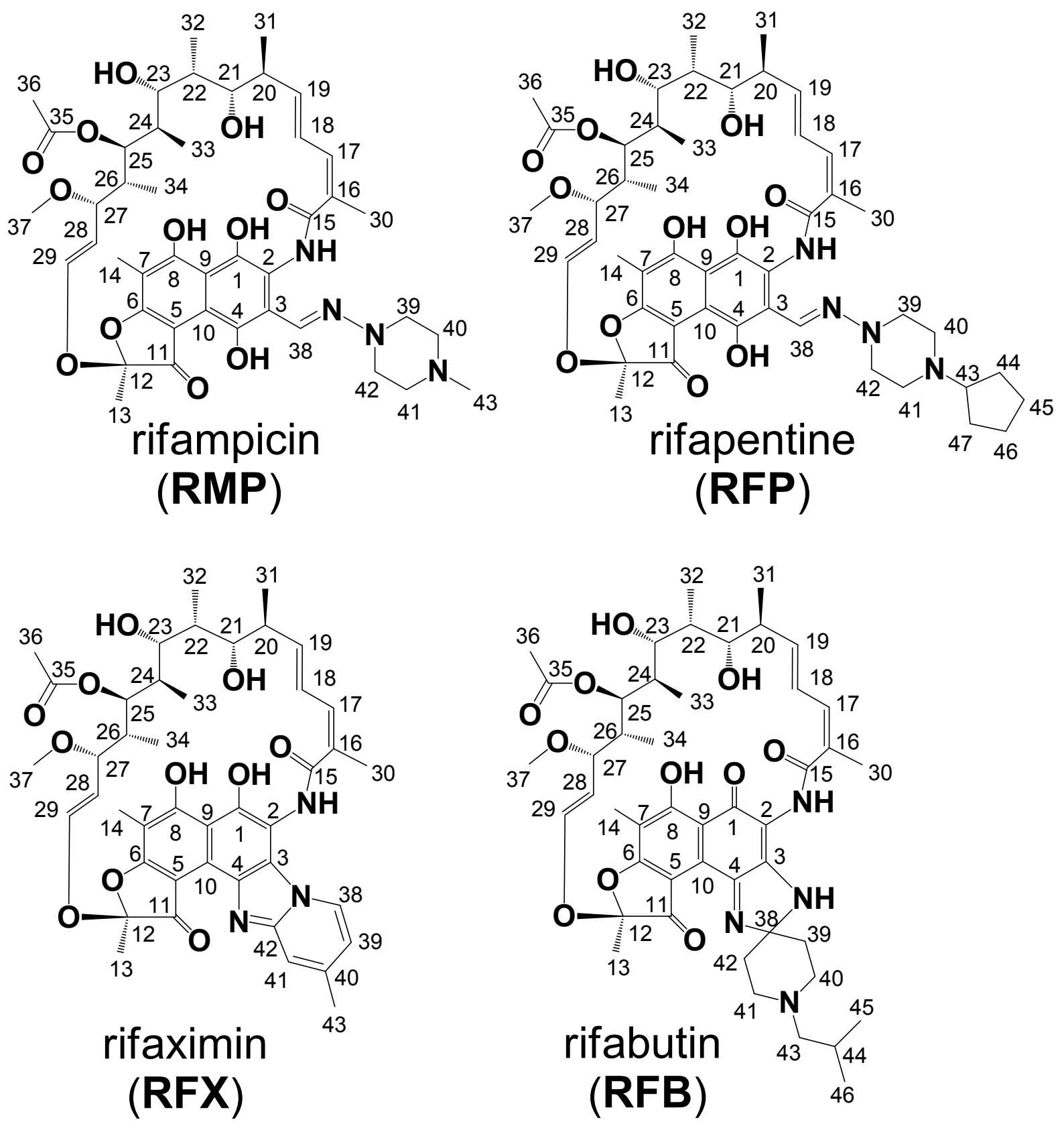


\section{General Experimental}

\subsection{Compounds and solvents}

All studied compounds were purchased from Sigma -Aldrich: Rifampicin (RMP) [CAS: 13292-46-1], Rifapentine (RFP) [CAS: 61379-65-5], Rifaximin (RFX) [CAS: 80621-81-4], Rifabutin (RFB) [CAS: 72559-06-9]. The purity of all antibiotics has been checked using HPLC methods.

All solvents used to measured NMR, FT-IR and VCD spectra were purchased from Sigma -Aldrich: acetonitrile- $\mathrm{d}_{3}$ [CAS: 2206-26-0], dimethyl sulfoxide- $\mathrm{d}_{6}$ [CAS: 2206-27-1], chloroform-d [CAS: 865-49-6], pyridine- $\mathrm{d}_{5}$ [CAS: 7291-22-7], deuterium oxide [CAS: 778920-0]; and from Baker: water [CAS: 7732-18-5]

\subsection{NMR measurements}

The ${ }^{1} \mathrm{H}$ and ${ }^{13} \mathrm{C}$ NMR measurements of RMP, RFP, RFX and RFB were performed in $\mathrm{CDCl}_{3}, \mathrm{CD}_{3} \mathrm{CN}, \mathrm{DMSO}-\mathrm{d}_{6}$, DMSO- $\mathrm{d}_{6}: \mathrm{CD}_{3} \mathrm{CN}(1: 1), \mathrm{CD}_{3} \mathrm{CN}+\mathrm{H}_{2} \mathrm{O}, \mathrm{DMSO}-\mathrm{d}_{6}+\mathrm{H}_{2} \mathrm{O}$ and pyridine- $\mathrm{d}_{5}$ Bruker Avance III $600 \mathrm{MHz}$.

For Bruker Avance $600 \mathrm{MHz}$ spectrometer: the operating frequencies for ${ }^{1} \mathrm{H}$ measurements was $600.08 \mathrm{MHz}$; pulse width corresponding to the flip angle of $45^{\circ}$; spectral width swh $=$ $9842.5 \mathrm{~Hz}$; acquisition time at $=0.2 \mathrm{sec}$; relaxation delay $\mathrm{d}_{1}=1.0 \mathrm{~s} ; \mathrm{T}=293.0 \mathrm{~K}$, TMS was used as the internal standard. No window function or zero filling were used. Digital resolution was $0.2 \mathrm{~Hz} /$ point. ${ }^{13} \mathrm{C}$ NMR spectra were recorded at the operating frequency $150.454 \mathrm{MHz}$; pulse width corresponding to the flip angle of $60^{\circ} ; \mathrm{sw}=19000 \mathrm{~Hz}$; at $=1.8 \mathrm{~s} ; \mathrm{d}_{1}=1.0 \mathrm{~s} ; \mathrm{T}=$ 293.0 K and TMS as the internal standard. Line broadening parameters of 0.5 or $1 \mathrm{~Hz}$ were applied.

The ${ }^{1} \mathrm{H}$ and ${ }^{13} \mathrm{C}$ NMR resonances in solution (collected in Tables S1-S4) were unambiguously assigned on the basis of the HMBC, HSQC, COSY and NOESY correlation spectra.

\section{$2.3 \quad$ FT-IR measurements}

The FT-IR spectra of RMP, RFP, RFX and RFB were recorded in $\mathrm{CDCl}_{3}, \mathrm{CD}_{3} \mathrm{CN}$, $\mathrm{CD}_{3} \mathrm{CN}+\mathrm{H}_{2} \mathrm{O}$, DMSO- $\mathrm{d}_{6}+\mathrm{H}_{2} \mathrm{O}$ solution. All FT-IR spectra were recorded on an iS50 FT-IR spectrophotometer (Thermo, Nicolet, US) equipped with a DTGS detector and two-columnar purge gas generator (Parker, Balston, US); resolution $1 \mathrm{~cm}^{-1}$, NSS $=150$, range 4000-400 $\mathrm{cm}^{-1}$. The Happ-Genzel apodization function was used. 


\subsection{VCD measurements}

The VCD spectra of RFB were recorded in $\mathrm{CDCl}_{3}$ and DMSO- $\mathrm{d}_{6}+\mathrm{H}_{2} \mathrm{O}$ solution. All FTIR spectra were recorded on an FVS-6000 Vibrational Circular Dichroism Spectrometer (Jasco) equipped with a MCT_V detector; resolution: $4 \mathrm{~cm}^{-1}$, range: $2000-850 \mathrm{~cm}^{-1}$.

\subsection{HPLC separations}

HPLC separations were performed with a Dionex Ultimate 3000 equipped with LPG-3400 SD gradient pump using Thermo GOLD C18 150×4.6 mm $(5 \mu \mathrm{m})$ Accucore XL column, TCC-3000SD thermostat to columns (column temp. equal $25^{\circ} \mathrm{C}$ ) and Dionex VWD-3400RS variable wavelength UV-vis detector. The flow rate was $1 \mathrm{~mL} / \mathrm{min}$ with injection volumes of $10 \mu \mathrm{L}$. The analytical wavelength was $\lambda_{\max }=260 \mathrm{~nm}$ (RMP, RFP and RFX) and $220 \mathrm{~nm}$ (RFB). The $0.01 \mathrm{M}$ solution of ammonium acetate in $\mathrm{H}_{2} \mathrm{O}: \mathrm{CH}_{3} \mathrm{CN}(1: 1)$ mixture as a buffer. For RMP, RFP and RFX mixture of $\mathrm{H}_{2} \mathrm{O}: \mathrm{CH}_{3} \mathrm{CN}(60: 40)$ as amobile phase was used and for RFB $\mathrm{H}_{2} \mathrm{O}: \mathrm{CH}_{3} \mathrm{CN}$ : buffer $(40: 50: 10)$ as amobile phase was used. RMP: $R t=3.832$ min. (94.38\%); RFP: $R t=6.240$ min. (98.38\%); RFX: $R t=11.282 \min$. (99.25\%); RFB: $R t=$ $6.693 \min .(95.89 \%)$

\subsection{X-ray}

Single crystals of rifampicin_methanol_trisolvate, $\mathrm{C}_{43} \mathrm{H}_{58} \mathrm{~N}_{4} \mathrm{O}_{12} \cdot\left(\mathrm{CH}_{3} \mathrm{OH}\right)_{3}$ were grown from anhydrous methanol. A suitable crystal was selected and diffraction data measured with a Kuma KM4CCD $\kappa$-geometry diffractometer using graphite monochromatized Mo Ka radiation. The crystal was kept at 100.0(2) K during data collection. The structure was solved with the SHELXS-1997 ${ }^{1}$ and refined with the SHELXL-2018 ${ }^{2}$ withn Olex2 ${ }^{3}$. H-atoms bonded to carbon atoms were placed in idealized positions. All $\mathrm{H}$ atoms of the $\mathrm{O}-\mathrm{H}$ and $\mathrm{N}-\mathrm{H}$ groups were located in difference Fourier maps, however for further refinement they placed in calculated positions and refined as riding on their carriers.

Crystal Data for $\mathrm{C}_{46} \mathrm{H}_{70} \mathrm{~N}_{4} \mathrm{O}_{15}(M=919.06 \mathrm{~g} / \mathrm{mol})$ : orthorhombic, space group $\mathrm{P} 2{ }_{1} 2_{1} 2_{1}$ (no. 19), $a=10.1242(6) \AA, b=13.9473(6) \AA, c=35.3927(17) \AA, V=4997.6(4) \AA^{3}, Z=4, T=$ $100.0(2) \mathrm{K}, \mu(\mathrm{MoK} \alpha)=0.091 \mathrm{~mm}^{-1}, D_{\text {calc }}=1.221 \mathrm{~g} / \mathrm{cm}^{3}, 19796$ reflections measured $\left(8.204^{\circ}\right.$ $\left.\leq 2 \Theta \leq 50.054^{\circ}\right), 8422$ unique $\left(R_{\text {int }}=0.1040, \mathrm{R}_{\text {sigma }}=0.3448\right)$ which were used in all calculations. The final $R_{1}$ was $0.0455(\mathrm{I}>2 \sigma(\mathrm{I}))$ and $w R_{2}$ was 0.0950 (all data).

Crystallographic data have been deposited at the Cambridge Crystallographic Data Centre with the deposition number CCDC 1883801. Copies of these data can be obtained free of 
chargé via www.ccdc.ca-m.ac.uk/conts/retrieving.html (or from the Cambridge Crystallographic Data Centre, 12 Union Road, Cambridge, CB2 1EZ, UK; Fax: +44 1223336 033; or e-mail: deposit@ccdc.cam.ac.uk).

\subsection{Theoretical calculations}

Calculations of the most energetically favourable structures of RMP, RFP, RFB and RFX (Figs. 4 and 5) were performed by DFT method - DGauss using the B88-LYP GGA energy functional with the DZVP basis sets (Scigress package version FJ. 2.4. EU 3.1.8). The starting models of rifamycin antibiotics and their hydrates were built using previously determined Xray structures of RMP analog and RMP pentahydrate. ${ }^{4,5}$ Structures of antibiotics were first optimized by MM3 and then recalculated by semi-empirical MO-G PM6 large molecule geometry algorithm, suitable for calculations of large systems comprising above 50 atoms. In the next step the most energetically favourable structures from PM6 calculations, which were in line with the spectroscopic data (NMR and IR), were selected to structural optimization by B88-LYP GGA DFT calculations with the energy gradient not exceeding $5 \mathrm{kcal} \mathrm{mol}^{-1}$ at one step (total optimization was achieved after $\sim 6000-\sim 11000$ steps). Docking of RFB and RFP antibiotics at molecular target (RNAP) were performed using X-ray structure of DNA dependent RNA polymerase (RNAP) from T. aquaticus ${ }^{6}$ - cube around binding site of dimensions $30 \AA \times 30 \AA \times 30 \AA$. Next the amino acids distanced from the binding site more than $30 \AA$ were removed and all $\alpha$-carbons of amino acids of the main polypeptide chains were locked. Docking of RFP and RFB at RNAP (Figs. 5d and e) were carried out by "dock into active site" mode on coordinates of rifampicin (RMP) using Scigress 3.1.8 package, Fujitsu. Next the intermolecular interactions between inhibitor molecule and the following key amino acids residues of RNAP's: Q393, F394, D396, R409 and S411 were assumed on the basis of Campbell et al. model of interactions found between rifampicin and RNAPc. Next, models of binding sites containing $\operatorname{RFP(A)}$ and $\operatorname{RFB(A)}$ antibiotics were initially optimized by MM3 and then finally recalculated using MO-G PM6 semi-empirical method using MOZYME algorithm dedicated to huge molecules (Fujitsu, Scigress package 3.1.8, 2008-2015) with the final result displayed in Figs. 5d and 5e. All calculated and optimized structures of the antibiotics were attached as .mol files.

\section{Referencess}

(1) Sheldrick, G. M. A Short History of SHELX. Acta Cryst A 2008, 64 (1), 112-122.

(2) Sheldrick, G. M. Crystal Structure Refinement with SHELXL. Acta Cryst C 2015, 71 (1), 3-8. 
(3) Dolomanov, O. V.; Bourhis, L. J.; Gildea, R. J.; Howard, J. a. K.; Puschmann, H. OLEX2: A Complete Structure Solution, Refinement and Analysis Program. J Appl Cryst 2009, 42 (2), 339-341.

(4) Pyta, K.; Przybylski, P.; Wicher, B.; Gdaniec, M.; Stefańska, J. Intramolecular Proton Transfer Impact on Antibacterial Properties of Ansamycin Antibiotic Rifampicin and Its New Amino Analogues. Org. Biomol. Chem. 2012, 10 (12), 2385-2388.

(5) Wicher, B.; Pyta, K.; Przybylski, P.; Tykarska, E.; Gdaniec, M. Redetermination of Rifampicin Pentahydrate Revealing a Zwitterionic Form of the Antibiotic. Acta Crystallographica Section C 2012, 68 (5), o209-o212.

(6) Campbell, E. A.; Korzheva, N.; Mustaev, A.; Murakami, K.; Nair, S.; Goldfarb, A.; Darst, S. A. Structural Mechanism for Rifampicin Inhibition of Bacterial RNA Polymerase. Cell 2001, 104 (6), 901-912.

\section{Figures}

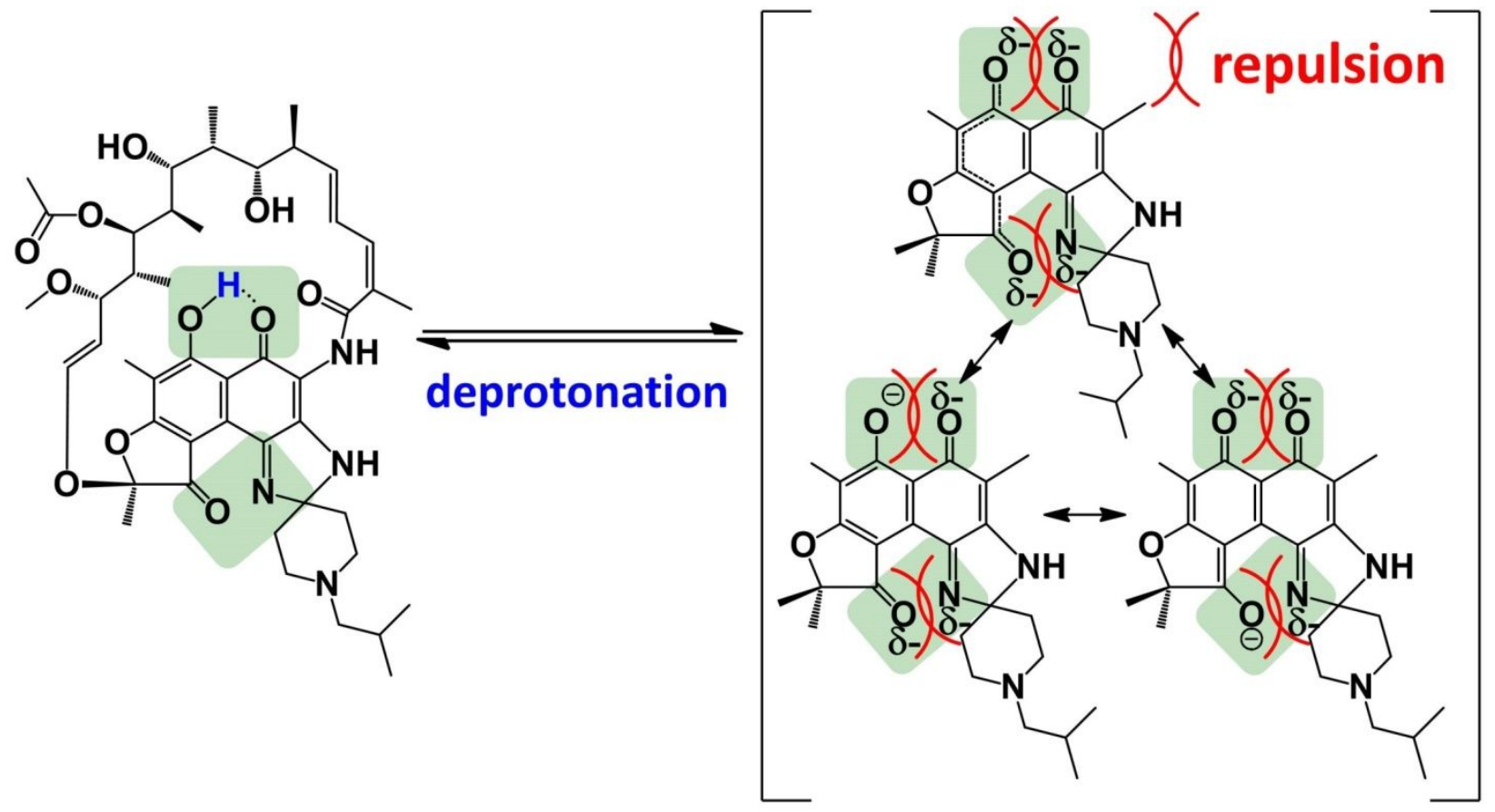

Fig. S1

Repulsion in RFB structure after (8)OH phenol group deprotonation. 

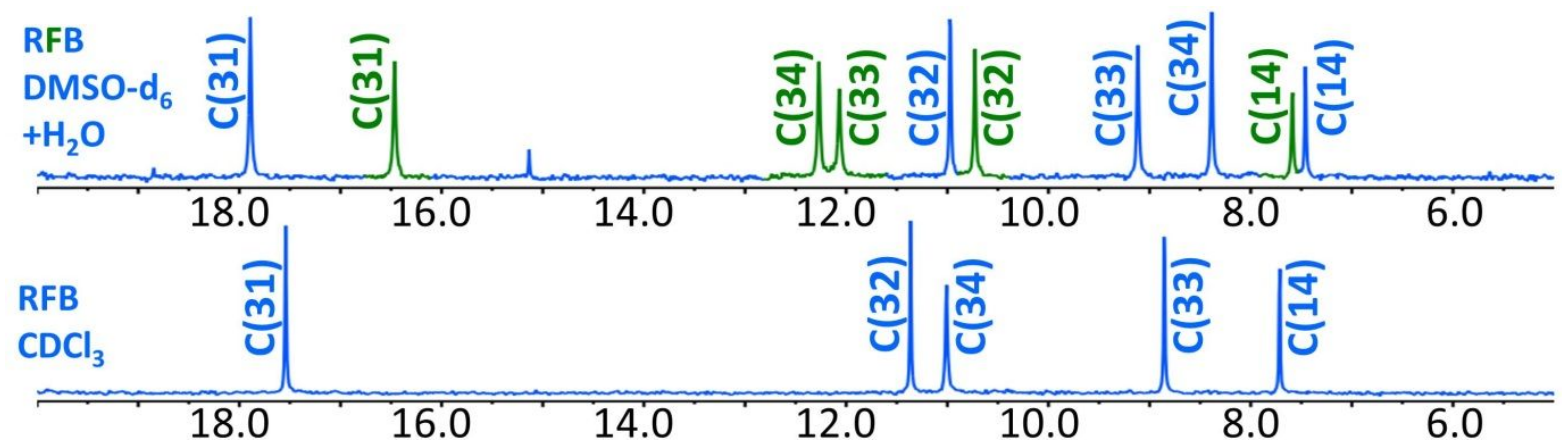

RFX

DMSO-d

$+\mathrm{H}_{2} \mathrm{O}$
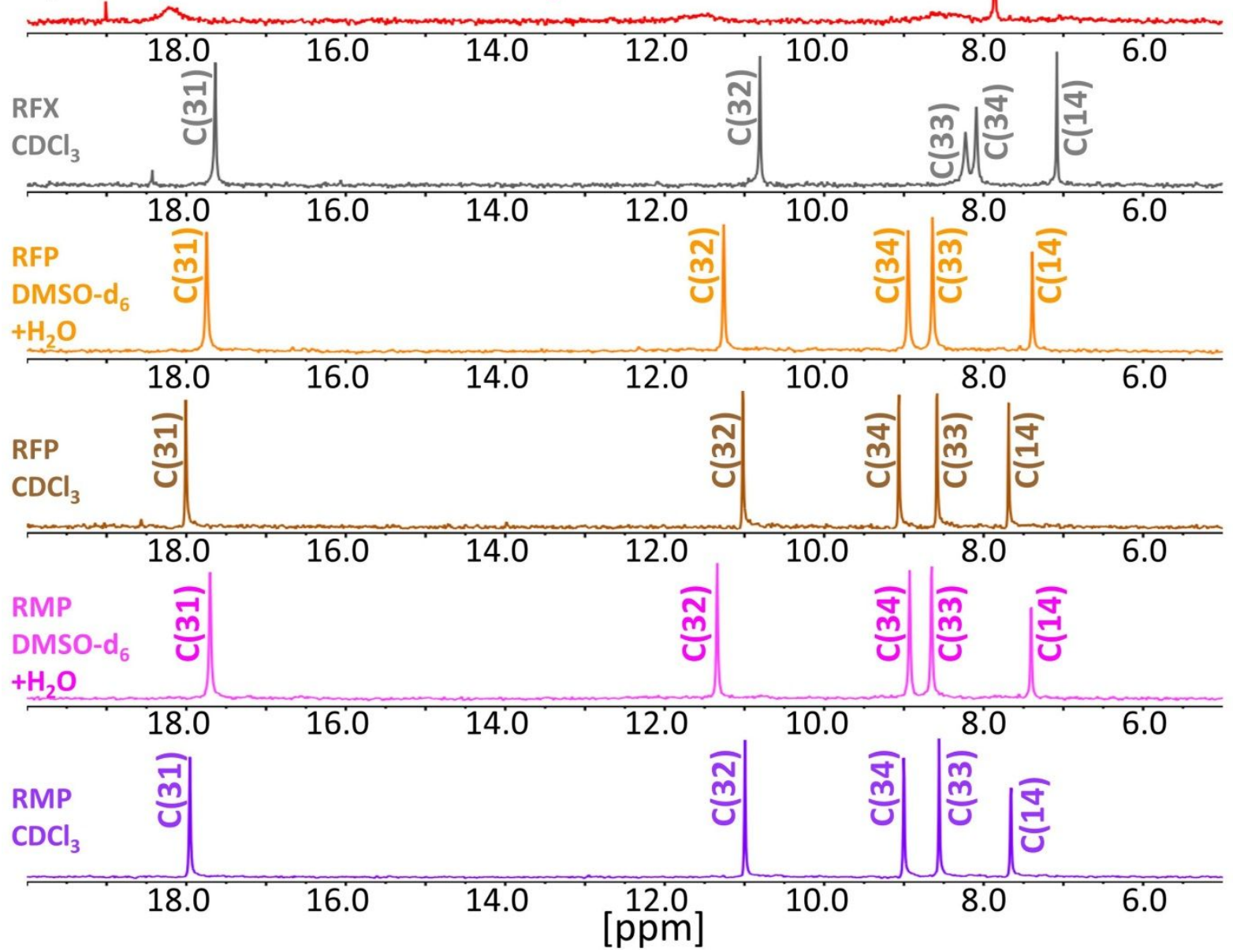

Fig. S2

Comparison of ${ }^{13} \mathrm{C}$ NMR spectra in the range 520 ppm for RMP, RFP, RFX and RFB measured in $\mathrm{CDCl}_{3}$ and DMSO- $\mathrm{d}_{6}+\mathrm{H}_{2} \mathrm{O}$ at room temperature, blue color was used to show signals for $\mathbf{R F B}(\mathbf{A})$ and green one for $\operatorname{RFB}(\mathbf{C})$, grey color was used to show signals for $\mathbf{R F X ( A )}$ and red one for $\mathbf{R F X ( B ) . ~}$ 

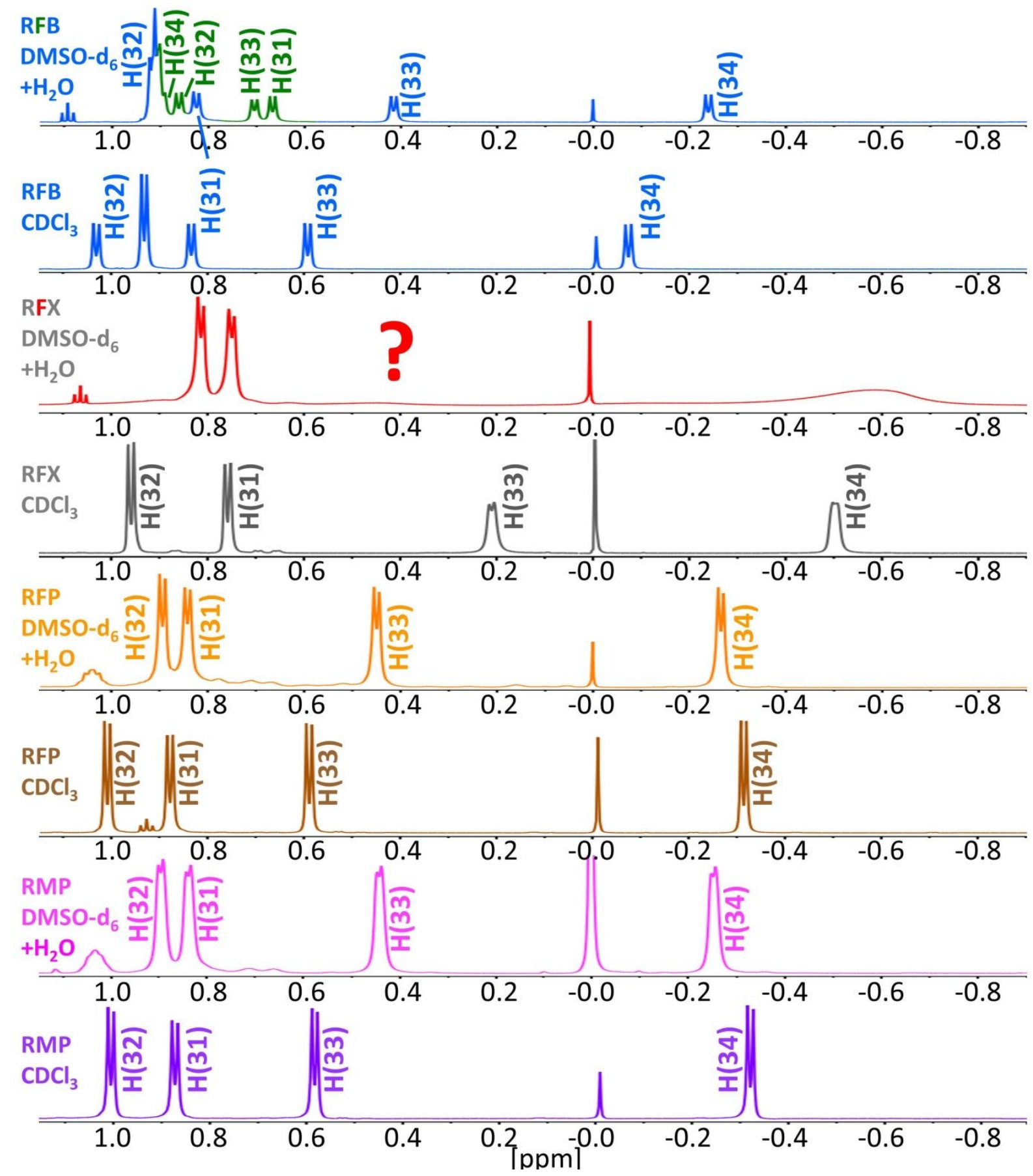

Fig. S3

Comparison of ${ }^{1} \mathrm{H}$ NMR spectra in the range -0.91 .15 ppm for RMP, RFP, RFX and RFB measured in $\mathrm{CDCl}_{3}$ and DMSO- $\mathrm{d}_{6}+\mathrm{H}_{2} \mathrm{O}$ at room temperature, blue color was used to show signals for $\mathbf{R F B}(\mathbf{A})$ and green one for $\mathbf{R F B}(\mathbf{C})$, grey color was used to show signals for RFX(A) and red one for $\mathbf{R F X ( B ) . ~}$ 

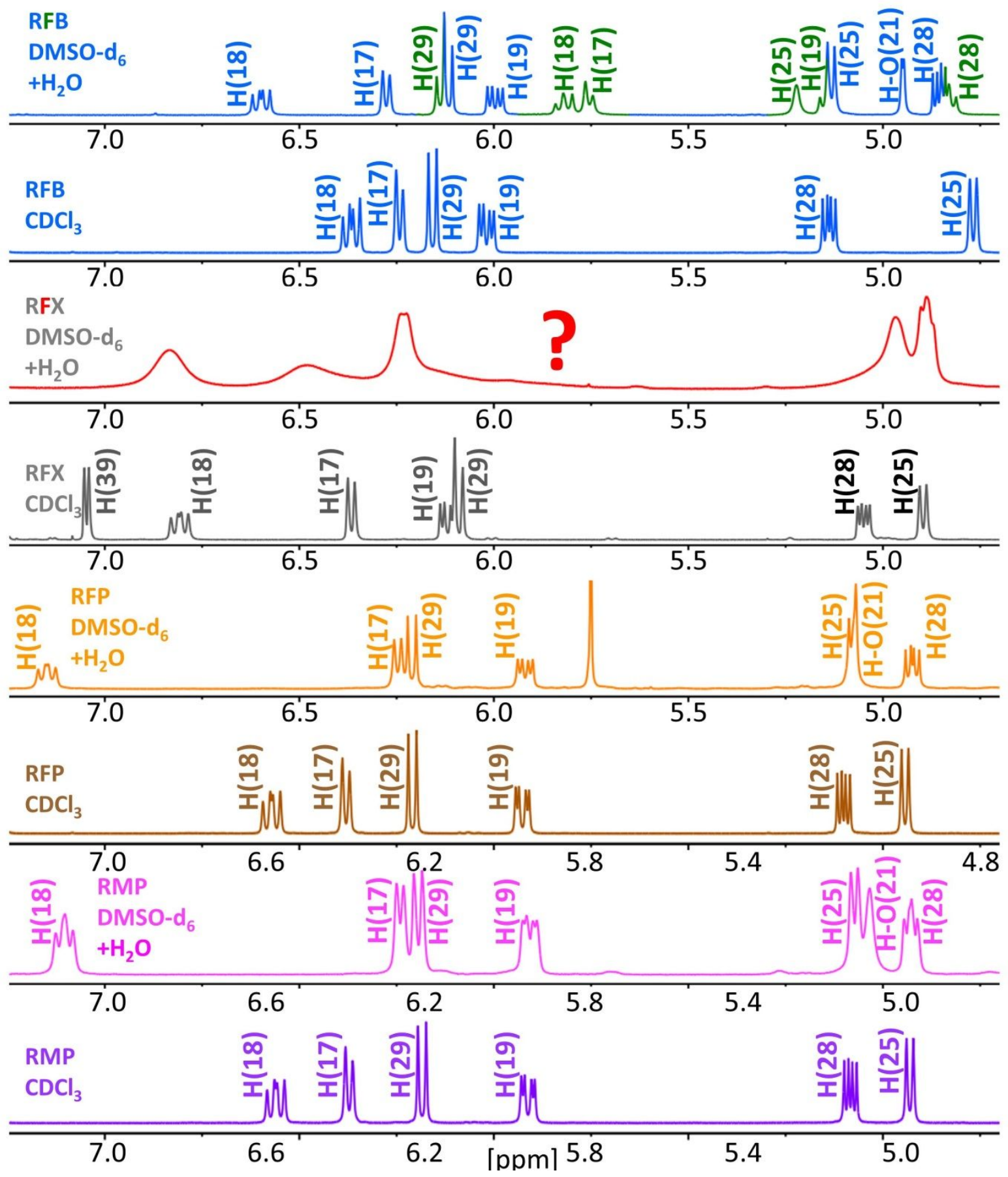

Fig. S4

Comparison of ${ }^{1} \mathrm{H}$ NMR spectra in the range $4.7 \quad 7.2$ ppm for RMP, RFP, RFX and RFB measured in $\mathrm{CDCl}_{3}$ and DMSO- $\mathrm{d}_{6}+\mathrm{H}_{2} \mathrm{O}$ at room temperature, blue color was used to show signals for $\mathbf{R F B}(\mathbf{A})$ and green one for $\operatorname{RFB}(\mathbf{C})$, grey color was used to show signals for $\operatorname{RFX(A)~and~red~one~for~} \mathbf{R F X ( B ) . ~}$ 

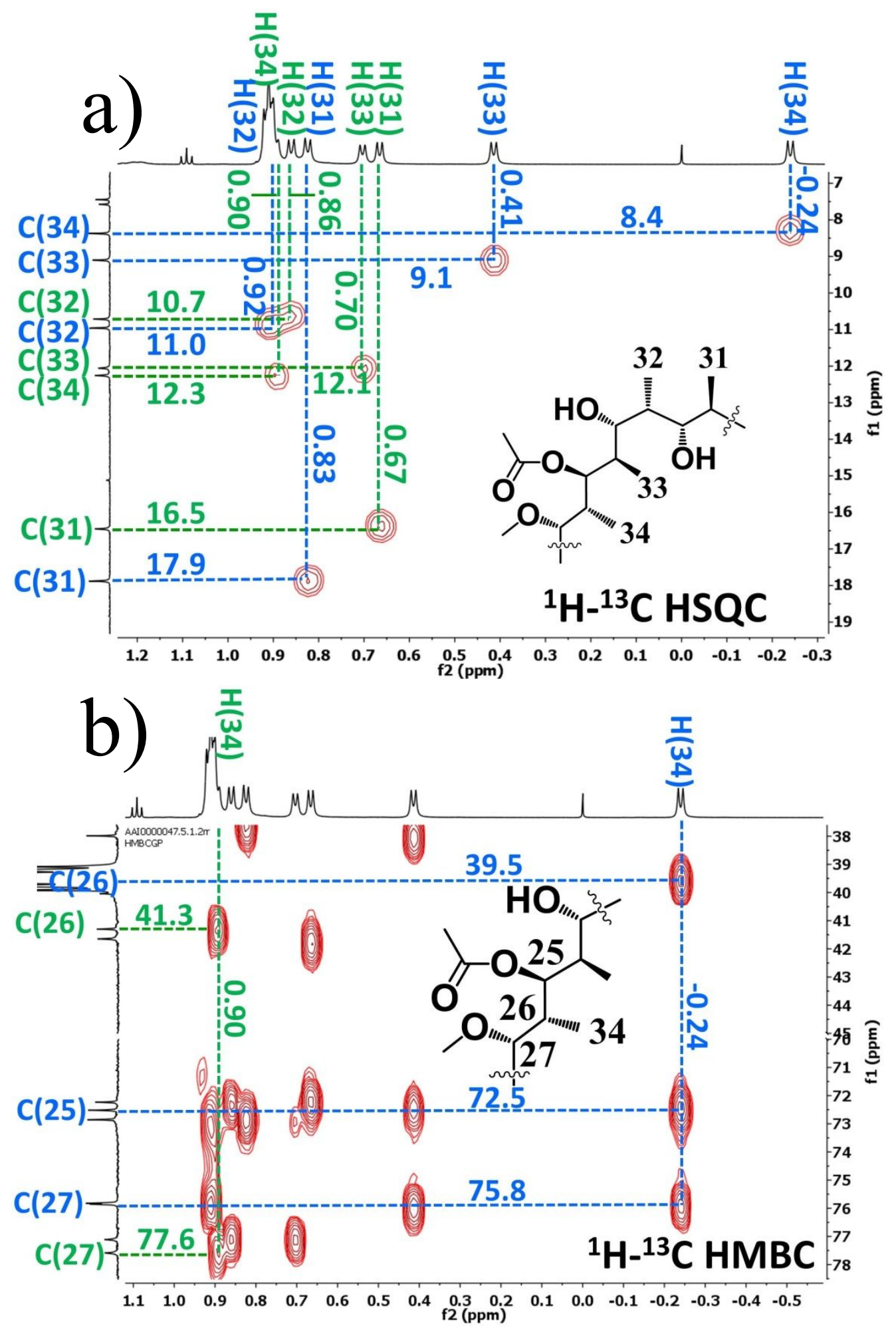

Fig. S5

a) fragment of ${ }^{1} \mathrm{H}_{-}{ }^{13} \mathrm{C}$ HSQC spectrum of RFB recorded in DMSO- $\mathrm{d}_{6}+\mathrm{H}_{2} \mathrm{O}$ at room temperatures; b) fragment of ${ }^{1} \mathrm{H}_{-}{ }^{13} \mathrm{C}$ HMBC spectrum of RFB recorded in DMSO- $\mathrm{d}_{6}+\mathrm{H}_{2} \mathrm{O}$ at room temperatures. Blue color was used to show signals for $\operatorname{RFB}(\mathbf{A})$ and green one for RFB(C). 

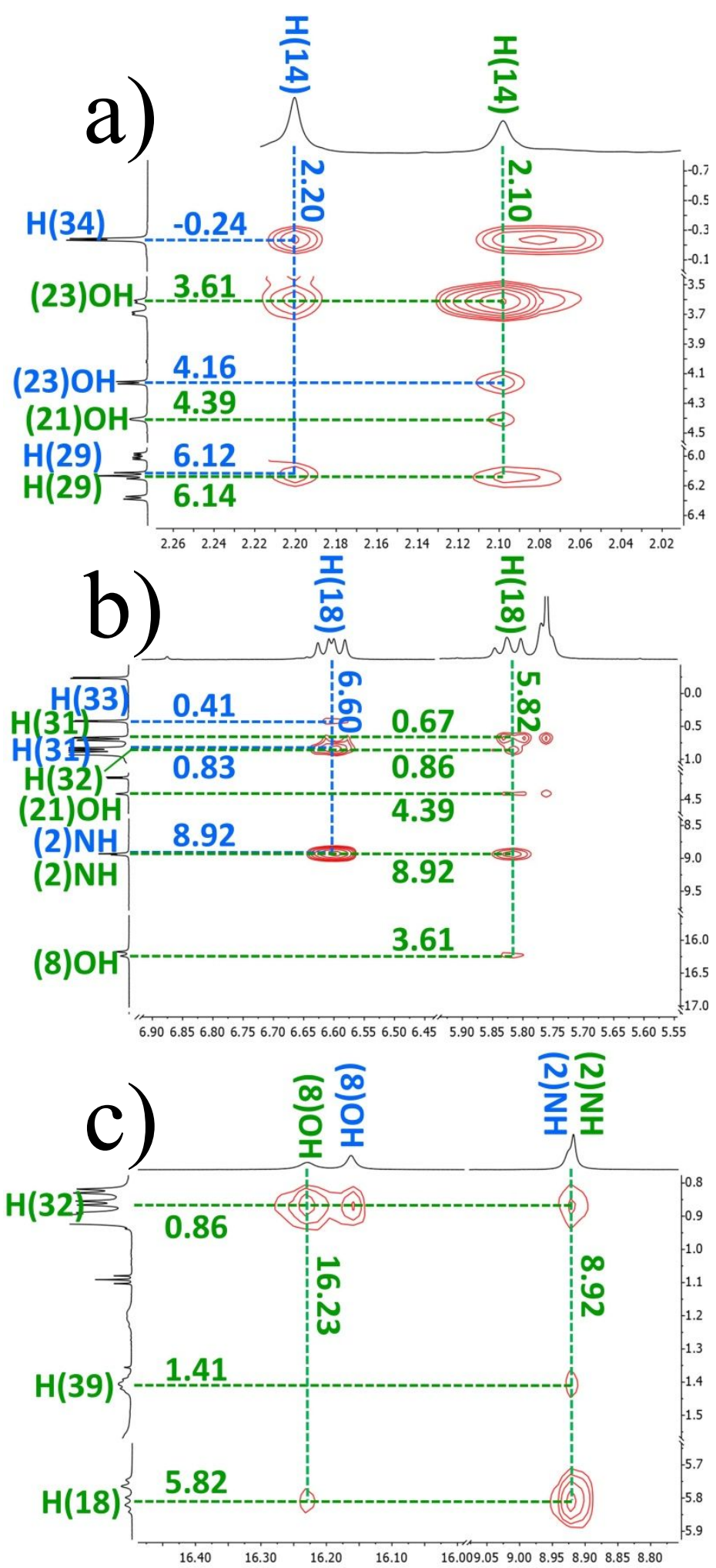

Fig. S6

Fragments of ${ }^{1} \mathrm{H}-{ }^{1} \mathrm{H}$ NOESY spectrum of RFB recorded in DMSO- $\mathrm{d}_{6}+\mathrm{H}_{2} \mathrm{O}$ at room temperatures: a) for $\mathrm{H}(14)$ signals, b) for $\mathrm{H}(18)$ signals and c) for (2)NH and (8)OH. Blue color was used to show signals for $\operatorname{RFB}(\mathbf{A})$ and green one for $\operatorname{RFB}(\mathbf{C})$. 

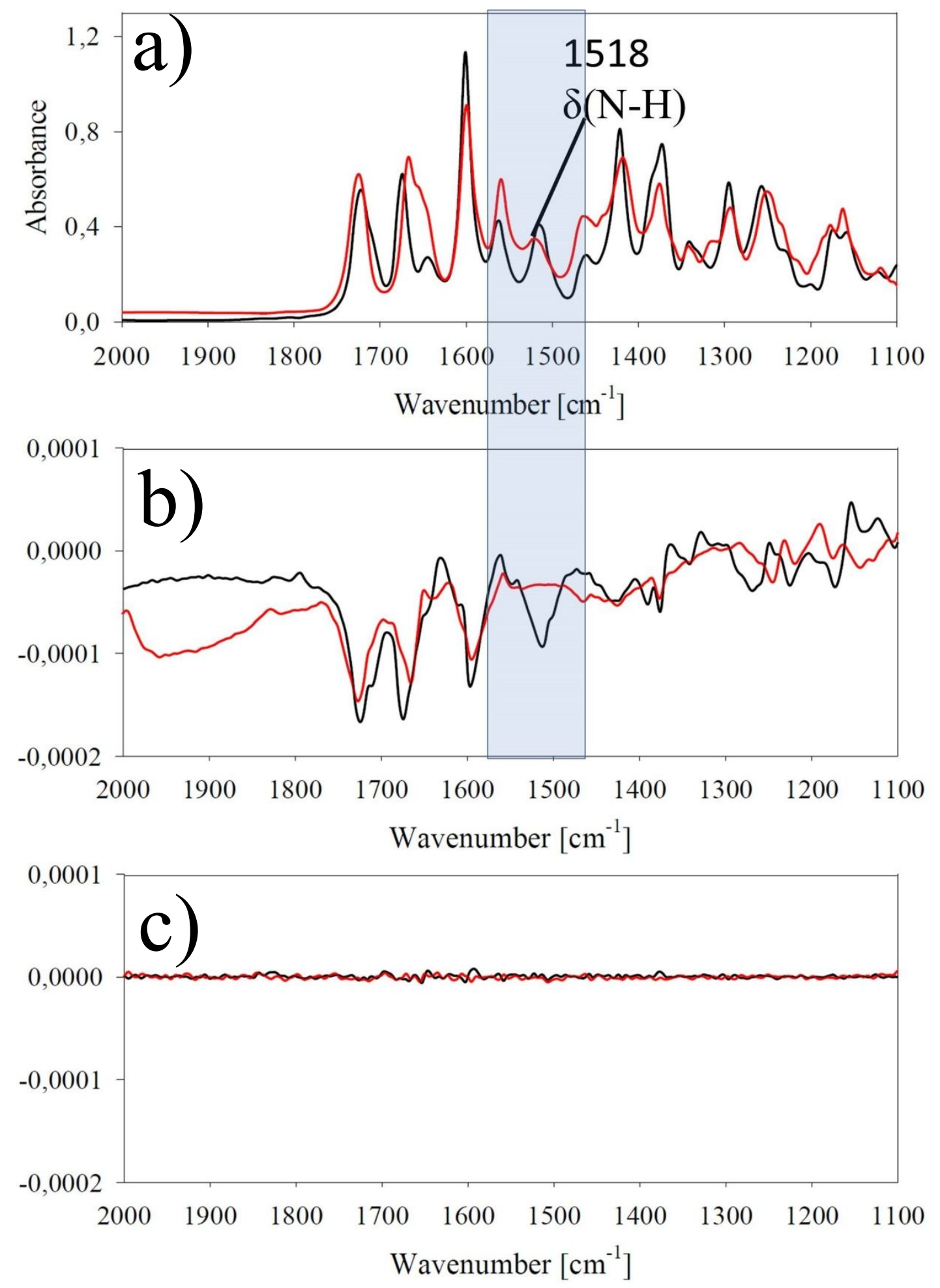

Fig. S7

Comparison of FT-IR spectra of RFB recorded in $\mathrm{CDCl}_{3}$ (solid black line) and in DMSO- $\mathrm{d}_{6}$ (red solid line), measured in 1100-2000 $\mathrm{cm}^{-1}$; b) comparison of VCD spectra of RFB recorded in $\mathrm{CDCl}_{3}$ (solid black line) and in DMSO- $\mathrm{d}_{6}$ (red solid line), measured in $1100-2000 \mathrm{~cm}^{-1}$; $\mathrm{c}$ ) comparison of noise spectra of RFB recorded in $\mathrm{CDCl}_{3}$ (solid black line) and in DMSO- $\mathrm{d}_{6}$ (red solid line), measured in $1100-2000 \mathrm{~cm}^{-1}$. 

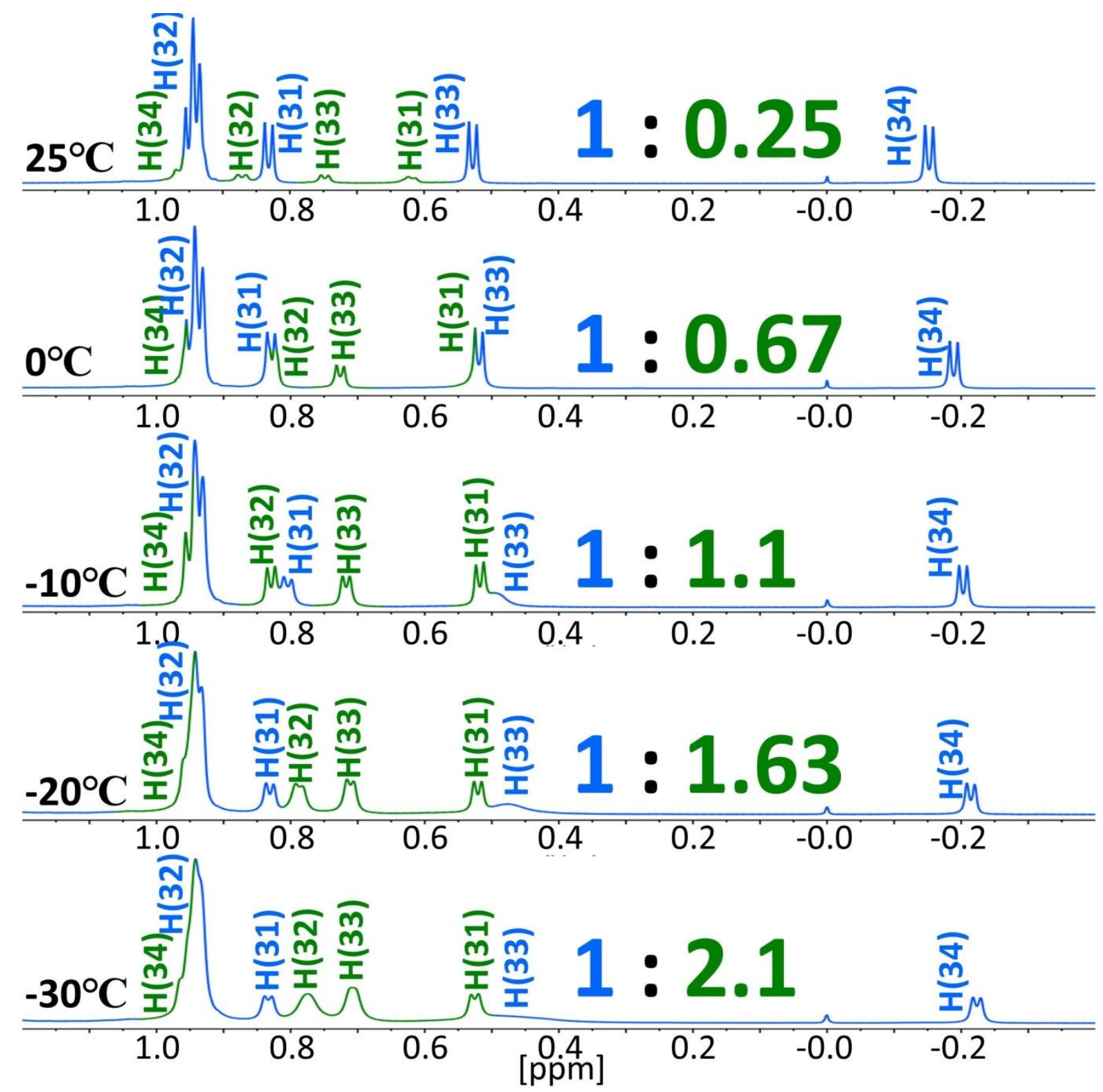

Fig. 58

Comparison of ${ }^{1} \mathrm{H}$ NMR spectra of RFB in the range $-0.4 \quad 1.2$ ppm measured in $\mathrm{CD}_{3} \mathrm{CN}+$ $\mathrm{H}_{2} \mathrm{O}$ at different temperatures, ratio of $\operatorname{RFB}(\mathbf{A}): \mathbf{R F B}(\mathbf{C})$ are shown above each spectrum, blue color was used to show signals for $\operatorname{RFB}(\mathbf{A})$ and green one for $\operatorname{RFB}(\mathbf{C})$. 


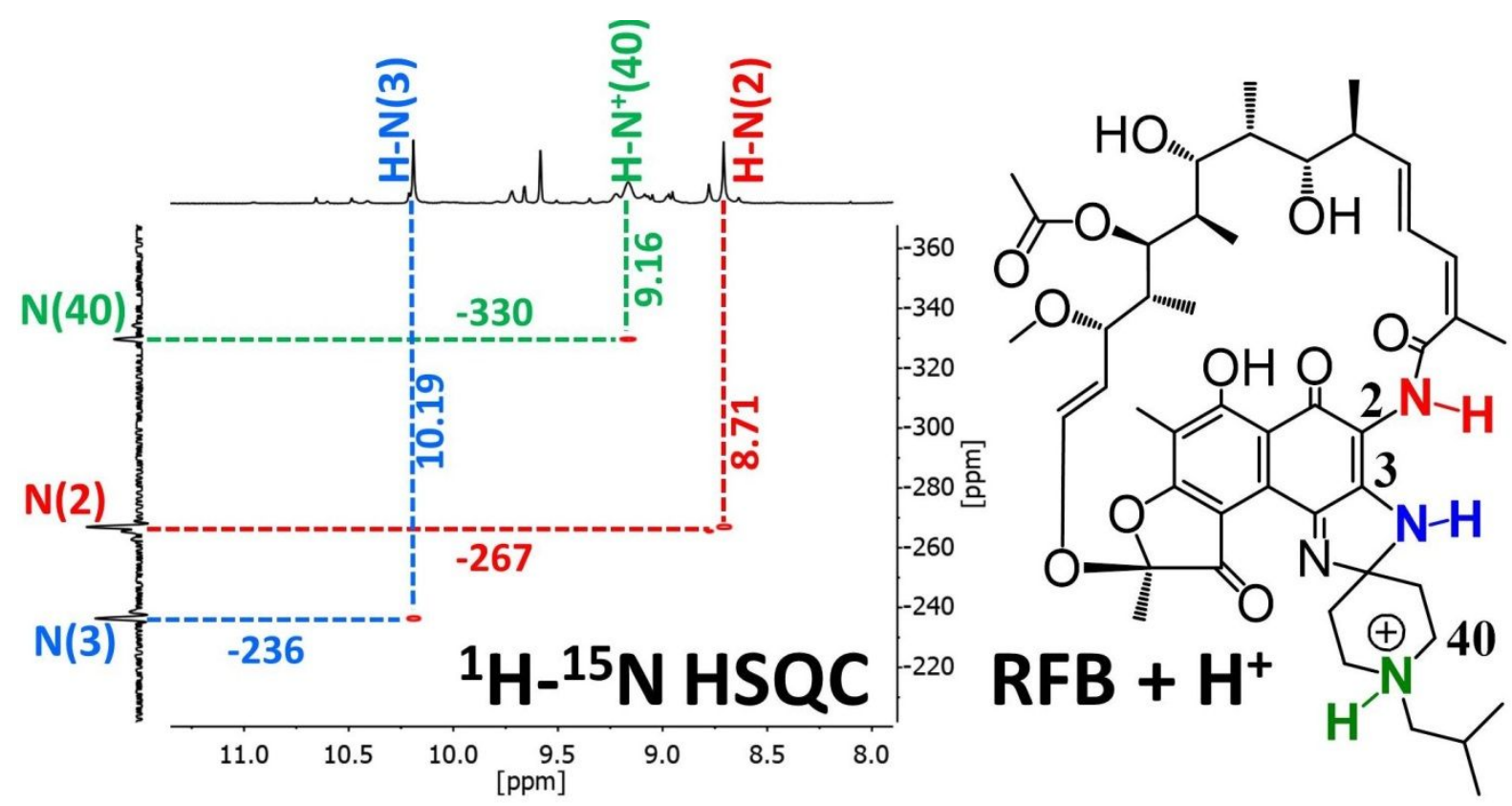

Fig. S9

Fragment of ${ }^{1} \mathrm{H}-{ }^{15} \mathrm{~N}$ HSQC spectrum of RFB after protonation recorded in DMSO- $\mathrm{d}_{6}+\mathrm{H}_{2} \mathrm{O}$ at room temperatures. 


\section{Tables}

\section{Table S1}

${ }^{1} \mathrm{H}$ NMR data of Rifampicin (RMP), Rifapentin (RFP) and Rifaximin (RFX) assigned on the basis of ${ }^{1} \mathrm{H}-{ }^{13} \mathrm{C}$ HSQC, ${ }^{1} \mathrm{H}-{ }^{13} \mathrm{C}$ HMBC, ${ }^{1} \mathrm{H}-{ }^{1} \mathrm{H}$ COSY and ${ }^{1} \mathrm{H}-{ }^{1} \mathrm{H}$ NOESY correlation spectra.

\begin{tabular}{|c|c|c|c|c|c|c|c|c|}
\hline $\begin{array}{l}\text { Proton } \\
\text { number }\end{array}$ & $\begin{array}{r}\mathbf{R M P} \\
\mathrm{CDCl}_{3}\end{array}$ & $\begin{array}{c}\text { RMP } \\
\text { DMSO-d }_{6}\end{array}$ & $\begin{array}{c}\mathbf{R F P} \\
\mathrm{CDCl}_{3}\end{array}$ & $\begin{array}{c}\text { RFP } \\
\text { DMSO-d }_{6}\end{array}$ & $\begin{array}{c}\mathbf{R F X}(\mathbf{A}) \\
\mathrm{CDCl}_{3}\end{array}$ & $\begin{array}{c}\text { RFX(A) } \\
\mathrm{CD}_{3} \mathrm{CN}: \\
\mathrm{DMSO}-\mathrm{d}_{6} \\
-30{ }^{\circ} \mathrm{C}\end{array}$ & $\begin{array}{c}\mathbf{R F X}(\mathbf{B}) \\
\mathrm{CD}_{3} \mathrm{CN}: \\
\mathrm{DMSO}-\mathrm{d}_{6} \\
-30{ }^{\circ} \mathrm{C}\end{array}$ & $\begin{array}{c}\text { RFX } \\
\mathrm{CD}_{3} \mathrm{CN} \\
25^{\circ} \mathrm{C}\end{array}$ \\
\hline 1 & -- & --- & --- & $\overline{---}$ & -- & --- & --- & --- \\
\hline 2 & -- & -- & -- & -- & -- & -- & -- & -- \\
\hline 3 & -- & -- & -- & -- & -- & -- & -- & -- \\
\hline 4 & -- & -- & -- & -- & -- & -- & -- & -- \\
\hline 5 & -- & -- & -- & -- & -- & --- & -- & --- \\
\hline 6 & -- & -- & -- & -- & -- & -- & -- & -- \\
\hline 7 & -- & -- & -- & -- & -- & -- & -- & -- \\
\hline 8 & -- & -- & -- & -- & -- & --- & -- & -- \\
\hline 9 & -- & -- & -- & -- & -- & -- & -- & --- \\
\hline 10 & -- & -- & -- & --- & -- & -- & -- & --- \\
\hline 11 & -- & -- & -- & -- & -- & -- & -- & -- \\
\hline 12 & -- & --- & -- & -- & -- & -- & -- & -- \\
\hline 13 & $\begin{array}{c}3 \mathrm{H} \\
1.81(\mathrm{~s})\end{array}$ & $\begin{array}{c}3 \mathrm{H} \\
1.64(\mathrm{~s})\end{array}$ & $\begin{array}{c}3 \mathrm{H} \\
1.80(\mathrm{~s})\end{array}$ & $\begin{array}{c}3 \mathrm{H} \\
1.63(\mathrm{~s})\end{array}$ & $\begin{array}{c}3 \mathrm{H} \\
1.95(\mathrm{~s})\end{array}$ & $\begin{array}{c}3 \mathrm{H} \\
1.91(\mathrm{~s})\end{array}$ & $\begin{array}{c}3 \mathrm{H} \\
1.86(\mathrm{~s})\end{array}$ & $\begin{array}{c}3 \mathrm{H} \\
1.88(\mathrm{~s})\end{array}$ \\
\hline 14 & $\begin{array}{c}3 \mathrm{H} \\
2.21(\mathrm{~s})\end{array}$ & $\begin{array}{c}3 \mathrm{H} \\
1.91(\mathrm{~s})\end{array}$ & $\begin{array}{c}3 \mathrm{H} \\
2.23(\mathrm{~s})\end{array}$ & $\begin{array}{c}3 \mathrm{H} \\
1.90(\mathrm{~s})\end{array}$ & $\begin{array}{c}3 \mathrm{H} \\
1.98(\mathrm{~s})\end{array}$ & $\begin{array}{c}3 \mathrm{H} \\
1.99(\mathrm{~s})\end{array}$ & $\begin{array}{c}3 \mathrm{H} \\
1.99(\mathrm{~s})\end{array}$ & $\begin{array}{c}3 \mathrm{H} \\
1.96(\mathrm{~s})\end{array}$ \\
\hline 15 & -- & - & -- & - & - & --- & -- & -- \\
\hline 16 & --- & --- & --- & --- & --- & --- & --- & -- \\
\hline
\end{tabular}




\begin{tabular}{|c|c|c|c|c|c|c|c|c|}
\hline $\begin{array}{l}\text { Proton } \\
\text { number }\end{array}$ & $\begin{array}{c}\mathbf{R M P} \\
\mathrm{CDCl}_{3}\end{array}$ & $\begin{array}{c}\text { RMP } \\
\text { DMSO-d }_{6}\end{array}$ & $\begin{array}{c}\mathbf{R F P} \\
\mathrm{CDCl}_{3}\end{array}$ & $\begin{array}{c}\text { RFP } \\
\text { DMSO-d }_{6}\end{array}$ & $\begin{array}{c}\mathbf{R F X ( A )} \\
\mathrm{CDCl}_{3}\end{array}$ & $\begin{array}{c}\text { RFX(A) } \\
\mathrm{CD}_{3} \mathrm{CN}: \\
\mathrm{DMSO} \mathrm{d}_{6} \\
-30^{\circ} \mathrm{C}\end{array}$ & $\begin{array}{c}\mathbf{R F X}(\mathbf{B}) \\
\mathrm{CD}_{3} \mathrm{CN}: \\
\mathrm{DMSO} \mathrm{d}_{6} \\
-30^{\circ} \mathrm{C}\end{array}$ & $\begin{array}{c}\text { RFX } \\
\mathrm{CD}_{3} \mathrm{CN} \\
25^{\circ} \mathrm{C}\end{array}$ \\
\hline 17 & $\begin{array}{c}1 \mathrm{H} \\
6.39(\mathrm{~d}) \\
{ }^{3} J_{\mathrm{H} 17-\mathrm{H} 18}=11.1\end{array}$ & $\begin{array}{c}1 \mathrm{H} \\
6.24(\mathrm{~d}) \\
{ }^{3} J_{\mathrm{H} 17-\mathrm{H} 18} \\
=10.8\end{array}$ & $\begin{array}{c}1 \mathrm{H} \\
6.39(\mathrm{~d}) \\
{ }^{3} J_{\mathrm{H} 17-\mathrm{H} 18}=11.2\end{array}$ & $\begin{array}{c}1 \mathrm{H} \\
6.24(\mathrm{~d}) \\
{ }^{3} J_{\mathrm{H} 17-\mathrm{H} 18} \\
=11.0 \\
\end{array}$ & $\begin{array}{c}1 \mathrm{H} \\
6.34(\mathrm{~d}) \\
{ }^{3} J_{\mathrm{H} 17-\mathrm{H} 18} \\
=10.6 \\
\end{array}$ & $\begin{array}{c}1 \mathrm{H} \\
6.31(\mathrm{~d}) \\
{ }^{3} J_{\mathrm{H} 17-\mathrm{H} 18} \\
=10.5 \\
\end{array}$ & $\begin{array}{c}1 \mathrm{H} \\
6.54(\mathrm{~d}) \\
{ }^{3} J_{\mathrm{H} 17-\mathrm{H} 18} \\
=9.7 \\
\end{array}$ & $\begin{array}{c}1 \mathrm{H} \\
6.40(\mathrm{~d}) \\
{ }^{3} J_{\mathrm{H} 17-\mathrm{H} 18} \\
=10.4 \\
\end{array}$ \\
\hline 18 & $\begin{array}{c}1 \mathrm{H} \\
6.58(\mathrm{dd}) \\
{ }^{3} J_{\mathrm{H} 18-\mathrm{H} 19}=15.2\end{array}$ & $\begin{array}{c}1 \mathrm{H} \\
7.09(\mathrm{dd}) \\
{ }^{3} J_{\mathrm{H} 18-\mathrm{H} 19} \\
=15.8\end{array}$ & $\begin{array}{c}1 \mathrm{H} \\
6.58(\mathrm{dd}) \\
{ }^{3} J_{\mathrm{H} 18-\mathrm{H} 19}=15.4\end{array}$ & $\begin{array}{c}1 \mathrm{H} \\
7.09(\mathrm{dd}) \\
{ }^{3} J_{\mathrm{H} 18-\mathrm{H} 19} \\
=16.0 \\
{ }^{3} J_{\mathrm{H} 17-\mathrm{H} 18} \\
=11.0\end{array}$ & $\begin{array}{c}1 \mathrm{H} \\
6.77(\mathrm{dd}) \\
{ }^{3} J_{\mathrm{H} 18-\mathrm{H} 19} \\
=16.0\end{array}$ & $\begin{array}{c}1 \mathrm{H} \\
5.67(\mathrm{~m})\end{array}$ & $\begin{array}{c}1 \mathrm{H} \\
6.82(\mathrm{~m})\end{array}$ & $\begin{array}{c}1 \mathrm{H} \\
6.78(\mathrm{dd}) \\
{ }^{3} J_{\mathrm{H} 18-\mathrm{H} 19} \\
=16.1\end{array}$ \\
\hline 19 & $\begin{array}{c}1 \mathrm{H} \\
5.93(\mathrm{dd}) \\
{ }^{3} J_{\mathrm{H} 19-\mathrm{H} 20}=4.9\end{array}$ & $\begin{array}{c}1 \mathrm{H} \\
5.91(\mathrm{dd}) \\
{ }^{3} J_{\mathrm{H} 19-\mathrm{H} 20}=7.1\end{array}$ & $\begin{array}{c}1 \mathrm{H} \\
5.93(\mathrm{dd}) \\
{ }^{3} J_{\mathrm{H} 19-\mathrm{H} 20}=5.0\end{array}$ & $\begin{array}{c}1 \mathrm{H} \\
5.92(\mathrm{dd}) \\
{ }^{3} J_{\mathrm{H} 18-\mathrm{H} 19} \\
=16.0 \\
{ }^{3} J_{\mathrm{H} 19-\mathrm{H} 20}=7.0\end{array}$ & $\begin{array}{c}1 \mathrm{H} \\
6.07(\mathrm{dd}) \\
{ }^{{ }^{3} J_{\mathrm{H} 19-\mathrm{H} 20}=6.6}\end{array}$ & $\begin{array}{c}1 \mathrm{H} \\
5.88(\mathrm{dd}) \\
{ }^{3} J_{\mathrm{H} 19-\mathrm{H} 20}=5.9 \\
{ }^{3} J_{\mathrm{H} 18-\mathrm{H} 19} \\
=15.9\end{array}$ & $\begin{array}{c}1 \mathrm{H} \\
6.19(\mathrm{~m})\end{array}$ & $\begin{array}{c}1 \mathrm{H} \\
6.00(\mathrm{dd}) \\
{ }^{3} J_{\mathrm{H} 19-\mathrm{H} 20}=6.7\end{array}$ \\
\hline 20 & $\begin{array}{c}1 \mathrm{H} \\
2.38(\mathrm{~m})\end{array}$ & $\begin{array}{c}1 \mathrm{H} \\
2.19(\mathrm{~m})\end{array}$ & $\begin{array}{c}1 \mathrm{H} \\
2.38(\mathrm{~m})\end{array}$ & $\begin{array}{c}1 \mathrm{H} \\
2.19(\mathrm{~m})\end{array}$ & $\begin{array}{c}1 \mathrm{H} \\
2.29(\mathrm{~m})\end{array}$ & $\begin{array}{c}1 \mathrm{H} \\
2.10(\mathrm{~m})\end{array}$ & $\begin{array}{c}1 \mathrm{H} \\
2.24(\mathrm{~m})\end{array}$ & $\begin{array}{c}1 \mathrm{H} \\
2.21(\mathrm{~m})\end{array}$ \\
\hline 21 & & $\begin{array}{c}1 \mathrm{H} \\
3.76(\mathrm{~d}) \\
{ }^{3} J_{\mathrm{H} 20-\mathrm{H} 21}=9.0\end{array}$ & $\begin{array}{c}1 \mathrm{H} \\
3.78(\mathrm{~d}) \\
{ }_{3} J_{\mathrm{H} 20-\mathrm{H} 21}=9.5\end{array}$ & $\begin{array}{c}1 \mathrm{H} \\
3.77(\mathrm{~d}) \\
{ }^{3} J_{\mathrm{H} 20-\mathrm{H} 21}=9.1\end{array}$ & $\begin{array}{c}1 \mathrm{H} \\
3.58(\mathrm{~d}) \\
{ }^{3} J_{\mathrm{H} 20-\mathrm{H} 21}=9.7\end{array}$ & $\begin{array}{c}1 \mathrm{H} \\
3.48(\mathrm{~d}) \\
{ }^{3} \mathrm{~J}_{\mathrm{H} 20-\mathrm{H} 21}=9.3\end{array}$ & $\begin{array}{c}1 \mathrm{H} \\
3.41(\mathrm{~m})\end{array}$ & $\begin{array}{c}1 \mathrm{H} \\
3.49(\mathrm{dd}) \\
{ }^{3} J_{\mathrm{H} 21-\mathrm{H} 22}=2.2 \\
{ }^{3} J_{\mathrm{H} 20 \mathrm{-} 21}=9.2\end{array}$ \\
\hline 22 & $\begin{array}{c}1 \mathrm{H} \\
1.71(\mathrm{~m})\end{array}$ & $\begin{array}{c}1 \mathrm{H} \\
1.59(\mathrm{~m})\end{array}$ & $\begin{array}{c}1 \mathrm{H} \\
1.71(\mathrm{~m})\end{array}$ & $\begin{array}{c}1 \mathrm{H} \\
1.59(\mathrm{~m})\end{array}$ & $\begin{array}{c}1 \mathrm{H} \\
1.56(\mathrm{~m})\end{array}$ & $\begin{array}{c}1 \mathrm{H} \\
1.07(\mathrm{~m})\end{array}$ & $\begin{array}{c}1 \mathrm{H} \\
1.33(\mathrm{~m})\end{array}$ & $\begin{array}{c}1 \mathrm{H} \\
1.52(\mathrm{~m})\end{array}$ \\
\hline 23 & $\begin{array}{c}1 \mathrm{H} \\
3.02(\mathrm{~m})^{*}\end{array}$ & $\begin{array}{c}1 \mathrm{H} \\
2.83(\mathrm{~m})\end{array}$ & $\begin{array}{c}1 \mathrm{H} \\
3.02 \\
(\mathrm{~m})^{*}\end{array}$ & $\begin{array}{c}1 \mathrm{H} \\
2.81(\mathrm{~m})\end{array}$ & $\begin{array}{c}1 \mathrm{H} \\
2.85(\mathrm{~m})\end{array}$ & $\begin{array}{c}1 \mathrm{H} \\
2.77(\mathrm{~m})\end{array}$ & $\begin{array}{c}1 \mathrm{H} \\
2.56(\mathrm{~m})\end{array}$ & $\begin{array}{c}1 \mathrm{H} \\
2.80(\mathrm{~m})\end{array}$ \\
\hline 24 & $\begin{array}{c}1 \mathrm{H} \\
1.53(\mathrm{qd}) \\
{ }^{3} J_{\mathrm{H} 24-\mathrm{H} 33}=6.8 \\
{ }^{3} J_{\mathrm{H} 23-\mathrm{H} 24}=14.0\end{array}$ & $\begin{array}{c}1 \mathrm{H} \\
1.33(\mathrm{~m})\end{array}$ & $\begin{array}{c}1 \mathrm{H} \\
1.54(\mathrm{~m})\end{array}$ & $\begin{array}{c}1 \mathrm{H} \\
1.31(\mathrm{~m})\end{array}$ & $\begin{array}{c}1 \mathrm{H} \\
1.25(\mathrm{~m})\end{array}$ & $\begin{array}{c}1 \mathrm{H} \\
1.09(\mathrm{~m})\end{array}$ & $\begin{array}{c}1 \mathrm{H} \\
0.76(\mathrm{~m})\end{array}$ & $\begin{array}{c}1 \mathrm{H} \\
1.10(\mathrm{~m})\end{array}$ \\
\hline
\end{tabular}




\begin{tabular}{|c|c|c|c|c|c|c|c|c|}
\hline $\begin{array}{l}\text { Proton } \\
\text { number }\end{array}$ & $\begin{array}{c}\mathbf{R M P} \\
\mathrm{CDCl}_{3}\end{array}$ & $\begin{array}{c}\text { RMP } \\
\text { DMSO-d }_{6}\end{array}$ & $\begin{array}{c}\mathbf{R F P} \\
\mathrm{CDCl}_{3}\end{array}$ & $\begin{array}{c}\text { RFP } \\
\text { DMSO-d }_{6}\end{array}$ & $\begin{array}{c}\mathbf{R F X}(\mathbf{A}) \\
\mathrm{CDCl}_{3}\end{array}$ & $\begin{array}{c}\text { RFX(A) } \\
\mathrm{CD}_{3} \mathrm{CN}: \\
\mathrm{DMSO} \mathrm{d}_{6} \\
-30^{\circ} \mathrm{C}\end{array}$ & $\begin{array}{c}\text { RFX(B) } \\
\mathrm{CD}_{3} \mathrm{CN}: \\
\mathrm{DMSO} \mathrm{d}_{6} \\
-30^{\circ} \mathrm{C}\end{array}$ & $\begin{array}{c}\text { RFX } \\
\mathrm{CD}_{3} \mathrm{CN} \\
25^{\circ} \mathrm{C}\end{array}$ \\
\hline 25 & $\begin{array}{c}1 \mathrm{H} \\
4.95(\mathrm{~d}) \\
{ }^{3} J_{\mathrm{H} 25-\mathrm{H} 26}=10.6\end{array}$ & $\begin{array}{c}1 \mathrm{H} \\
5.06(\mathrm{~d}) \\
{ }^{3} J_{\mathrm{H} 25-\mathrm{H} 26} \\
=10.9 \\
\end{array}$ & $\begin{array}{c}1 \mathrm{H} \\
4.95(\mathrm{~d}) \\
{ }^{3} J_{\mathrm{H} 25-\mathrm{H} 26}=10.6\end{array}$ & $\begin{array}{c}1 \mathrm{H} \\
5.07(\mathrm{~d}) \\
{ }^{3} \mathrm{~J}_{\mathrm{H} 25-\mathrm{H} 26} \\
=10.7 \\
\end{array}$ & $\begin{array}{c}1 \mathrm{H} \\
4.85(\mathrm{~d}) \\
{ }^{3} J_{\mathrm{H} 25-\mathrm{H} 26}=10.5\end{array}$ & $\begin{array}{c}1 \mathrm{H} \\
5.04(\mathrm{~d}) \\
{ }^{3} J_{\mathrm{H} 25-\mathrm{H} 26} \\
=10.5 \\
\end{array}$ & $\begin{array}{c}1 \mathrm{H} \\
4.86(\mathrm{~d}) \\
{ }^{3} J_{\mathrm{H} 25-\mathrm{H} 26}=11.3\end{array}$ & $\begin{array}{c}1 \mathrm{H} \\
4.86(\mathrm{~d}) \\
{ }^{3} J_{\mathrm{H} 25-\mathrm{H} 26}=10.6\end{array}$ \\
\hline 26 & $\begin{array}{c}1 \mathrm{H} \\
1.35(\mathrm{~m})\end{array}$ & $\begin{array}{c}1 \mathrm{H} \\
1.01(\mathrm{~m})\end{array}$ & $\begin{array}{c}1 \mathrm{H} \\
1.35(\mathrm{~m})\end{array}$ & $\begin{array}{c}1 \mathrm{H} \\
1.03(\mathrm{~m})\end{array}$ & $\begin{array}{c}1 \mathrm{H} \\
1.08(\mathrm{~m})\end{array}$ & $\begin{array}{c}1 \mathrm{H} \\
0.88(\mathrm{~m})\end{array}$ & $\begin{array}{c}1 \mathrm{H} \\
0.25(\mathrm{~m})\end{array}$ & $\begin{array}{c}1 \mathrm{H} \\
0.92(\mathrm{~m})\end{array}$ \\
\hline 27 & $\begin{array}{c}1 \mathrm{H} \\
3.51(\mathrm{~d}) \\
{ }^{3} J_{\mathrm{H} 27-\mathrm{H} 28}=6.7\end{array}$ & $\begin{array}{c}1 \mathrm{H} \\
3.23(\mathrm{~d}) \\
J_{\mathrm{H} 27-\mathrm{H} 28}=8.3\end{array}$ & $\begin{array}{c}1 \mathrm{H} \\
3.47(\mathrm{~m})\end{array}$ & $\begin{array}{c}1 \mathrm{H} \\
3.23(\mathrm{~d}) \\
{ }_{3} J_{\mathrm{H} 27-\mathrm{H} 28}=8.3\end{array}$ & $\begin{array}{c}1 \mathrm{H} \\
3.27(\mathrm{dd}) \\
{ }^{3} J_{\mathrm{H} 27-\mathrm{H} 28=6.8}=6 \\
{ }^{3} J_{\mathrm{H} 26-\mathrm{H} 27}=1.1\end{array}$ & $\begin{array}{c}1 \mathrm{H} \\
3.20(\mathrm{~d}) \\
{ }_{3} J_{\mathrm{H} 27-\mathrm{H} 28}=6.1\end{array}$ & $\begin{array}{c}1 \mathrm{H} \\
2.99(\mathrm{~m})\end{array}$ & $\begin{array}{c}1 \mathrm{H} \\
3.25(\mathrm{~d}) \\
{ }^{3} J_{\mathrm{H} 27-\mathrm{H} 28}=6.6\end{array}$ \\
\hline 28 & $\begin{array}{c}1 \mathrm{H} \\
5.10(\mathrm{dd}) \\
{ }^{3} J_{\mathrm{H} 28-\mathrm{H} 29}=12.6\end{array}$ & $\begin{array}{c}1 \mathrm{H} \\
4.93 \\
(\mathrm{dd}) \\
{ }^{3} \mathrm{~J}_{\mathrm{H} 28-\mathrm{H} 29} \\
=12.8\end{array}$ & $\begin{array}{c}1 \mathrm{H} \\
5.11(\mathrm{dd}) \\
{ }^{3} J_{\mathrm{H} 28-\mathrm{H} 29}=12.7 \\
{ }^{3} J_{\mathrm{H} 27-\mathrm{H} 28}=6.8\end{array}$ & $\begin{array}{c}1 \mathrm{H} \\
4.92 \\
(\mathrm{dd}) \\
{ }^{3} J_{\mathrm{H} 28-\mathrm{H} 29} \\
=12.8 \\
{ }^{3} J_{\mathrm{H} 27-\mathrm{H} 28}=8.3\end{array}$ & $\begin{array}{c}1 \mathrm{H} \\
4.99 \\
(\mathrm{dd}) \\
{ }^{3} J_{\mathrm{H} 28-\mathrm{H} 29} \\
=12.1 \\
{ }^{3} J_{\mathrm{H} 27-\mathrm{H} 28}=5.9\end{array}$ & $\begin{array}{c}1 \mathrm{H} \\
4.81 \\
(\mathrm{~m})\end{array}$ & $\begin{array}{c}1 \mathrm{H} \\
4.71 \\
(\mathrm{~m})\end{array}$ & $\begin{array}{c}1 \mathrm{H} \\
4.91 \\
(\mathrm{dd}) \\
J_{\mathrm{H} 28-\mathrm{H} 27} \\
=6.6 \\
3 J_{\mathrm{H} 28-\mathrm{H} 29} \\
=12.6 \\
\end{array}$ \\
\hline 29 & $\begin{array}{c}1 \mathrm{H} \\
6.20(\mathrm{~d})\end{array}$ & $\begin{array}{c}1 \mathrm{H} \\
6.20(\mathrm{~d})\end{array}$ & $\begin{array}{c}1 \mathrm{H} \\
6.22(\mathrm{~d})\end{array}$ & $\begin{array}{c}1 \mathrm{H} \\
6.21(\mathrm{~d}) \\
{ }^{3} J_{\mathrm{H} 28-\mathrm{H} 29} \\
=122.8\end{array}$ & $\begin{array}{c}1 \mathrm{H} \\
6.03(\mathrm{~d})\end{array}$ & $\begin{array}{c}1 \mathrm{H} \\
6.08(\mathrm{~d})^{3} J_{\mathrm{H} 28-} \\
=129 \\
=12.5\end{array}$ & $\begin{array}{c}1 \mathrm{H} \\
6.21(\mathrm{~d})^{3} J_{\mathrm{H} 28-} \\
=11.3\end{array}$ & $\begin{array}{c}1 \mathrm{H} \\
6.05(\mathrm{~d})\end{array}$ \\
\hline 30 & $\begin{array}{c}3 \mathrm{H} \\
2.09(\mathrm{~s})\end{array}$ & $\begin{array}{c}3 \mathrm{H} \\
1.91(\mathrm{~s})\end{array}$ & $\begin{array}{c}3 \mathrm{H} \\
2.08(\mathrm{~s})\end{array}$ & $\begin{array}{c}3 \mathrm{H} \\
1.90(\mathrm{~s})\end{array}$ & $\begin{array}{c}3 \mathrm{H} \\
2.24(\mathrm{~s})\end{array}$ & $\begin{array}{c}3 \mathrm{H} \\
2.26(\mathrm{~s})\end{array}$ & $\begin{array}{c}3 \mathrm{H} \\
2.13(\mathrm{~s})\end{array}$ & $\begin{array}{c}3 \mathrm{H} \\
2.23(\mathrm{~s})\end{array}$ \\
\hline 31 & $\begin{array}{c}3 \mathrm{H} \\
0.88(\mathrm{~d}) \\
{ }^{3} J_{\mathrm{H} 20-\mathrm{H} 31}=7.0\end{array}$ & $\begin{array}{c}3 \mathrm{H} \\
0.83(\mathrm{~d}) \\
{ }^{3} J_{\mathrm{H} 20-\mathrm{H} 31}=6.9\end{array}$ & $\begin{array}{c}3 \mathrm{H} \\
0.89(\mathrm{~d}) \\
{ }^{3} J_{\mathrm{H} 20-\mathrm{H} 31}=7.1\end{array}$ & $\begin{array}{c}3 \mathrm{H} \\
0.84(\mathrm{~d}) \\
{ }^{3} J_{\mathrm{H} 20-\mathrm{H} 31}=6.9\end{array}$ & $\begin{array}{c}3 \mathrm{H} \\
0.73(\mathrm{~d}) \\
{ }^{3} J_{\mathrm{H} 20-\mathrm{H} 31}=7.1\end{array}$ & $\begin{array}{c}3 \mathrm{H} \\
0.63(\mathrm{~d}) \\
{ }^{3} J_{\mathrm{H} 20-\mathrm{H} 31}=6.9\end{array}$ & $\begin{array}{c}3 \mathrm{H} \\
0.73(\mathrm{~d}) \\
{ }^{3} J_{\mathrm{H} 20-\mathrm{H} 31}=6.9\end{array}$ & $\begin{array}{c}3 \mathrm{H} \\
0.73 \text { (d) } \\
{ }^{3} J_{\mathrm{H} 20-\mathrm{H} 31}=6.9\end{array}$ \\
\hline 32 & $\begin{array}{c}3 \mathrm{H} \\
1.01(\mathrm{~d}) \\
{ }_{3} J_{\mathrm{H} 22-\mathrm{H} 32}=7.0\end{array}$ & $\begin{array}{c}3 \mathrm{H} \\
0.89(\mathrm{~d}) \\
3 J_{\mathrm{H} 22-\mathrm{H} 2}=7.1\end{array}$ & $\begin{array}{c}3 \mathrm{H} \\
1.02(\mathrm{~d}) \\
{ }_{3} J_{\mathrm{H} 22-\mathrm{H} 2}=6.9\end{array}$ & $\begin{array}{c}3 \mathrm{H} \\
0.89(\mathrm{~d}) \\
3 J_{\mathrm{H} 22-\mathrm{H} 22}=6.9\end{array}$ & $\begin{array}{c}3 \mathrm{H} \\
0.92(\mathrm{~d}) \\
{ }_{3} J_{\mathrm{H} 22-\mathrm{H} 32}=7.1\end{array}$ & $\begin{array}{c}3 \mathrm{H} \\
0.79(\mathrm{~m})\end{array}$ & $\begin{array}{c}3 \mathrm{H} \\
0.79(\mathrm{~m})\end{array}$ & $\begin{array}{c}3 \mathrm{H} \\
0.86(\mathrm{~d}) \\
{ }^{3} J_{\mathrm{H} 22-\mathrm{H} 32}=7.1\end{array}$ \\
\hline
\end{tabular}




\begin{tabular}{|c|c|c|c|c|c|c|c|c|}
\hline $\begin{array}{l}\text { Proton } \\
\text { number }\end{array}$ & $\begin{array}{c}\mathbf{R M P} \\
\mathrm{CDCl}_{3}\end{array}$ & $\begin{array}{c}\text { RMP } \\
\text { DMSO- }_{6}\end{array}$ & $\begin{array}{c}\mathbf{R F P} \\
\mathrm{CDCl}_{3}\end{array}$ & $\begin{array}{c}\text { RFP } \\
\text { DMSO-d }_{6}\end{array}$ & $\begin{array}{c}\mathbf{R F X ( A )} \\
\mathrm{CDCl}_{3}\end{array}$ & $\begin{array}{c}\text { RFX(A) } \\
\mathrm{CD}_{3} \mathrm{CN}: \\
\mathrm{DMSO} \mathrm{d}_{6} \\
-30^{\circ} \mathrm{C}\end{array}$ & $\begin{array}{c}\mathbf{R F X}(\mathbf{B}) \\
\mathrm{CD}_{3} \mathrm{CN}: \\
\mathrm{DMSO}-\mathrm{d}_{6} \\
-30^{\circ} \mathrm{C}\end{array}$ & $\begin{array}{c}\text { RFX } \\
\mathrm{CD}_{3} \mathrm{CN} \\
25^{\circ} \mathrm{C}\end{array}$ \\
\hline 33 & $\begin{array}{c}3 \mathrm{H} \\
0.59(\mathrm{~d}) \\
{ }^{3} J_{\mathrm{H} 24 \mathrm{H} 33}=6.9\end{array}$ & $\begin{array}{c}3 \mathrm{H} \\
0.44(\mathrm{~d}) \\
{ }^{3} \mathrm{H}_{\mathrm{H} 24-\mathrm{H} 33}=6.9\end{array}$ & $\begin{array}{c}3 \mathrm{H} \\
0.60(\mathrm{~d}) \\
{ }^{3} \mathrm{H}_{\mathrm{H} 24-\mathrm{H} 33}=6.9\end{array}$ & $\begin{array}{c}3 \mathrm{H} \\
0.44(\mathrm{~d}) \\
{ }^{3} J_{\mathrm{H} 24-\mathrm{H} 33}=6.7\end{array}$ & $\begin{array}{c}3 \mathrm{H} \\
0.08(\mathrm{bs})\end{array}$ & $\begin{array}{c}3 \mathrm{H} \\
-0.05(\mathrm{~d}) \\
{ }^{3} \mathrm{~J}_{\mathrm{H} 24-\mathrm{H} 33}=6.1\end{array}$ & $\begin{array}{c}3 \mathrm{H} \\
-0.72(\mathrm{bs})\end{array}$ & $\begin{array}{c}3 \mathrm{H} \\
-0.08(\mathrm{~d}) \\
{ }^{3} J_{\mathrm{H} 24-\mathrm{H} 33}=7.2\end{array}$ \\
\hline 34 & $\begin{array}{c}3 \mathrm{H} \\
-0.31(\mathrm{~d}) \\
3 J_{\mathrm{H} 26-\mathrm{H} 36}=6.9 \\
\end{array}$ & $\begin{array}{c}3 \mathrm{H} \\
-0.25(\mathrm{~d}) \\
3 J_{\mathrm{H} 34-\mathrm{H} 26}=6.9 \\
\end{array}$ & $\begin{array}{c}3 \mathrm{H} \\
-0.30(\mathrm{~d}) \\
3 J_{\mathrm{H} 26-\mathrm{H} 36}=6.9 \\
\end{array}$ & $\begin{array}{c}3 \mathrm{H} \\
-0.27(\mathrm{~d}) \\
3 J_{\mathrm{H} 34-\mathrm{H} 26}=6.7 \\
\end{array}$ & $\begin{array}{c}3 \mathrm{H} \\
-0.63(\mathrm{~d}) \\
3 J_{\mathrm{H} 34-\mathrm{H} 26}=6.1 \\
\end{array}$ & $\begin{array}{c}3 \mathrm{H} \\
-0.68(\mathrm{~d}) \\
3 J_{\mathrm{H} 34-\mathrm{H} 26}=7.3 \\
\end{array}$ & $\begin{array}{c}3 \mathrm{H} \\
-0.88(\mathrm{bs})\end{array}$ & $\begin{array}{c}3 \mathrm{H} \\
-0.67(\mathrm{~d}) \\
{ }^{3} J_{\mathrm{H} 34-\mathrm{H} 26}=7 . \mathrm{C}\end{array}$ \\
\hline 35 & --- & -- & -- & -- & --- & -- & --- & --- \\
\hline 36 & $\begin{array}{c}3 \mathrm{H} \\
2.06(\mathrm{~s})\end{array}$ & $\begin{array}{c}3 \mathrm{H} \\
1.97(\mathrm{~s})\end{array}$ & $\begin{array}{c}3 \mathrm{H} \\
2.06(\mathrm{~s})\end{array}$ & $\begin{array}{c}3 \mathrm{H} \\
1.97(\mathrm{~s})\end{array}$ & $\begin{array}{c}3 \mathrm{H} \\
1.95(\mathrm{~s})\end{array}$ & $\begin{array}{c}3 \mathrm{H} \\
1.87(\mathrm{~s})\end{array}$ & $\begin{array}{c}3 \mathrm{H} \\
1.86(\mathrm{~s})\end{array}$ & $\begin{array}{c}3 \mathrm{H} \\
1.88(\mathrm{~s})\end{array}$ \\
\hline 37 & $\begin{array}{c}3 \mathrm{H} \\
3.04(\mathrm{~s})^{*}\end{array}$ & $\begin{array}{c}3 \mathrm{H} \\
2.90(\mathrm{~s})\end{array}$ & $\begin{array}{c}3 \mathrm{H} \\
3.04(\mathrm{~s})^{*}\end{array}$ & $\begin{array}{c}3 \mathrm{H} \\
2.89(\mathrm{~s})\end{array}$ & $\begin{array}{c}3 \mathrm{H} \\
2.97(\mathrm{~s})\end{array}$ & $\begin{array}{c}3 \mathrm{H} \\
2.90(\mathrm{~s})\end{array}$ & $\begin{array}{c}3 \mathrm{H} \\
2.80(\mathrm{~s})\end{array}$ & $\begin{array}{c}3 \mathrm{H} \\
2.92(\mathrm{~s})\end{array}$ \\
\hline 38 & $\begin{array}{c}1 \mathrm{H} \\
8.25(\mathrm{~s})\end{array}$ & $\begin{array}{c}1 \mathrm{H} \\
8.09(\mathrm{~s})\end{array}$ & $\begin{array}{c}1 \mathrm{H} \\
8.26(\mathrm{~s})\end{array}$ & $\begin{array}{c}1 \mathrm{H} \\
8.09(\mathrm{~s})\end{array}$ & $\begin{array}{c}1 \mathrm{H} \\
8.39(\mathrm{~d}) \\
{ }^{3} J_{\mathrm{H} 38-\mathrm{H} 39}=7.2\end{array}$ & $\begin{array}{c}1 \mathrm{H} \\
8.28(\mathrm{~d}) \\
{ }^{3} \mathrm{~J}_{\mathrm{H} 38-\mathrm{H} 39}=7.3\end{array}$ & $\begin{array}{c}1 \mathrm{H} \\
8.42(\mathrm{~d}) \\
{ }^{3} \mathrm{~J}_{\mathrm{H} 38-\mathrm{H} 39}=7.3\end{array}$ & $\begin{array}{c}1 \mathrm{H} \\
8.45(\mathrm{~d}) \\
{ }^{3} J_{\mathrm{H} 38-\mathrm{H} 39}=7.1\end{array}$ \\
\hline 39 & $\begin{array}{c}1 \mathrm{H} \\
3.09(\mathrm{~m}) \\
1 \mathrm{H} \\
3.17(\mathrm{~m})\end{array}$ & $\begin{array}{c}2 \mathrm{H} \\
3.30(\mathrm{~m})\end{array}$ & $\begin{array}{c}1 \mathrm{H} \\
3.08(\mathrm{~m}) \\
1 \mathrm{H} \\
3.18(\mathrm{~m})\end{array}$ & $\begin{array}{c}2 \mathrm{H} \\
3.51(\mathrm{~m})\end{array}$ & $\begin{array}{c}1 \mathrm{H} \\
7.05(\mathrm{~d})\end{array}$ & $\begin{array}{c}1 \mathrm{H} \\
7.15(\mathrm{~d})\end{array}$ & $\begin{array}{c}1 \mathrm{H} \\
7.32(\mathrm{~d})\end{array}$ & $\begin{array}{c}1 \mathrm{H} \\
7.16(\mathrm{~d})\end{array}$ \\
\hline 40 & $\begin{array}{c}1 \mathrm{H} \\
2.53(\mathrm{~m}) \\
1 \mathrm{H} \\
2.58(\mathrm{~m})\end{array}$ & $\begin{array}{c}2 \mathrm{H} \\
3.30(\mathrm{~m})\end{array}$ & $\begin{array}{c}1 \mathrm{H} \\
2.63(\mathrm{~m}) \\
1 \mathrm{H} \\
2.66(\mathrm{~m})\end{array}$ & $\begin{array}{c}2 \mathrm{H} \\
3.60(\mathrm{~m})\end{array}$ & --- & --- & --- & --- \\
\hline
\end{tabular}




\begin{tabular}{|c|c|c|c|c|c|c|c|c|}
\hline $\begin{array}{l}\text { Proton } \\
\text { number }\end{array}$ & $\begin{array}{c}\mathbf{R M P} \\
\mathrm{CDCl}_{3}\end{array}$ & $\begin{array}{c}\text { RMP } \\
\text { DMSO-d }_{6}\end{array}$ & $\begin{array}{c}\mathbf{R F P} \\
\mathrm{CDCl}_{3}\end{array}$ & $\begin{array}{c}\text { RFP } \\
\text { DMSO-d }_{6}\end{array}$ & $\begin{array}{c}\mathbf{R F X}(\mathbf{A}) \\
\mathrm{CDCl}_{3}\end{array}$ & $\begin{array}{c}\text { RFX(A) } \\
\mathrm{CD}_{3} \mathrm{CN}: \\
\mathrm{DMSO} \mathrm{d}_{6} \\
-30^{\circ} \mathrm{C}\end{array}$ & $\begin{array}{c}\mathbf{R F X}(\mathbf{B}) \\
\mathrm{CD}_{3} \mathrm{CN}: \\
\mathrm{DMSO} \mathrm{d}_{6} \\
-30^{\circ} \mathrm{C}\end{array}$ & $\begin{array}{c}\text { RFX } \\
\mathrm{CD}_{3} \mathrm{CN} \\
25^{\circ} \mathrm{C}\end{array}$ \\
\hline 41 & $\begin{array}{c}1 \mathrm{H} \\
2.53(\mathrm{~m}) \\
1 \mathrm{H} \\
2.58(\mathrm{~m})\end{array}$ & $\begin{array}{c}2 \mathrm{H} \\
3.30(\mathrm{~m})\end{array}$ & $\begin{array}{c}1 \mathrm{H} \\
2.63(\mathrm{~m}) \\
1 \mathrm{H} \\
2.66(\mathrm{~m})\end{array}$ & $\begin{array}{c}2 \mathrm{H} \\
3.60(\mathrm{~m})\end{array}$ & $\begin{array}{c}1 \mathrm{H} \\
7.32(\mathrm{~s})\end{array}$ & $\begin{array}{c}1 \mathrm{H} \\
7.90(\mathrm{~s})\end{array}$ & $\begin{array}{c}1 \mathrm{H} \\
7.94(\mathrm{~s})\end{array}$ & $\begin{array}{c}1 \mathrm{H} \\
7.64(\mathrm{~s})\end{array}$ \\
\hline 42 & $\begin{array}{c}1 \mathrm{H} \\
3.09(\mathrm{~m}) \\
1 \mathrm{H} \\
3.17(\mathrm{~m})\end{array}$ & $\begin{array}{c}2 \mathrm{H} \\
3.30(\mathrm{~m})\end{array}$ & $\begin{array}{c}1 \mathrm{H} \\
3.08(\mathrm{~m}) \\
1 \mathrm{H} \\
3.18(\mathrm{~m})\end{array}$ & $\begin{array}{c}2 \mathrm{H} \\
3.51(\mathrm{~m})\end{array}$ & --- & --- & --- & --- \\
\hline 43 & $\begin{array}{c}3 \mathrm{H} \\
2.34(\mathrm{~s})\end{array}$ & $\begin{array}{c}3 \mathrm{H} \\
2.79(\mathrm{~s})\end{array}$ & $\begin{array}{c}3 \mathrm{H} \\
2.54(\mathrm{~s})\end{array}$ & $\begin{array}{c}3 \mathrm{H} \\
3.47(\mathrm{~m})\end{array}$ & $\begin{array}{c}3 \mathrm{H} \\
2.62(\mathrm{~s})\end{array}$ & $\begin{array}{c}3 \mathrm{H} \\
2.51(\mathrm{~s})\end{array}$ & $\begin{array}{c}3 \mathrm{H} \\
2.51(\mathrm{~s})\end{array}$ & $\begin{array}{c}3 \mathrm{H} \\
2.55(\mathrm{~s})\end{array}$ \\
\hline 44 & --- & --- & $\begin{array}{c}1 \mathrm{H} \\
1.41(\mathrm{~m}) \\
1 \mathrm{H} \\
1.86(\mathrm{~m})\end{array}$ & $\begin{array}{c}1 \mathrm{H} \\
1.62(\mathrm{~m}) \\
1 \mathrm{H} \\
1.99(\mathrm{~m})\end{array}$ & --- & --- & --- & --- \\
\hline 45 & --- & --- & $\begin{array}{c}1 \mathrm{H} \\
1.56(\mathrm{~m}) \\
1 \mathrm{H} \\
1.71(\mathrm{~m})\end{array}$ & $\begin{array}{c}1 \mathrm{H} \\
1.55(\mathrm{~m}) \\
1 \mathrm{H} \\
1.69(\mathrm{~m})\end{array}$ & --- & --- & --- & --- \\
\hline
\end{tabular}




\begin{tabular}{|c|c|c|c|c|c|c|c|c|}
\hline $\begin{array}{l}\text { Proton } \\
\text { number }\end{array}$ & $\begin{array}{c}\mathbf{R M P} \\
\mathrm{CDCl}_{3}\end{array}$ & $\begin{array}{c}\text { RMP } \\
\text { DMSO-d }_{6}\end{array}$ & $\begin{array}{c}\mathbf{R F P} \\
\mathrm{CDCl}_{3}\end{array}$ & $\begin{array}{c}\text { RFP } \\
\text { DMSO-d }_{6}\end{array}$ & $\begin{array}{c}\mathbf{R F X}(\mathbf{A}) \\
\mathrm{CDCl}_{3}\end{array}$ & $\begin{array}{c}\mathbf{R F X}(\mathbf{A}) \\
\mathrm{CD}_{3} \mathrm{CN}: \\
\mathrm{DMSO} \mathrm{d}_{6} \\
-30^{\circ} \mathrm{C}\end{array}$ & $\begin{array}{c}\mathbf{R F X}(\mathbf{B}) \\
\mathrm{CD}_{3} \mathrm{CN}: \\
\mathrm{DMSO} \mathrm{d}_{6} \\
-30^{\circ} \mathrm{C}\end{array}$ & $\begin{array}{c}\text { RFX } \\
\mathrm{CD}_{3} \mathrm{CN} \\
25^{\circ} \mathrm{C}\end{array}$ \\
\hline 46 & --- & --- & $\begin{array}{c}1 \mathrm{H} \\
1.56(\mathrm{~m}) \\
1 \mathrm{H} \\
1.71(\mathrm{~m})\end{array}$ & $\begin{array}{c}1 \mathrm{H} \\
1.55(\mathrm{~m}) \\
1 \mathrm{H} \\
1.69(\mathrm{~m})\end{array}$ & --- & --- & --- & --- \\
\hline 47 & --- & --- & $\begin{array}{c}1 \mathrm{H} \\
1.41(\mathrm{~m}) \\
1 \mathrm{H} \\
1.86(\mathrm{~m})\end{array}$ & $\begin{array}{c}1 \mathrm{H} \\
1.62(\mathrm{~m}) \\
1 \mathrm{H} \\
1.99(\mathrm{~m})\end{array}$ & --- & --- & --- & --- \\
\hline $\mathrm{OH}-1$ & $\begin{array}{c}1 \mathrm{H} \\
13.19(\mathrm{~s})\end{array}$ & $\begin{array}{c}1 \mathrm{H} \\
15.61(\mathrm{bs})\end{array}$ & $\begin{array}{c}1 \mathrm{H} \\
13.24(\mathrm{~s})\end{array}$ & $\begin{array}{c}1 \mathrm{H} \\
15.63(\mathrm{bs})\end{array}$ & $\begin{array}{c}1 \mathrm{H} \\
16.83(\mathrm{bs})\end{array}$ & $\begin{array}{c}1 \mathrm{H} \\
16.87(\mathrm{~s})\end{array}$ & $\begin{array}{c}1 \mathrm{H} \\
17.81(\mathrm{~s})\end{array}$ & $\begin{array}{c}1 \mathrm{H} \\
17.22(\mathrm{~s})\end{array}$ \\
\hline $\mathrm{OH}-4$ & $\begin{array}{c}1 \mathrm{H} \\
11.98(\mathrm{~s})\end{array}$ & $\begin{array}{c}1 \mathrm{H} \\
12.52(\mathrm{~s})\end{array}$ & $\begin{array}{c}1 \mathrm{H} \\
12.00(\mathrm{~s})\end{array}$ & $\begin{array}{c}1 \mathrm{H} \\
12.52(\mathrm{~s})\end{array}$ & --- & --- & --- & --- \\
\hline $\mathrm{OH}-8$ & $\begin{array}{c}1 \mathrm{H} \\
13.30(\mathrm{vbs})\end{array}$ & --- & $\begin{array}{c}1 \mathrm{H} \\
13.24(\mathrm{vbs})\end{array}$ & --- & --- & --- & --- & --- \\
\hline $\mathrm{OH}-21$ & $\begin{array}{c}1 \mathrm{H} \\
3.48(\mathrm{bs})\end{array}$ & $\begin{array}{c}1 \mathrm{H} \\
5.02(\mathrm{~s})\end{array}$ & $\begin{array}{c}1 \mathrm{H} \\
3.47(\mathrm{bs})\end{array}$ & $\begin{array}{c}1 \mathrm{H} \\
5.08(\mathrm{~s})\end{array}$ & $\begin{array}{c}1 \mathrm{H} \\
3.72(\mathrm{bs})\end{array}$ & $\begin{array}{c}1 \mathrm{H} \\
4.66(\mathrm{~s})\end{array}$ & $\begin{array}{c}1 \mathrm{H} \\
5.28(\mathrm{bs})\end{array}$ & $\begin{array}{c}1 \mathrm{H} \\
3.38(\mathrm{bs})\end{array}$ \\
\hline $\mathrm{OH}-23$ & $\begin{array}{c}1 \mathrm{H} \\
3.69(\mathrm{bs})\end{array}$ & $\begin{array}{c}1 \mathrm{H} \\
4.20(\mathrm{~d}) \\
J_{\mathrm{HO}-\mathrm{H} 23}=8.6\end{array}$ & $\begin{array}{c}1 \mathrm{H} \\
3.64(\mathrm{bs})\end{array}$ & $\begin{array}{c}1 \mathrm{H} \\
4.14(\mathrm{~d}) \\
{ }^{3} J_{\mathrm{HO}-\mathrm{H} 23}=8.5\end{array}$ & $\begin{array}{c}1 \mathrm{H} \\
3.86(\mathrm{~d}) \\
{ }^{3} \mathrm{~J}_{\mathrm{HO}-\mathrm{H} 23}=5.9\end{array}$ & $\begin{array}{c}1 \mathrm{H} \\
4.60(\mathrm{~s})\end{array}$ & $\begin{array}{c}1 \mathrm{H} \\
3.88(\mathrm{bs})\end{array}$ & $\begin{array}{c}1 \mathrm{H} \\
3.60(\mathrm{~d}) \\
J_{\mathrm{H}} \mathrm{HO}_{\mathrm{H} 22}=7.1\end{array}$ \\
\hline NH (amid) & $\begin{array}{c}1 \mathrm{H} \\
13.19(\mathrm{~s})\end{array}$ & $\begin{array}{c}1 \mathrm{H} \\
8.84(\mathrm{~s})\end{array}$ & $\begin{array}{c}1 \mathrm{H} \\
13.24(\mathrm{~s})\end{array}$ & $\begin{array}{c}1 \mathrm{H} \\
8.78(\mathrm{~s})\end{array}$ & $\begin{array}{c}1 \mathrm{H} \\
8.65(\mathrm{~s})\end{array}$ & $\begin{array}{c}1 \mathrm{H} \\
9.71(\mathrm{~s})\end{array}$ & $\begin{array}{c}1 \mathrm{H} \\
9.67(\mathrm{~s})\end{array}$ & $\begin{array}{c}1 \mathrm{H} \\
8.42(\mathrm{~s})\end{array}$ \\
\hline $\mathrm{N}^{+} \mathrm{H}$ & --- & $\begin{array}{c}1 \mathrm{H} \\
9.54(\mathrm{vbs})\end{array}$ & --- & $\begin{array}{c}1 \mathrm{H} \\
9.34(\mathrm{bs}) \\
\end{array}$ & $\begin{array}{c}1 \mathrm{H} \\
14.71(\mathrm{bs})\end{array}$ & $\begin{array}{c}1 \mathrm{H} \\
14.14(\mathrm{~s}) \\
\end{array}$ & $\begin{array}{c}1 \mathrm{H} \\
14.26(\mathrm{~s}) \\
\end{array}$ & $\begin{array}{c}1 \mathrm{H} \\
14.40(\mathrm{~s}) \\
\end{array}$ \\
\hline
\end{tabular}




\section{Table S2}

${ }^{13} \mathrm{C}$ NMR data of Rifampicin (RMP), Rifapentin (RFP) and Rifaximin (RFX) assigned on the basis of ${ }^{1} \mathrm{H}_{-}{ }^{13} \mathrm{C} \mathrm{HSQC}^{1} \mathrm{H}_{-}{ }^{13} \mathrm{C} \mathrm{HMBC}$, ${ }^{1} \mathrm{H}-{ }^{1} \mathrm{H}$ COSY and ${ }^{1} \mathrm{H}-{ }^{1} \mathrm{H}$ NOESY correlation spectra.

\begin{tabular}{|c|c|c|c|c|c|c|c|c|}
\hline $\begin{array}{l}\text { Carbon } \\
\text { atom } \\
\text { number }\end{array}$ & $\begin{array}{c}\mathbf{R M P} \\
\mathrm{CDCl}_{3}\end{array}$ & $\begin{array}{c}\text { RMP } \\
\text { DMSO-d }_{6}\end{array}$ & $\begin{array}{c}\mathbf{R F P} \\
\mathrm{CDCl}_{3}\end{array}$ & $\begin{array}{c}\text { RFP } \\
\text { DMSO-d }_{6}\end{array}$ & $\begin{array}{c}\mathbf{R F X}(\mathbf{A}) \\
\mathrm{CDCl}_{3}\end{array}$ & $\begin{array}{c}\mathbf{R F X}(\mathbf{A}) \\
\mathrm{CD}_{3} \mathrm{CN}: \\
\mathrm{DMSO}^{-} \mathrm{d}_{6} \\
-30^{\circ} \mathrm{C} \\
\end{array}$ & $\begin{array}{c}\mathbf{R F X ( B )} \\
\mathrm{CD}_{3} \mathrm{CN}: \\
\mathrm{DMSO} \mathrm{d}_{6} \\
-30^{\circ} \mathrm{C} \\
\end{array}$ & $\begin{array}{c}\text { RFX } \\
\mathrm{CD}_{3} \mathrm{CN} \\
25^{\circ} \mathrm{C}\end{array}$ \\
\hline 1 & 138.5 & 148.6 & 138.6 & 148.6 & 155.1 & 155.6 & 157.1 & 157.2 \\
\hline 2 & 120.4 & 117.2 & 120.5 & 117.2 & 112.0 & 111.6 & 112.2 & 112.8 \\
\hline 3 & 110.8 & 114.2 & 111.0 & 114.2 & 123.0 & 123.1 & 123.3 & 124.2 \\
\hline 4 & 147.9 & 145.4 & 147.9 & 145.4 & 119.2 & 118.4 & 119.0 & 120.1 \\
\hline 5 & 104.5 & 98.5 & 104.5 & 98.5 & 97.8 & 97.7 & 99.2 & 98.8 \\
\hline 6 & 174.4 & 171.7 & 174.5 & 171.7 & 171.9 & 171.4 & 171.5 & 172.6 \\
\hline 7 & 106.0 & 101.1 & 106.1 & 101.0 & 104.1 & 103.1 & 103.8 & 104.3 \\
\hline 8 & 169.2 & 184.2 & 169.2 & 184.2 & 182.2 & 182.2 & 181.8 & 183.0 \\
\hline 9 & 112.8 & 115.5 & 112.8 & 115.3 & 103.8 & 103.2 & 102.3 & 103.9 \\
\hline 10 & 117.9 & 116.9 & 117.9 & 117.1 & 115.0 & 115.4 & 115.8 & 116.5 \\
\hline 11 & 195.4 & 184.5 & 195.4 & 184.4 & 188.8 & 188.7 & 188.4 & 189.7 \\
\hline 12 & 108.7 & 108.5 & 108.8 & 108.6 & 109.0 & 108.7 & 109.7 & 109.8 \\
\hline 13 & 21.4 & 22.0 & 21.5 & 22.0 & 21.4 & 21.4 & 22.2 & 21.2 \\
\hline 14 & 7.5 & 7.4 & 7.5 & 7.4 & 7.0 & 7.4 & 7.4 & 7.4 \\
\hline 15 & 169.6 & 166.2 & 169.7 & 166.3 & 170.6 & 169.2 & 170.0 & 171.2 \\
\hline 16 & 129.3 & 132.1 & 129.3 & 132.1 & 129.1 & 129.8 & 131.5 & 130.9 \\
\hline 17 & 135.1 & 132.0 & 135.2 & 132.0 & 136.0 & 135.6 & 132.5 & 136.0 \\
\hline 18 & 123.2 & 127.5 & 123.2 & 127.9 & 125.3 & 126.4 & 126.6 & 127.0 \\
\hline 19 & 142.7 & 138.2 & 142.7 & 138.0 & 141.6 & 139.8 & 140.3 & 141.7 \\
\hline 20 & 38.5 & 38.4 & 38.6 & 38.3 & 37.7 & 38.2 & 37.8 & 39.4 \\
\hline 21 & 70.6 & 72.1 & 70.6 & 72.2 & 72.8 & 71.4 & 73.2 & 73.5 \\
\hline 22 & 33.3 & 32.9 & 33.4 & 32.8 & 32.9 & 37.8 & 32.5 & 33.7 \\
\hline
\end{tabular}




\begin{tabular}{|c|c|c|c|c|c|c|c|c|}
\hline $\begin{array}{l}\text { Carbon } \\
\text { atom } \\
\text { number }\end{array}$ & $\begin{array}{r}\mathbf{R M P} \\
\mathrm{CDCl}_{3}\end{array}$ & $\begin{array}{c}\text { RMP } \\
\text { DMSO-d }_{6}\end{array}$ & $\begin{array}{c}\mathbf{R F P} \\
\mathrm{CDCl}_{3}\end{array}$ & $\begin{array}{c}\text { RFP } \\
\text { DMSO-d }_{6}\end{array}$ & $\begin{array}{c}\mathbf{R F X ( A )} \\
\mathrm{CDCl}_{3}\end{array}$ & $\begin{array}{c}\text { RFX(A) } \\
\mathrm{CD}_{3} \mathrm{CN}: \\
\mathrm{DMSO}-\mathrm{d}_{6} \\
-30{ }^{\circ} \mathrm{C}\end{array}$ & $\begin{array}{c}\text { RFX(B) } \\
\mathrm{CD}_{3} \mathrm{CN}: \\
\mathrm{DMSO} \mathrm{d}_{6} \\
-30{ }^{\circ} \mathrm{C}\end{array}$ & $\begin{array}{r}\text { RFX } \\
\mathrm{CD}_{3} \mathrm{CN} \\
25^{\circ} \mathrm{C}\end{array}$ \\
\hline 23 & 76.8 & 76.2 & 76.9 & 76.1 & 76.7 & 75.9 & 75.4 & 77.1 \\
\hline 24 & 37.5 & 38.0 & 37.5 & 38.1 & 36.9 & 37.1 & 37.4 & 38.2 \\
\hline 25 & 74.4 & 73.6 & 74.4 & 73.6 & 73.9 & 72.9 & 72.6 & 74.2 \\
\hline 26 & 39.5 & 40.1 & 39.5 & 40.1 & 38.6 & 39.6 & 40.0 & 39.9 \\
\hline 27 & 76.7 & 76.5 & 76.7 & 76.4 & 77.8 & 76.5 & 76.1 & 78.1 \\
\hline 28 & 118.6 & 117.6 & 118.6 & 117.6 & 115.6 & 115.0 & 117.4 & 117.6 \\
\hline 29 & 142.6 & 142.7 & 142.7 & 142.8 & 142.1 & 141.9 & 143.7 & 143.0 \\
\hline 30 & 20.5 & 20.5 & 20.7 & 20.5 & 20.4 & 20.1 & 20.3 & 20.6 \\
\hline 31 & 17.8 & 17.7 & 17.8 & 17.8 & 17.6 & 17.3 & 18.1 & 17.8 \\
\hline 32 & 10.8 & 11.3 & 10.9 & 11.3 & 10.7 & 10.5 & 10.7 & 11.1 \\
\hline 33 & 8.4 & 8.6 & 8.4 & 8.7 & 8.2 & 8.1 & 6.2 & 8.2 \\
\hline 34 & 8.9 & 8.9 & 9.0 & 9.0 & 8.0 & 8.4 & 8.7 & 8.6 \\
\hline 35 & 172.0 & 169.5 & 172.1 & 169.4 & 171.9 & 170.5 & 171.2 & 172.1 \\
\hline 36 & 20.5 & 20.6 & 20.7 & 20.7 & 20.8 & 20.7 & 20.5 & 21.0 \\
\hline 37 & 57.0 & 55.6 & 57.1 & 55.6 & 57.0 & 53.6 & 55.6 & 57.0 \\
\hline 38 & 134.4 & 137.6 & 134.3 & 137.6 & 128.3 & 127.1 & 126.8 & 128.6 \\
\hline 39 & 50.2 & 47.6 & 50.4 & 47.2 & 117.6 & 118.4 & 118.7 & 119.1 \\
\hline 40 & 53.9 & 51.2 & 51.0 & 48.8 & 147.8 & 147.9 & 148.2 & 149.3 \\
\hline 41 & 53.9 & 51.2 & 51.0 & 48.8 & 110.0 & 111.3 & 112.7 & 111.8 \\
\hline 42 & 50.2 & 47.6 & 50.4 & 47.2 & 138.9 & 139.3 & 139.5 & 140.3 \\
\hline 43 & 45.9 & 42.2 & 67.1 & 66.3 & 22.4 & 21.8 & 21.6 & 22.2 \\
\hline 44 & -- & -- & 30.5 & 27.6 & -- & -- & -- & -- \\
\hline 45 & -- & -- & 24.2 & 23.5 & -- & -- & -- & -- \\
\hline 46 & -- & -- & 24.2 & 23.5 & -- & -- & -- & -- \\
\hline 47 & -- & --- & 30.6 & 27.7 & -- & -- & -- & --- \\
\hline
\end{tabular}




\section{Table S3}

${ }^{1} \mathrm{H}$ NMR data of Rifabutin (RFB) assigned on the basis of ${ }^{1} \mathrm{H}-{ }^{13} \mathrm{C}$ HSQC, ${ }^{1} \mathrm{H}-{ }^{13} \mathrm{C}$ HMBC, ${ }^{1} \mathrm{H}-{ }^{1} \mathrm{H}$ COSY and ${ }^{1} \mathrm{H}-{ }^{1} \mathrm{H}$ NOESY correlation spectra.

\begin{tabular}{|c|c|c|c|c|c|c|c|c|c|}
\hline $\begin{array}{l}\text { Proton } \\
\text { number }\end{array}$ & $\begin{array}{c}\mathbf{R F B}(\mathbf{A}) \\
\mathrm{CDCl}_{3}\end{array}$ & $\begin{array}{c}\mathbf{R F B}(\mathbf{A}) \\
\mathrm{DMSO}^{-\mathrm{d}_{6}}\end{array}$ & $\begin{array}{c}\mathbf{R F B}(\mathbf{C}) \\
\mathrm{DMSO}^{-d_{6}}\end{array}$ & $\begin{array}{c}\mathbf{R F B}(\mathbf{A}) \\
\mathrm{CD}_{3} \mathrm{CN}\end{array}$ & $\begin{array}{c}\mathbf{R F B}(\mathbf{C}) \\
\mathrm{CD}_{3} \mathrm{CN}\end{array}$ & $\begin{array}{c}\mathbf{R F B}(\mathbf{A}) \\
\mathrm{CD}_{3} \mathrm{CN} \\
-20{ }^{\circ} \mathrm{C} \\
\end{array}$ & $\begin{array}{c}\mathbf{R F B ( C )} \\
\mathrm{CD}_{3} \mathrm{CN} \\
-20{ }^{\circ} \mathrm{C} \\
\end{array}$ & $\begin{array}{c}\mathbf{R F B}(\mathbf{A}) \\
\mathrm{CD}_{3} \mathrm{CN}: \\
\mathrm{DMSO}^{-\mathrm{d}_{6}}\end{array}$ & $\begin{array}{c}\mathbf{R F B}(\mathbf{C}) \\
\mathrm{CD}_{3} \mathrm{CN}: \\
\mathrm{DMSO}^{-d_{6}}\end{array}$ \\
\hline 1 & -- & -- & --- & -- & --- & -- & -- & -- & -- \\
\hline 2 & -- & -- & -- & -- & -- & -- & -- & -- & -- \\
\hline 3 & -- & -- & -- & -- & -- & -- & -- & -- & -- \\
\hline 4 & -- & -- & -- & -- & -- & -- & -- & -- & -- \\
\hline 5 & -- & -- & -- & -- & -- & -- & -- & -- & -- \\
\hline 6 & -- & -- & -- & -- & -- & -- & -- & -- & -- \\
\hline 7 & -- & -- & -- & -- & -- & -- & -- & -- & -- \\
\hline 8 & -- & -- & -- & -- & -- & -- & -- & -- & -- \\
\hline 9 & -- & -- & -- & -- & -- & -- & -- & -- & -- \\
\hline 10 & -- & -- & -- & -- & -- & -- & -- & -- & -- \\
\hline 11 & -- & -- & -- & -- & -- & -- & -- & -- & -- \\
\hline 12 & -- & -- & -- & --- & -- & -- & -- & -- & -- \\
\hline 14 & $\begin{array}{c}3 \mathrm{H} \\
2.33(\mathrm{~s})\end{array}$ & $\begin{array}{c}3 \mathrm{H} \\
2.20(\mathrm{~s})\end{array}$ & $\begin{array}{c}3 \mathrm{H} \\
2.10(\mathrm{~s})\end{array}$ & $\begin{array}{c}3 \mathrm{H} \\
2.21(\mathrm{~s})\end{array}$ & $\begin{array}{c}3 \mathrm{H} \\
2.11(\mathrm{~s})\end{array}$ & $\begin{array}{c}3 \mathrm{H} \\
2.00(\mathrm{~s})\end{array}$ & $\begin{array}{c}3 \mathrm{H} \\
1.99(\mathrm{~s})\end{array}$ & $\begin{array}{c}3 \mathrm{H} \\
2.22(\mathrm{~s})\end{array}$ & $\begin{array}{c}3 \mathrm{H} \\
2.13(\mathrm{~s})\end{array}$ \\
\hline 15 & -- & -- & -- & -- & -- & -- & -- & -- & -- \\
\hline 16 & -- & -- & -- & -- & -- & -- & -- & -- & -- \\
\hline 17 & $\begin{array}{c}1 \mathrm{H} \\
6.25(\mathrm{~d}) \\
{ }^{3} J_{\mathrm{H} 17-\mathrm{H} 18}=10.4\end{array}$ & $\begin{array}{c}1 \mathrm{H} \\
6.28(\mathrm{~d}) \\
{ }^{3} J_{\mathrm{H} 17-\mathrm{H} 18} \\
=10.7\end{array}$ & $\begin{array}{c}1 \mathrm{H} \\
5.76(\mathrm{~d}) \\
{ }^{3} J_{\mathrm{H} 17-\mathrm{H} 18} \\
=11.5\end{array}$ & $\begin{array}{c}1 \mathrm{H} \\
6.24(\mathrm{~d}) \\
{ }^{3} J_{\mathrm{H} 17-\mathrm{H} 18} \\
=10.6\end{array}$ & $\begin{array}{c}1 \mathrm{H} \\
5.85(\mathrm{~d}) \\
{ }^{3} J_{\mathrm{H} 17-\mathrm{H} 18} \\
=11.5\end{array}$ & $\begin{array}{c}1 \mathrm{H} \\
6.19(\mathrm{~d}) \\
{ }^{3} J_{\mathrm{H} 17-\mathrm{H} 18} \\
=10.8\end{array}$ & $\begin{array}{c}1 \mathrm{H} \\
5.82(\mathrm{~m})\end{array}$ & $\begin{array}{c}1 \mathrm{H} \\
6.27(\mathrm{~d}) \\
{ }^{3} J_{\mathrm{H} 17-\mathrm{H} 18} \\
=11.0\end{array}$ & $\begin{array}{c}1 \mathrm{H} \\
5.78(\mathrm{~d}) \\
{ }^{3} J_{\mathrm{H} 17-\mathrm{H} 18} \\
=11.7\end{array}$ \\
\hline
\end{tabular}




\begin{tabular}{|c|c|c|c|c|c|c|c|c|c|}
\hline $\begin{array}{l}\text { Proton } \\
\text { number }\end{array}$ & $\begin{array}{c}\mathbf{R F B}(\mathbf{A}) \\
\mathrm{CDCl}_{3}\end{array}$ & $\begin{array}{c}\text { RFB(A) } \\
\text { DMSO-d }_{6}\end{array}$ & $\begin{array}{c}\text { RFB(C) } \\
\text { DMSO-d }_{6}\end{array}$ & $\begin{array}{l}\mathbf{R F B}(\mathbf{A}) \\
\mathrm{CD}_{3} \mathrm{CN}\end{array}$ & $\begin{array}{l}\mathbf{R F B}(\mathbf{C}) \\
\mathrm{CD}_{3} \mathrm{CN}\end{array}$ & $\begin{array}{c}\mathbf{R F B ( A )} \\
\mathrm{CD}_{3} \mathrm{CN} \\
-20{ }^{\circ} \mathrm{C} \\
\end{array}$ & $\begin{array}{c}\mathbf{R F B}(\mathbf{C}) \\
\mathrm{CD}_{3} \mathrm{CN} \\
-20^{\circ} \mathrm{C} \\
\end{array}$ & $\begin{array}{c}\text { RFB(A) } \\
\mathrm{CD}_{3} \mathrm{CN}: \\
\mathrm{DMSO} \mathrm{d}_{6}\end{array}$ & $\begin{array}{c}\mathbf{R F B}(\mathbf{C}) \\
\mathrm{CD}_{3} \mathrm{CN}: \\
\mathrm{DMSO}^{-\mathrm{d}_{6}}\end{array}$ \\
\hline 18 & $\begin{array}{c}1 \mathrm{H} \\
6.37(\mathrm{dd}) \\
{ }^{3} J_{\mathrm{H} 18-\mathrm{H} 19}=15.8\end{array}$ & $\begin{array}{c}1 \mathrm{H} \\
6.60(\mathrm{dd}) \\
{ }^{3} J_{\mathrm{H} 18-\mathrm{H} 19} \\
=15.9\end{array}$ & $\begin{array}{c}1 \mathrm{H} \\
5.82(\mathrm{~m})\end{array}$ & $\begin{array}{c}1 \mathrm{H} \\
6.42(\mathrm{dd}) \\
{ }^{3} J_{\mathrm{H} 18-\mathrm{H} 19} \\
=15.9\end{array}$ & $\begin{array}{c}1 \mathrm{H} \\
5.77(\mathrm{dd}) \\
{ }^{3} J_{\mathrm{H} 18-\mathrm{H} 19} \\
=14.4\end{array}$ & $\begin{array}{c}1 \mathrm{H} \\
6.48(\mathrm{~m})\end{array}$ & $\begin{array}{c}1 \mathrm{H} \\
5.70(\mathrm{~m})\end{array}$ & $\begin{array}{c}1 \mathrm{H} \\
6.59(\mathrm{dd}) \\
{ }^{3} J_{\mathrm{H} 18-\mathrm{H} 19} \\
=16.1\end{array}$ & $\begin{array}{c}1 \mathrm{H} \\
5.85(\mathrm{~m})\end{array}$ \\
\hline 19 & $\begin{array}{c}1 \mathrm{H} \\
6.03(\mathrm{dd}) \\
{ }^{3} J_{\mathrm{H} 19-\mathrm{H} 20}=6.7\end{array}$ & $\begin{array}{c}1 \mathrm{H} \\
6.00(\mathrm{dd}) \\
{ }^{3} J_{\mathrm{H} 19-\mathrm{H} 20}=7.5\end{array}$ & $\begin{array}{c}1 \mathrm{H} \\
5.14(\mathrm{~m})\end{array}$ & $\begin{array}{c}1 \mathrm{H} \\
6.00(\mathrm{dd}) \\
{ }^{3} J_{\mathrm{H} 19-\mathrm{H} 20}=7.0\end{array}$ & $\begin{array}{c}1 \mathrm{H} \\
5.10(\mathrm{dd}) \\
{ }^{3} J_{\mathrm{H} 19-\mathrm{H} 20}=10.0\end{array}$ & $\begin{array}{c}1 \mathrm{H} \\
5.99(\mathrm{dd}) \\
{ }^{3} J_{\mathrm{H} 18-\mathrm{H} 19} \\
=16.0 \\
{ }^{3} J_{\mathrm{H} 19-\mathrm{H} 20}=7.0\end{array}$ & $\begin{array}{c}1 \mathrm{H} \\
5.03(\mathrm{~m})\end{array}$ & $\begin{array}{c}1 \mathrm{H} \\
6.02(\mathrm{dd}) \\
{ }^{3} J_{\mathrm{H} 19-\mathrm{H} 20}=7.5\end{array}$ & $\begin{array}{c}1 \mathrm{H} \\
5.14(\mathrm{~m})\end{array}$ \\
\hline 20 & $\begin{array}{c}1 \mathrm{H} \\
2.38(\mathrm{~m})\end{array}$ & $\begin{array}{c}1 \mathrm{H} \\
2.25(\mathrm{~m})\end{array}$ & $\begin{array}{c}1 \mathrm{H} \\
1.84(\mathrm{~m})\end{array}$ & $\begin{array}{c}1 \mathrm{H} \\
2.25(\mathrm{~m})\end{array}$ & $\begin{array}{c}1 \mathrm{H} \\
1.83(\mathrm{~m})\end{array}$ & $\begin{array}{c}1 \mathrm{H} \\
2.30(\mathrm{~m})\end{array}$ & $\begin{array}{c}1 \mathrm{H} \\
1.71(\mathrm{~m})\end{array}$ & $\begin{array}{c}1 \mathrm{H} \\
2.31(\mathrm{~m})\end{array}$ & $\begin{array}{c}1 \mathrm{H} \\
1.84(\mathrm{~m})\end{array}$ \\
\hline 21 & $\begin{array}{c}1 \mathrm{H} \\
3.67(\mathrm{~d}) \\
{ }^{3} \mathrm{H}_{2} 2 \mathrm{-H} 2 \mathrm{2} 2=9.8\end{array}$ & $\begin{array}{c}1 \mathrm{H} \\
3.69(\mathrm{~d}) \\
{ }^{3} J_{\mathrm{H} 20-\mathrm{H} 22}=8.8\end{array}$ & $\begin{array}{c}1 \mathrm{H} \\
3.10(\mathrm{~m})\end{array}$ & $\begin{array}{c}1 \mathrm{H} \\
3.72(\mathrm{~d}) \\
3 J_{\mathrm{H} 20}-\mathrm{H} 21=9.7\end{array}$ & $\begin{array}{c}1 \mathrm{H} \\
3.20(\mathrm{~d}) \\
{ }^{3} J_{\mathrm{H} 20-\mathrm{H} 21}=9.9\end{array}$ & $\begin{array}{c}1 \mathrm{H} \\
3.79(\mathrm{~d}) \\
3_{\mathrm{H} 20-\mathrm{H} 21}=10.0\end{array}$ & $\begin{array}{c}1 \mathrm{H} \\
3.12(\mathrm{~m})\end{array}$ & $\begin{array}{c}1 \mathrm{H} \\
3.73(\mathrm{~m})\end{array}$ & \\
\hline 22 & $\begin{array}{c}1 \mathrm{H} \\
1.76 \\
(\mathrm{~m})^{\operatorname{cosy} . \mathrm{HSQ}} \\
\mathrm{C}\end{array}$ & $\begin{array}{c}1 \mathrm{H} \\
1.66(\mathrm{~m})\end{array}$ & $\begin{array}{c}1 \mathrm{H} \\
1.62(\mathrm{~m})\end{array}$ & $\begin{array}{c}1 \mathrm{H} \\
1.66(\mathrm{~m})\end{array}$ & $\begin{array}{c}1 \mathrm{H} \\
1.66(\mathrm{~m})\end{array}$ & $\begin{array}{c}1 \mathrm{H} \\
1.73(\mathrm{~m})\end{array}$ & $\begin{array}{c}1 \mathrm{H} \\
1.62(\mathrm{~m})\end{array}$ & $\begin{array}{c}1 \mathrm{H} \\
1.67(\mathrm{~m})\end{array}$ & $\begin{array}{c}1 \mathrm{H} \\
1.58(\mathrm{~m})\end{array}$ \\
\hline 23 & $\begin{array}{c}1 \mathrm{H} \\
2.99(\mathrm{~m})\end{array}$ & $\begin{array}{c}1 \mathrm{H} \\
2.85(\mathrm{~m})\end{array}$ & $\begin{array}{c}1 \mathrm{H} \\
2.80(\mathrm{~m})\end{array}$ & $\begin{array}{c}1 \mathrm{H} \\
2.87(\mathrm{~m})\end{array}$ & $\begin{array}{c}1 \mathrm{H} \\
2.79(\mathrm{~m})\end{array}$ & $\begin{array}{c}1 \mathrm{H} \\
2.96(\mathrm{~m})\end{array}$ & $\begin{array}{c}1 \mathrm{H} \\
2.79(\mathrm{~m})\end{array}$ & $\begin{array}{c}1 \mathrm{H} \\
2.82(\mathrm{~m})\end{array}$ & $\begin{array}{c}1 \mathrm{H} \\
2.79(\mathrm{~m})\end{array}$ \\
\hline 24 & $\begin{array}{c}1 \mathrm{H} \\
1.45(\mathrm{~m})\end{array}$ & $\begin{array}{c}1 \mathrm{H} \\
1.41(\mathrm{~m})\end{array}$ & $\begin{array}{c}1 \mathrm{H} \\
1.57(\mathrm{~m})\end{array}$ & $\begin{array}{c}1 \mathrm{H} \\
1.45(\mathrm{~m})\end{array}$ & $\begin{array}{c}1 \mathrm{H} \\
1.55(\mathrm{~m})\end{array}$ & $\begin{array}{c}1 \mathrm{H} \\
1.45(\mathrm{~m})\end{array}$ & $\begin{array}{c}1 \mathrm{H} \\
1.47(\mathrm{~m})\end{array}$ & $\begin{array}{c}1 \mathrm{H} \\
1.43(\mathrm{~m})\end{array}$ & $\begin{array}{c}1 \mathrm{H} \\
1.58(\mathrm{~m})\end{array}$ \\
\hline 25 & $\begin{array}{c}1 \mathrm{H} \\
4.77(\mathrm{~d}) \\
{ }^{3} J_{\mathrm{H} 25-\mathrm{H} 26}=10.6\end{array}$ & $\begin{array}{c}1 \mathrm{H} \\
5.13(\mathrm{~d}) \\
{ }^{3} J_{\mathrm{H} 25-\mathrm{H} 26} \\
=10.8\end{array}$ & $\begin{array}{c}1 \mathrm{H} \\
5.22(\mathrm{~m})\end{array}$ & $\begin{array}{c}1 \mathrm{H} \\
4.91(\mathrm{~d}) \\
{ }^{3} J_{\mathrm{H} 25-\mathrm{H} 26} \\
=10.5\end{array}$ & $\begin{array}{c}1 \mathrm{H} \\
5.32(\mathrm{bs})\end{array}$ & $\begin{array}{c}1 \mathrm{H} \\
5.04(\mathrm{~m})\end{array}$ & $\begin{array}{c}1 \mathrm{H} \\
5.30(\mathrm{bs})\end{array}$ & $\begin{array}{c}1 \mathrm{H} \\
5.16(\mathrm{~d}) \\
{ }^{3} J_{\mathrm{H} 25-\mathrm{H} 26} \\
=10.4\end{array}$ & $\begin{array}{c}1 \mathrm{H} \\
5.28(\mathrm{~m})\end{array}$ \\
\hline 26 & $\begin{array}{c}1 \mathrm{H} \\
1.76(\mathrm{~m}) \\
\end{array}$ & $\begin{array}{c}1 \mathrm{H} \\
0.86(\mathrm{~m}) \\
\end{array}$ & $\begin{array}{c}1 \mathrm{H} \\
2.08(\mathrm{~m}) \\
\end{array}$ & $\begin{array}{c}1 \mathrm{H} \\
0.95(\mathrm{~m}) \\
\end{array}$ & NF & $\begin{array}{c}1 \mathrm{H} \\
1.25(\mathrm{~m})\end{array}$ & $\begin{array}{c}1 \mathrm{H} \\
1.94(\mathrm{~m})\end{array}$ & $\begin{array}{c}1 \mathrm{H} \\
0.90(\mathrm{~m}) \\
\end{array}$ & $\begin{array}{c}1 \mathrm{H} \\
2.01(\mathrm{~m}) \\
\end{array}$ \\
\hline
\end{tabular}




\begin{tabular}{|c|c|c|c|c|c|c|c|c|c|}
\hline $\begin{array}{l}\text { Proton } \\
\text { number }\end{array}$ & $\begin{array}{c}\mathbf{R F B}(\mathbf{A}) \\
\mathrm{CDCl}_{3}\end{array}$ & $\begin{array}{c}\text { RFB(A) } \\
\text { DMSO-d }_{6}\end{array}$ & $\begin{array}{c}\mathbf{R F B}(\mathbf{C}) \\
\text { DMSO-d }_{6}\end{array}$ & $\begin{array}{l}\mathbf{R F B}(\mathbf{A}) \\
\mathrm{CD}_{3} \mathrm{CN}\end{array}$ & $\begin{array}{l}\mathbf{R F B}(\mathbf{C}) \\
\mathrm{CD}_{3} \mathrm{CN}\end{array}$ & $\begin{array}{c}\mathbf{R F B}(\mathbf{A}) \\
\mathrm{CD}_{3} \mathrm{CN} \\
-20{ }^{\circ} \mathrm{C} \\
\end{array}$ & $\begin{array}{c}\mathbf{R F B}(\mathbf{C}) \\
\mathrm{CD}_{3} \mathrm{CN} \\
-20^{\circ} \mathrm{C} \\
\end{array}$ & $\begin{array}{c}\mathbf{R F B ( A )} \\
\mathrm{CD}_{3} \mathrm{CN}: \\
\mathrm{DMSO}^{-\mathrm{d}_{6}}\end{array}$ & $\begin{array}{c}\mathbf{R F B}(\mathbf{C}) \\
\mathrm{CD}_{3} \mathrm{CN}: \\
\mathrm{DMSO}^{-\mathrm{d}_{6}}\end{array}$ \\
\hline 27 & $\begin{array}{c}1 \mathrm{H} \\
3.34(\mathrm{~d}) \\
{ }^{3} J_{\mathrm{H} 27-\mathrm{H} 22}=7.3 \\
{ }^{3} J_{\mathrm{H} 26-\mathrm{H} 27}=2.0\end{array}$ & $\begin{array}{c}1 \mathrm{H} \\
3.29(\mathrm{~d}) \\
{ }^{3} J_{\mathrm{H} 27-\mathrm{H} 28}=6.5\end{array}$ & $\begin{array}{c}1 \mathrm{H} \\
3.31(\mathrm{~m})\end{array}$ & $\begin{array}{c}1 \mathrm{H} \\
3.29(\mathrm{bs}) \\
{ }^{3} \mathrm{~J}_{\mathrm{H} 27-\mathrm{H} 28}=6.5\end{array}$ & $\begin{array}{c}1 \mathrm{H} \\
2.96(\mathrm{~m})\end{array}$ & $\begin{array}{c}1 \mathrm{H} \\
3.35(\mathrm{~m})\end{array}$ & $\begin{array}{c}1 \mathrm{H} \\
2.93(\mathrm{~m})\end{array}$ & $\begin{array}{c}1 \mathrm{H} \\
3.33(\mathrm{~m})\end{array}$ & $\begin{array}{c}1 \mathrm{H} \\
3.33(\mathrm{~m})\end{array}$ \\
\hline 28 & $\begin{array}{c}1 \mathrm{H} \\
5.15(\mathrm{dd}) \\
{ }^{3} \mathrm{~J}_{\mathrm{H} 28-\mathrm{H} 29}=12.5\end{array}$ & $\begin{array}{c}1 \mathrm{H} \\
4.86 \\
(\mathrm{dd}) \\
3 J_{\mathrm{H} 28-\mathrm{H} 29} \\
=12.7 \\
{ }^{3} J_{\mathrm{H} 22-\mathrm{H} 228}=6.5\end{array}$ & $\begin{array}{c}1 \mathrm{H} \\
4.82 \\
(\mathrm{dd}) \\
{ }^{3} J_{\mathrm{H} 28-\mathrm{H} 29} \\
=11.5 \\
{ }^{3} J_{\mathrm{H} 27-\mathrm{H} 28}=8.7\end{array}$ & $\begin{array}{c}1 \mathrm{H} \\
5.03 \\
(\mathrm{dd}) \\
{ }^{3} J_{\mathrm{H} 28-\mathrm{H} 29} \\
=12.6 \\
{ }^{3} J_{\mathrm{H} 22-\mathrm{H} 228}=6.7\end{array}$ & $\begin{array}{c}1 \mathrm{H} \\
4.88 \\
(\mathrm{dd}) \\
{ }^{3} J_{\mathrm{H} 28-\mathrm{H} 29} \\
=12.2 \\
{ }^{3} J_{\mathrm{H} 22-\mathrm{H} 28}=9.8\end{array}$ & $\begin{array}{c}1 \mathrm{H} \\
4.96 \\
(\mathrm{dd}) \\
{ }^{3} J_{\mathrm{H} 28-\mathrm{H} 29} \\
=12.6 \\
{ }^{3} J_{\mathrm{H} 27-\mathrm{H} 28}=5.9\end{array}$ & $\begin{array}{c}1 \mathrm{H} \\
4.82 \\
(\mathrm{~m})\end{array}$ & $\begin{array}{c}1 \mathrm{H} \\
4.90 \\
(\mathrm{dd}) \\
{ }^{3} J_{\mathrm{H} 28-\mathrm{H} 29} \\
=12.7 \\
{ }^{3} J_{\mathrm{H} 27-\mathrm{H} 28}=6.5\end{array}$ & $\begin{array}{c}1 \mathrm{H} \\
4.87 \\
(\mathrm{~m})\end{array}$ \\
\hline 29 & $\begin{array}{c}1 \mathrm{H} \\
6.16(\mathrm{~d})\end{array}$ & $\begin{array}{c}1 \mathrm{H} \\
6.12(\mathrm{~d})\end{array}$ & $\begin{array}{c}1 \mathrm{H} \\
6.14(\mathrm{~d})\end{array}$ & $\begin{array}{c}1 \mathrm{H} \\
6.10(\mathrm{~d})\end{array}$ & $\begin{array}{c}1 \mathrm{H} \\
5.88(\mathrm{~d}) \\
{ }^{3} J_{\mathrm{H} 28-\mathrm{H} 29} \\
=12.0\end{array}$ & $\begin{array}{c}1 \mathrm{H} \\
6.04(\mathrm{~d})\end{array}$ & $\begin{array}{c}1 \mathrm{H} \\
5.83(\mathrm{~d}) \\
{ }^{3} J_{\mathrm{H} 28-\mathrm{H} 29} \\
=12.0\end{array}$ & $\begin{array}{c}1 \mathrm{H} \\
6.12(\mathrm{~d})\end{array}$ & $\begin{array}{c}1 \mathrm{H} \\
6.15(\mathrm{~d}) \\
{ }^{3} J_{\mathrm{H} 28-\mathrm{H} 29} \\
=11.7 \mathrm{j}\end{array}$ \\
\hline 30 & $\begin{array}{c}3 \mathrm{H} \\
2.05(\mathrm{~s})\end{array}$ & $\begin{array}{c}3 \mathrm{H} \\
1.97(\mathrm{~s})\end{array}$ & $\begin{array}{c}3 \mathrm{H} \\
1.93(\mathrm{~s})\end{array}$ & $\begin{array}{c}3 \mathrm{H} \\
2.00(\mathrm{~s})\end{array}$ & $\begin{array}{c}3 \mathrm{H} \\
2.03(\mathrm{~s})\end{array}$ & $\begin{array}{c}3 \mathrm{H} \\
2.00(\mathrm{~s})\end{array}$ & $\begin{array}{c}3 \mathrm{H} \\
2.03(\mathrm{~s})\end{array}$ & $\begin{array}{c}3 \mathrm{H} \\
1.94(\mathrm{~s})\end{array}$ & $\begin{array}{c}3 \mathrm{H} \\
1.96(\mathrm{~s})\end{array}$ \\
\hline 31 & $\begin{array}{c}3 \mathrm{H} \\
0.84(\mathrm{~d}) \\
{ }^{3} \mathrm{~J}_{\mathrm{H} 20 \mathrm{H} 31}=6.9\end{array}$ & $\begin{array}{c}3 \mathrm{H} \\
0.83(\mathrm{~d}) \\
{ }^{3} \mathrm{~J}_{\mathrm{H} 20 \mathrm{-H} 3}=6.9\end{array}$ & $\begin{array}{c}3 \mathrm{H} \\
0.67(\mathrm{~d}) \\
{ }^{3} \mathrm{~J}_{\mathrm{H} 20 \mathrm{H} 31}=6.7\end{array}$ & $\begin{array}{c}3 \mathrm{H} \\
0.83(\mathrm{~d}) \\
{ }^{3} \mathrm{~J}_{\mathrm{H} 20-\mathrm{H} 3 \mathrm{l}}=6.9\end{array}$ & $\begin{array}{c}3 \mathrm{H} \\
0.58(\mathrm{~d}) \\
{ }^{3} \mathrm{~J}_{\mathrm{H} 20-\mathrm{H} 3 \mathrm{l}}=6.7\end{array}$ & $\begin{array}{c}3 \mathrm{H} \\
0.82(\mathrm{~d}) \\
{ }^{3} \mathrm{~J}_{\mathrm{H} 20 \mathrm{H} 33}=6.9\end{array}$ & $\begin{array}{c}3 \mathrm{H} \\
0.47(\mathrm{bs})\end{array}$ & $\begin{array}{c}3 \mathrm{H} \\
0.84(\mathrm{~d}) \\
{ }^{3} \mathrm{~J}_{\mathrm{H} 20 \mathrm{H} 33}=7.1 \mathrm{j}\end{array}$ & $\begin{array}{c}3 \mathrm{H} \\
0.68(\mathrm{~d}) \\
{ }^{3} J_{\mathrm{H} 20-\mathrm{H} 31}=6.7\end{array}$ \\
\hline 32 & $\begin{array}{c}3 \mathrm{H} \\
1.04(\mathrm{~d}) \\
{ }^{3} \mathrm{H}_{\mathrm{H} 22-\mathrm{H} 32}=7.0\end{array}$ & $\begin{array}{c}3 \mathrm{H} \\
0.92(\mathrm{~d}) \\
{ }^{3} J_{\mathrm{H} 22-\mathrm{H} 32}=6.5\end{array}$ & $\begin{array}{c}3 \mathrm{H} \\
0.86(\mathrm{~d}) \\
{ }^{3} \mathrm{~J}_{\mathrm{H} 22-\mathrm{H} 32}=7.0\end{array}$ & $\begin{array}{c}3 \mathrm{H} \\
0.95(\mathrm{~d}) \\
{ }^{3} J_{\mathrm{H} 22-\mathrm{H} 32}=6.5\end{array}$ & $\begin{array}{c}3 \mathrm{H} \\
0.86(\mathrm{~d}) \\
{ }^{3} J_{\mathrm{H} 22-\mathrm{H} 32}=7.2\end{array}$ & $\begin{array}{c}3 \mathrm{H} \\
0.95(\mathrm{~m})\end{array}$ & $\begin{array}{c}3 \mathrm{H} \\
0.78(\mathrm{~d}) \\
{ }^{3} \mathrm{~J}_{\mathrm{H} 22-\mathrm{H} 32}=6.9\end{array}$ & $\begin{array}{c}3 \mathrm{H} \\
0.95(\mathrm{~d}) \\
{ }^{3} \mathrm{~J}_{\mathrm{H} 22-\mathrm{H} 32}=7.1\end{array}$ & $\begin{array}{c}3 \mathrm{H} \\
0.89(\mathrm{~d}) \\
{ }^{3} J_{\mathrm{H} 22-\mathrm{H} 32}=7.1\end{array}$ \\
\hline 33 & $\begin{array}{c}3 \mathrm{H} \\
0.60(\mathrm{~d}) \\
{ }^{3} J_{\mathrm{H} 24-\mathrm{H} 33}=6.9\end{array}$ & $\begin{array}{c}3 \mathrm{H} \\
0.41(\mathrm{~d}) \\
{ }^{3} J_{\mathrm{H} 24-\mathrm{H} 33}=6.8\end{array}$ & $\begin{array}{c}3 \mathrm{H} \\
0.70(\mathrm{~d}) \\
{ }^{3} J_{\mathrm{H} 24-\mathrm{H} 33}=6.5\end{array}$ & $\begin{array}{c}3 \mathrm{H} \\
0.52(\mathrm{~d}) \\
{ }^{3} J_{\mathrm{H} 24-\mathrm{H} 3}=6.9\end{array}$ & $\begin{array}{c}3 \mathrm{H} \\
0.74(\mathrm{~d}) \\
{ }^{3} J_{\mathrm{H} 24-\mathrm{H} 33}=6.6\end{array}$ & $\begin{array}{c}3 \mathrm{H} \\
0.51(\mathrm{~d}) \\
{ }^{3} J_{\mathrm{H} 24-\mathrm{H} 33}=6.8\end{array}$ & $\begin{array}{c}3 \mathrm{H} \\
0.70(\mathrm{~d}) \\
{ }^{3} J_{\mathrm{H} 24-\mathrm{H} 33}=6.4\end{array}$ & $\begin{array}{c}3 \mathrm{H} \\
0.46(\mathrm{~d}) \\
{ }^{3} J_{\mathrm{H} 24-\mathrm{H} 33}=7.1\end{array}$ & $\begin{array}{c}3 \mathrm{H} \\
0.74(\mathrm{~d}) \\
{ }^{3} J_{\mathrm{H} 24-\mathrm{H} 33}=6.7\end{array}$ \\
\hline 34 & $\begin{array}{c}3 \mathrm{H} \\
-0.07(\mathrm{~d}) \\
3_{\mathrm{H} 26-\mathrm{H} 36}=7.1\end{array}$ & $\begin{array}{c}3 \mathrm{H} \\
-0.24(\mathrm{~d}) \\
{ }^{3} J_{\mathrm{H} 34-\mathrm{H} 26}=6.9\end{array}$ & $\begin{array}{c}3 \mathrm{H} \\
0.90(\mathrm{~d}) \\
{ }^{3} J_{\mathrm{H} 34-\mathrm{H} 26}=6.4\end{array}$ & $\begin{array}{c}3 \mathrm{H} \\
-0.16(\mathrm{~d}) \\
{ }^{3} J_{\mathrm{H} 34-\mathrm{H} 26}=7.1\end{array}$ & $\begin{array}{c}3 \mathrm{H} \\
0.93(\mathrm{~m})\end{array}$ & $\begin{array}{c}3 \mathrm{H} \\
-0.22(\mathrm{~d}) \\
3_{\mathrm{H} 34-\mathrm{H} 26}=7.0\end{array}$ & $\begin{array}{c}3 \mathrm{H} \\
0.95(\mathrm{~m})\end{array}$ & $\begin{array}{c}3 \mathrm{H} \\
-0.21(\mathrm{~d}) \\
3_{\mathrm{H} 34-\mathrm{H} 26}=7.1\end{array}$ & $\begin{array}{c}3 \mathrm{H} \\
0.92(\mathrm{~d}) \\
{ }^{3} J_{\mathrm{H} 26-\mathrm{H} 34}=6.7\end{array}$ \\
\hline 35 & --- & --- & --- & --- & --- & --- & --- & --- & --- \\
\hline 36 & $\begin{array}{c}3 \mathrm{H} \\
2.01(\mathrm{~s})\end{array}$ & $\begin{array}{c}3 \mathrm{H} \\
1.95(\mathrm{~s})\end{array}$ & $\begin{array}{c}3 \mathrm{H} \\
1.96(\mathrm{~s})\end{array}$ & $\begin{array}{c}3 \mathrm{H} \\
1.93(\mathrm{~s})\end{array}$ & $\begin{array}{c}3 \mathrm{H} \\
1.96(\mathrm{~s})\end{array}$ & $\begin{array}{c}3 \mathrm{H} \\
1.94(\mathrm{~s})\end{array}$ & $\begin{array}{c}3 \mathrm{H} \\
1.94(\mathrm{~s})\end{array}$ & $\begin{array}{c}3 \mathrm{H} \\
1.98(\mathrm{~s})\end{array}$ & $\begin{array}{c}3 \mathrm{H} \\
1.96(\mathrm{~s})\end{array}$ \\
\hline
\end{tabular}




\begin{tabular}{|c|c|c|c|c|c|c|c|c|c|}
\hline $\begin{array}{l}\text { Proton } \\
\text { number }\end{array}$ & $\begin{array}{c}\mathbf{R F B}(\mathbf{A}) \\
\mathrm{CDCl}_{3}\end{array}$ & $\begin{array}{c}\text { RFB(A) } \\
\text { DMSO-d }_{6}\end{array}$ & $\begin{array}{c}\mathbf{R F B}(\mathbf{C}) \\
\text { DMSO-d }_{6}\end{array}$ & $\begin{array}{l}\mathbf{R F B}(\mathbf{A}) \\
\mathrm{CD}_{3} \mathrm{CN}\end{array}$ & $\begin{array}{l}\mathbf{R F B}(\mathbf{C}) \\
\mathrm{CD}_{3} \mathrm{CN}\end{array}$ & $\begin{array}{c}\mathbf{R F B}(\mathbf{A}) \\
\mathrm{CD}_{3} \mathrm{CN} \\
-20{ }^{\circ} \mathrm{C} \\
\end{array}$ & $\begin{array}{c}\mathbf{R F B}(\mathbf{C}) \\
\mathrm{CD}_{3} \mathrm{CN} \\
-20{ }^{\circ} \mathrm{C} \\
\end{array}$ & $\begin{array}{c}\mathbf{R F B ( A )} \\
\mathrm{CD}_{3} \mathrm{CN}: \\
\mathrm{DMSO}^{-\mathrm{d}_{6}}\end{array}$ & $\begin{array}{c}\mathbf{R F B}(\mathbf{C}) \\
\mathrm{CD}_{3} \mathrm{CN}: \\
\mathrm{DMSO}^{-\mathrm{d}_{6}}\end{array}$ \\
\hline 37 & $\begin{array}{c}3 \mathrm{H} \\
3.08(\mathrm{~s})\end{array}$ & $\begin{array}{c}3 \mathrm{H} \\
2.91(\mathrm{~s})\end{array}$ & $\begin{array}{c}3 \mathrm{H} \\
3.08(\mathrm{~s})\end{array}$ & $\begin{array}{c}3 \mathrm{H} \\
3.00(\mathrm{~s})\end{array}$ & $\begin{array}{c}3 \mathrm{H} \\
3.05(\mathrm{~s})\end{array}$ & $\begin{array}{c}3 \mathrm{H} \\
2.97(\mathrm{~s})\end{array}$ & $\begin{array}{c}3 \mathrm{H} \\
3.01(\mathrm{~s})\end{array}$ & $\begin{array}{c}3 \mathrm{H} \\
2.95(\mathrm{~s})\end{array}$ & $\begin{array}{c}3 \mathrm{H} \\
3.10(\mathrm{~s})\end{array}$ \\
\hline 38 & -- & -- & -- & -- & -- & -- & -- & -- & -- \\
\hline 39 & $\begin{array}{c}1 \mathrm{H} \\
1.96(\mathrm{~m}) \\
1 \mathrm{H} \\
2.14(\mathrm{~m})\end{array}$ & $\begin{array}{c}1 \mathrm{H} \\
2.32(\mathrm{~m}) \\
1 \mathrm{H} \\
1.57(\mathrm{~m})\end{array}$ & $\begin{array}{c}1 \mathrm{H} \\
1.41(\mathrm{~m}) \\
1 \mathrm{H} \\
1.96(\mathrm{~m})\end{array}$ & $\begin{array}{c}1 \mathrm{H} \\
2.30(\mathrm{~m}) \\
1 \mathrm{H} \\
1.38(\mathrm{~m})\end{array}$ & $\mathrm{NF}$ & $\mathrm{NF}$ & NF & $\begin{array}{c}1 \mathrm{H} \\
2.30(\mathrm{~m}) \\
1 \mathrm{H} \\
1.58(\mathrm{~m})\end{array}$ & $\begin{array}{c}1 \mathrm{H} \\
1.49(\mathrm{~m}) \\
1 \mathrm{H} \\
1.98(\mathrm{~m})\end{array}$ \\
\hline 40 & $\begin{array}{c}1 \mathrm{H} \\
2.96(\mathrm{~m}) \\
1 \mathrm{H} \\
2.63(\mathrm{~m})\end{array}$ & $\begin{array}{c}1 \mathrm{H} \\
2.59(\mathrm{~m}) \\
1 \mathrm{H} \\
2.78(\mathrm{~m})\end{array}$ & $\begin{array}{c}1 \mathrm{H} \\
2.63(\mathrm{~m}) \\
1 \mathrm{H} \\
2.87(\mathrm{~m})\end{array}$ & $\begin{array}{c}4 \mathrm{H} \\
2.76-2.66 \\
(\mathrm{~m})\end{array}$ & $\mathrm{NF}$ & NF & $\mathrm{NF}$ & $\begin{array}{c}1 \mathrm{H} \\
2.61(\mathrm{~m}) \\
1 \mathrm{H} \\
2.78(\mathrm{~m})\end{array}$ & $\begin{array}{c}1 \mathrm{H} \\
2.65(\mathrm{~m}) \\
1 \mathrm{H} \\
2.88(\mathrm{~m})\end{array}$ \\
\hline 41 & $\begin{array}{c}1 \mathrm{H} \\
2.96(\mathrm{~m}) \\
1 \mathrm{H} \\
2.63(\mathrm{~m})\end{array}$ & $\begin{array}{c}1 \mathrm{H} \\
2.59(\mathrm{~m}) \\
1 \mathrm{H} \\
2.78(\mathrm{~m})\end{array}$ & $\begin{array}{c}1 \mathrm{H} \\
2.63(\mathrm{~m}) \\
1 \mathrm{H} \\
2.87(\mathrm{~m})\end{array}$ & & $\mathrm{NF}$ & $\mathrm{NF}$ & $\mathrm{NF}$ & $\begin{array}{c}1 \mathrm{H} \\
2.61(\mathrm{~m}) \\
1 \mathrm{H} \\
2.78(\mathrm{~m})\end{array}$ & $\begin{array}{c}1 \mathrm{H} \\
2.65(\mathrm{~m}) \\
1 \mathrm{H} \\
2.88(\mathrm{~m})\end{array}$ \\
\hline 42 & $\begin{array}{c}1 \mathrm{H} \\
1.83(\mathrm{~m}) \\
1 \mathrm{H} \\
1.96(\mathrm{~m})\end{array}$ & $\begin{array}{c}1 \mathrm{H} \\
2.32(\mathrm{~m}) \\
1 \mathrm{H} \\
1.57(\mathrm{~m})\end{array}$ & $\begin{array}{c}1 \mathrm{H} \\
2.32(\mathrm{~m}) \\
1 \mathrm{H} \\
1.21(\mathrm{~m})\end{array}$ & $\begin{array}{c}1 \mathrm{H} \\
2.30(\mathrm{~m}) \\
1 \mathrm{H} \\
1.38(\mathrm{~m})\end{array}$ & $\mathrm{NF}$ & NF & NF & $\begin{array}{c}1 \mathrm{H} \\
2.30(\mathrm{~m}) \\
1 \mathrm{H} \\
1.58(\mathrm{~m})\end{array}$ & $\begin{array}{c}1 \mathrm{H} \\
2.30(\mathrm{~m}) \\
1 \mathrm{H} \\
1.29(\mathrm{~m})\end{array}$ \\
\hline 43 & $\begin{array}{c}2 \mathrm{H} \\
2.28(\mathrm{~d}) \\
3_{\mathrm{H} 43-\mathrm{H} 44}=7.3\end{array}$ & $\begin{array}{c}2 \mathrm{H} \\
2.25(\mathrm{~m})\end{array}$ & $\begin{array}{c}2 \mathrm{H} \\
2.21(\mathrm{~m})\end{array}$ & $\begin{array}{c}2 \mathrm{H} \\
2.25(\mathrm{~m})\end{array}$ & $\begin{array}{c}2 \mathrm{H} \\
2.22(\mathrm{~m})\end{array}$ & $\begin{array}{c}2 \mathrm{H} \\
2.25(\mathrm{~m})\end{array}$ & $\begin{array}{c}2 \mathrm{H} \\
2.22(\mathrm{~m})\end{array}$ & $\begin{array}{c}2 \mathrm{H} \\
2.26(\mathrm{~m})\end{array}$ & $\begin{array}{c}2 \mathrm{H} \\
2.23(\mathrm{~m})\end{array}$ \\
\hline 44 & $\begin{array}{c}1 \mathrm{H} \\
1.82(\mathrm{~m})\end{array}$ & $\begin{array}{c}1 \mathrm{H} \\
1.85(\mathrm{~m})\end{array}$ & $\begin{array}{c}1 \mathrm{H} \\
1.81(\mathrm{~m})\end{array}$ & $\begin{array}{c}1 \mathrm{H} \\
1.85(\mathrm{~m})\end{array}$ & $\begin{array}{c}1 \mathrm{H} \\
1.82(\mathrm{~m})\end{array}$ & $\begin{array}{c}1 \mathrm{H} \\
1.86(\mathrm{~m})\end{array}$ & $\begin{array}{c}1 \mathrm{H} \\
1.85(\mathrm{~m})\end{array}$ & $\begin{array}{c}1 \mathrm{H} \\
1.87(\mathrm{~m})\end{array}$ & $\begin{array}{c}1 \mathrm{H} \\
1.83(\mathrm{~m})\end{array}$ \\
\hline
\end{tabular}




\begin{tabular}{|c|c|c|c|c|c|c|c|c|c|}
\hline $\begin{array}{l}\text { Proton } \\
\text { number }\end{array}$ & $\underset{\mathrm{CDCl}_{3}}{\mathbf{R F B ( A )}}$ & $\begin{array}{c}\text { RFB(A) } \\
\text { DMSO-d }_{6}\end{array}$ & $\begin{array}{c}\mathbf{R F B}(\mathbf{C}) \\
\text { DMSO-d }_{6}\end{array}$ & $\begin{array}{l}\mathbf{R F B}(\mathbf{A}) \\
\mathrm{CD}_{3} \mathrm{CN}\end{array}$ & $\begin{array}{l}\mathbf{R F B}(\mathbf{C}) \\
\mathrm{CD}_{3} \mathrm{CN}\end{array}$ & $\begin{array}{c}\mathbf{R F B}(\mathbf{A}) \\
\mathrm{CD}_{3} \mathrm{CN} \\
-20{ }^{\circ} \mathrm{C} \\
\end{array}$ & $\begin{array}{c}\mathbf{R F B}(\mathbf{C}) \\
\mathrm{CD}_{3} \mathrm{CN} \\
-20^{\circ} \mathrm{C} \\
\end{array}$ & $\begin{array}{c}\text { RFB(A) } \\
\text { CD }_{3} \text { CN: } \\
\text { DMSO-d }_{6}\end{array}$ & $\begin{array}{c}\mathbf{R F B}(\mathbf{C}) \\
\mathrm{CD}_{3} \mathrm{CN}: \\
\mathrm{DMSO}^{-d_{6}}\end{array}$ \\
\hline $45+46$ & $\begin{array}{c}6 \mathrm{H} \\
0.90(\mathrm{~d}) \\
{ }^{3} J_{\mathrm{H} 45.46-\mathrm{H} 4}=6.5\end{array}$ & $\begin{array}{c}6 \mathrm{H} \\
0.91(\mathrm{~d}) \\
{ }_{3} J_{\mathrm{H} 45.46-\mathrm{H} 4}=6.5\end{array}$ & $\begin{array}{c}6 \mathrm{H} \\
0.90(\mathrm{~d}) \\
{ }^{3} J_{\mathrm{H} 45}-46-\mathrm{H} 44=6.5\end{array}$ & $\begin{array}{c}6 \mathrm{H} \\
0.94(\mathrm{~d}) \\
{ }^{3} J_{\mathrm{H} 45.46-\mathrm{H} 44}=6.5\end{array}$ & 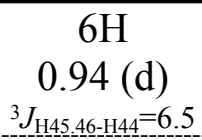 & $\begin{array}{c}6 \mathrm{H} \\
0.93(\mathrm{~m})\end{array}$ & $\begin{array}{c}6 \mathrm{H} \\
0.93(\mathrm{~m})\end{array}$ & $\begin{array}{c}6 \mathrm{H} \\
0.93(\mathrm{~d}) \\
{ }_{3} J_{\mathrm{H}} 4546-\mathrm{H}-\mathrm{H} 4=6.7\end{array}$ & $\begin{array}{c}6 \mathrm{H} \\
0.93(\mathrm{~d}) \\
{ }^{3} J_{\mathrm{H}}-9546-\mathrm{H}-\mathrm{H} 4=6.7\end{array}$ \\
\hline $\mathrm{OH}-1$ & -- & -- & --- & --- & -- & -- & --- & -- & -- \\
\hline $\mathrm{OH}-4$ & -- & -- & -- & -- & -- & -- & -- & -- & -- \\
\hline $\mathrm{OH}-8$ & $\begin{array}{c}1 \mathrm{H} \\
14.73(\mathrm{~s})\end{array}$ & $\begin{array}{c}1 \mathrm{H} \\
16.16(\mathrm{~s})\end{array}$ & $\begin{array}{c}1 \mathrm{H} \\
16.23(\mathrm{~s})\end{array}$ & $\begin{array}{c}1 \mathrm{H} \\
15.22(\mathrm{~s})\end{array}$ & $\begin{array}{c}1 \mathrm{H} \\
15.66(\mathrm{~s})\end{array}$ & $\begin{array}{c}1 \mathrm{H} \\
15.45(\mathrm{~s})\end{array}$ & $\begin{array}{c}1 \mathrm{H} \\
15.68(\mathrm{~s})\end{array}$ & $\begin{array}{c}1 \mathrm{H} \\
16.04(\mathrm{~s})\end{array}$ & $\begin{array}{c}1 \mathrm{H} \\
16.18(\mathrm{~s})\end{array}$ \\
\hline $\mathrm{OH}-21$ & $\begin{array}{c}1 \mathrm{H} \\
3.43(\mathrm{~s})\end{array}$ & $\begin{array}{c}1 \mathrm{H} \\
4.95(\mathrm{~d}) \\
{ }^{3} J_{\mathrm{HO}-\mathrm{H} 21}=3.3\end{array}$ & $\begin{array}{c}1 \mathrm{H} \\
4.39(\mathrm{~d}) \\
{ }^{3} J_{\mathrm{HO}-\mathrm{H} 21}=3.1\end{array}$ & $\begin{array}{c}1 \mathrm{H} \\
5.10(\mathrm{vbs})\end{array}$ & $\mathrm{NF}$ & $\begin{array}{c}1 \mathrm{H} \\
5.02(\mathrm{~m})\end{array}$ & $\mathrm{NF}$ & $\begin{array}{c}1 \mathrm{H} \\
4.90(\mathrm{~m})\end{array}$ & $\begin{array}{c}1 \mathrm{H} \\
4.11(\mathrm{~m})\end{array}$ \\
\hline $\mathrm{OH}-23$ & $\begin{array}{c}1 \mathrm{H} \\
3.63(\mathrm{~d})\end{array}$ & $\begin{array}{c}1 \mathrm{H} \\
4.16(\mathrm{~d}) \\
{ }^{3} J_{\text {브-플 } 3}=8.4\end{array}$ & $\begin{array}{c}1 \mathrm{H} \\
3.61(\mathrm{~d}) \\
{ }^{3} J_{\text {브-는 } 3=9.6}\end{array}$ & $\begin{array}{c}1 \mathrm{H} \\
3.36(\mathrm{~d}) \\
{ }^{3} J_{\mathrm{HO}-\underline{-1} 23}=5.6\end{array}$ & $\begin{array}{c}1 \mathrm{H} \\
3.18(\mathrm{~m})\end{array}$ & $\begin{array}{c}1 \mathrm{H} \\
3.86(\mathrm{bs})\end{array}$ & $\begin{array}{c}1 \mathrm{H} \\
3.24(\mathrm{bs})\end{array}$ & $\begin{array}{c}1 \mathrm{H} \\
4.13(\mathrm{~d}) \\
{ }^{3} J_{\mathrm{HO}-\mathrm{H} 23}=8.8\end{array}$ & $\begin{array}{c}1 \mathrm{H} \\
3.66(\mathrm{~d}) \\
{ }^{3} J_{\mathrm{HO}-\mathrm{H} 23}=10.0\end{array}$ \\
\hline NH (amid) & $\begin{array}{c}1 \mathrm{H} \\
8.19(\mathrm{~s})\end{array}$ & $\begin{array}{c}1 \mathrm{H} \\
8.92(\mathrm{~s})\end{array}$ & $\begin{array}{c}1 \mathrm{H} \\
8.92(\mathrm{~s})\end{array}$ & $\begin{array}{c}1 \mathrm{H} \\
8.06(\mathrm{~s})\end{array}$ & $\begin{array}{c}1 \mathrm{H} \\
8.11(\mathrm{~s})\end{array}$ & $\begin{array}{c}1 \mathrm{H} \\
8.57(\mathrm{~s})\end{array}$ & $\begin{array}{c}1 \mathrm{H} \\
8.47(\mathrm{~s})\end{array}$ & $\begin{array}{c}1 \mathrm{H} \\
8.91(\mathrm{~s})\end{array}$ & $\begin{array}{c}1 \mathrm{H} \\
8.69(\mathrm{~s})\end{array}$ \\
\hline $\mathrm{N}^{+} \mathrm{H}$ & -- & -- & -- & -- & -- & -- & -- & -- & -- \\
\hline $\mathrm{N}(3)-\mathrm{H}$ & $\begin{array}{c}1 \mathrm{H} \\
9.10(\mathrm{~s})\end{array}$ & $\begin{array}{c}1 \mathrm{H} \\
10.22(\mathrm{~s})\end{array}$ & $\begin{array}{c}1 \mathrm{H} \\
10.67(\mathrm{~s})\end{array}$ & $\begin{array}{c}1 \mathrm{H} \\
8.74(\mathrm{~s}) \\
\end{array}$ & $\begin{array}{c}1 \mathrm{H} \\
8.74(\mathrm{~s})\end{array}$ & $\begin{array}{c}1 \mathrm{H} \\
8.92(\mathrm{~s})\end{array}$ & $\begin{array}{c}1 \mathrm{H} \\
8.92(\mathrm{~s}) \\
\end{array}$ & $\begin{array}{c}1 \mathrm{H} \\
9.89(\mathrm{~s})\end{array}$ & $\begin{array}{c}1 \mathrm{H} \\
10.56(\mathrm{~s}) \\
\end{array}$ \\
\hline
\end{tabular}

NF- signal not found 


\section{Table S4}

${ }^{13} \mathrm{C}$ NMR data of Rifabutin (RFB) assigned on the basis of ${ }^{1} \mathrm{H}-{ }^{13} \mathrm{C}$ HSQC, ${ }^{1} \mathrm{H}-{ }^{13} \mathrm{C}$ HMBC, ${ }^{1} \mathrm{H}-{ }^{1} \mathrm{H}$ COSY and ${ }^{1} \mathrm{H}-{ }^{1} \mathrm{H}$ NOESY correlation spectra.

\begin{tabular}{|c|c|c|c|c|c|}
\hline $\begin{array}{l}\text { Numer } \\
\text { atomu } \\
\text { węgla }\end{array}$ & $\begin{array}{c}\mathbf{R F B}(\mathbf{A}) \\
\mathrm{CDCl}_{3}\end{array}$ & $\begin{array}{c}\mathbf{R F B ( A )} \\
\text { DMSO-d }_{6}\end{array}$ & $\begin{array}{c}\mathbf{R F B}(\mathbf{C}) \\
\text { DMSO-d }_{6}\end{array}$ & $\begin{array}{l}\mathbf{R F B}(\mathbf{A}) \\
\mathrm{CD}_{3} \mathrm{CN}\end{array}$ & $\begin{array}{l}\mathbf{R F B}(\mathbf{C}) \\
\mathrm{CD}_{3} \mathrm{CN}\end{array}$ \\
\hline 1 & 181.0 & 182.1 & 183.5 & 182.7 & $\mathrm{NF}$ \\
\hline 2 & 111.7 & 112.2 & 112.4 & 112.9 & 113.5 \\
\hline 3 & 142.0 & 145.9 & 148.5 & 144.6 & NF \\
\hline 4 & 155.1 & 154.9 & 155.8 & 156.0 & 156.8 \\
\hline 5 & 108.9 & 108.5 & 106.9 & 109.8 & 106.8 \\
\hline 6 & 171.5 & 170.8 & 170.4 & 172.1 & 172.0 \\
\hline 7 & 114.3 & 112.3 & 113.7 & 114.3 & 114.6 \\
\hline 8 & 168.2 & 168.0 & 169.2 & 168.6 & 168.7 \\
\hline 9 & 104.5 & 101.7 & 102.6 & 104.6 & 104.2 \\
\hline 10 & 125.1 & 124.8 & 124.7 & 126.4 & 126.5 \\
\hline 11 & 192.5 & 191.2 & 191.8 & 192.9 & 193.0 \\
\hline 12 & 107.2 & 106.6 & 105.3 & 107.8 & 106.9 \\
\hline 13 & 21.9 & 21.2 & 22.2 & 21.8 & 22.5 \\
\hline 14 & 7.6 & 7.5 & 7.6 & 7.8 & 8.1 \\
\hline 15 & 168.5 & 166.4 & 171.4 & 169.0 & 171.3 \\
\hline 16 & 131.3 & 131.0 & 128.5 & 132.6 & 128.2 \\
\hline 17 & 133.0 & 132.2 & 129.4 & 133.1 & 131.8 \\
\hline 18 & 124.0 & 127.2 & 126.5 & 125.9 & NF \\
\hline 19 & 141.0 & 138.4 & 140.8 & 141.3 & 142.2 \\
\hline 20 & 38.2 & 37.5 & 41.7 & 39.3 & 43.3 \\
\hline 21 & 72.7 & 72.9 & 72.2 & 73.7 & NF \\
\hline 22 & 33.1 & 32.4 & 31.1 & 33.9 & 32.2 \\
\hline 23 & 76.9 & 75.9 & 77.1 & 77.5 & 78.8 \\
\hline
\end{tabular}




\begin{tabular}{|c|c|c|c|c|c|}
\hline $\begin{array}{l}\text { Numer } \\
\text { atomu } \\
\text { węgla }\end{array}$ & $\begin{array}{c}\mathbf{R F B ( A )} \\
\mathrm{CDCl}_{3}\end{array}$ & $\begin{array}{c}\mathbf{R F B ( A )} \\
\text { DMSO-d }_{6}\end{array}$ & $\begin{array}{c}\mathbf{R F B}(\mathbf{C}) \\
\mathrm{DMSO}^{-\mathrm{d}_{6}}\end{array}$ & $\begin{array}{c}\mathbf{R F B}(\mathbf{A}) \\
\mathrm{CD}_{3} \mathrm{CN}\end{array}$ & $\begin{array}{c}\mathbf{R F B}(\mathbf{C}) \\
\mathrm{CD}_{3} \mathrm{CN}\end{array}$ \\
\hline 24 & 37.6 & 38.0 & 35.5 & 39.4 & NF \\
\hline 25 & 73.2 & 72.5 & 72.9 & 73.9 & 74.1 \\
\hline 26 & 37.7 & 39.5 & 41.3 & 38.7 & 43.0 \\
\hline 27 & 80.7 & 75.8 & 77.6 & 79.4 & 80.2 \\
\hline 28 & 115.8 & 117.8 & 110.2 & 118.0 & 111.3 \\
\hline 29 & 144.2 & 142.0 & 143.9 & 143.7 & NF \\
\hline 30 & 20.2 & 20.2 & 20.5 & 20.6 & 21.1 \\
\hline 31 & 17.4 & 17.9 & 16.5 & 18.0 & 16.5 \\
\hline 32 & 11.2 & 11.0 & 10.7 & 11.4 & 11.1 \\
\hline 33 & 8.7 & 9.1 & 12.1 & 9.3 & 12.5 \\
\hline 34 & 10.9 & 8.4 & 12.3 & 10.4 & 12.7 \\
\hline 35 & 172.1 & 169.3 & 169.8 & 172.3 & NF \\
\hline 36 & 21.0 & 20.6 & 20.6 & 21.2 & 21.2 \\
\hline 37 & 56.8 & 56.1 & 54.1 & 57.1 & 55.2 \\
\hline 38 & 94.7 & 94.8 & 94.4 & 96.9 & 96.9 \\
\hline 39 & 35.4 & 34.9 & 34.7 & 36.0 & 35.6 \\
\hline 40 & 51.5 & 51.0 & 50.6 & 52.3 & 51.6 \\
\hline 41 & 51.4 & 50.7 & 50.5 & 52.1 & 51.6 \\
\hline 42 & 36.3 & 35.0 & 34.8 & 36.7 & 36.8 \\
\hline 43 & 66.3 & 65.6 & 65.8 & 67.1 & 67.0 \\
\hline 44 & 25.9 & 25.3 & 25.2 & 26.6 & 26.6 \\
\hline 45 & 20.8 & \multirow[t]{2}{*}{20.8} & \multirow[t]{2}{*}{20.7} & \multirow[t]{2}{*}{21.1} & \multirow[t]{2}{*}{21.1} \\
\hline 46 & 20.9 & & & & \\
\hline
\end{tabular}

NF- signal not found 


\section{Table S5}

Calculated values of $\Delta \mathrm{H}$ for RFB from the van't Hoff equation.

\begin{tabular}{llll}
\hline & \multicolumn{1}{c}{$\Delta \mathrm{H}(\mathrm{kcal})$} & \\
& for methyl signals & for phenol signals & \multicolumn{1}{c}{ for amine signals } \\
$\mathrm{DMSO}^{-} \mathrm{d}_{6}^{*}$ & $3.45 \pm 0.39$ & -- & $4.54 \pm 0.22$ \\
$\mathrm{CD}_{3} \mathrm{CN}^{* *}$ & $6.15 \pm 0.42$ & $6.50 \pm 0.81$ & --- \\
pyridine-d $_{5}^{* * *}$ & $5.03 \pm 0.96$ & $5.00 \pm 0.53$ & $5.76 \pm 0.80$
\end{tabular}

${ }^{*}$ - determined on the basis of signals integration in temperatures: $25 / 35^{\circ} \mathrm{C}(298 / 308$ $[\mathrm{K}]) ; 35 / 45^{\circ} \mathrm{C}(308 / 318[\mathrm{~K}]) ; 45 / 55^{\circ} \mathrm{C}(318 / 328[\mathrm{~K}])$; for phenol signals calculations could not be carried out due to overlapping signals

** - determined on the basis of signals integration in temperatures: $-20 /-10{ }^{\circ} \mathrm{C}(253 / 263$ $[\mathrm{K}]) ;-10 / 0{ }^{\circ} \mathrm{C}(263 / 273[\mathrm{~K}]) ; 0 / 25^{\circ} \mathrm{C}(273 / 298[\mathrm{~K}])$; for amine signals calculations could not be carried out due to overlapping signals ${ }^{* * *}$ - determined on the basis of signals integration in temperatures: $5 / 15^{\circ} \mathrm{C}(278 / 288$ $[\mathrm{K}]) ; 15 / 25^{\circ} \mathrm{C}(288 / 298[\mathrm{~K}]) ; 25 / 35^{\circ} \mathrm{C}(298 / 308$ [K]) for amine signals calculations could not be carried out due to overlapping signals

$$
\Delta \mathrm{H}=\frac{-\mathrm{R}\left(\ln \mathrm{K}_{2}-\ln \mathrm{K}_{1}\right)}{\left(\frac{1}{\mathrm{~T}_{2}}-\frac{1}{\mathrm{~T}_{1}}\right)}
$$

$\mathrm{T}_{2}$ and $\mathrm{T}_{1}$ - temperature of NMR measurement in $\mathrm{K}$.

$\mathrm{K}_{2}$ and $\mathrm{K}_{1}$ - ratio of two forms of RFB conformers in $T_{2}$ and $T_{1}$, respectively, determined on the basis of signals' integrations in the ${ }^{1} \mathrm{H}$ NMR spectra. 
5. NMR spectra

\subsection{NMR spectra of RMP}

${ }^{1} \mathrm{H}$ and ${ }^{13} \mathrm{C}$ NMR spectra of RMP in $\mathrm{CDCl}_{3}$ in $25^{\circ} \mathrm{C}$
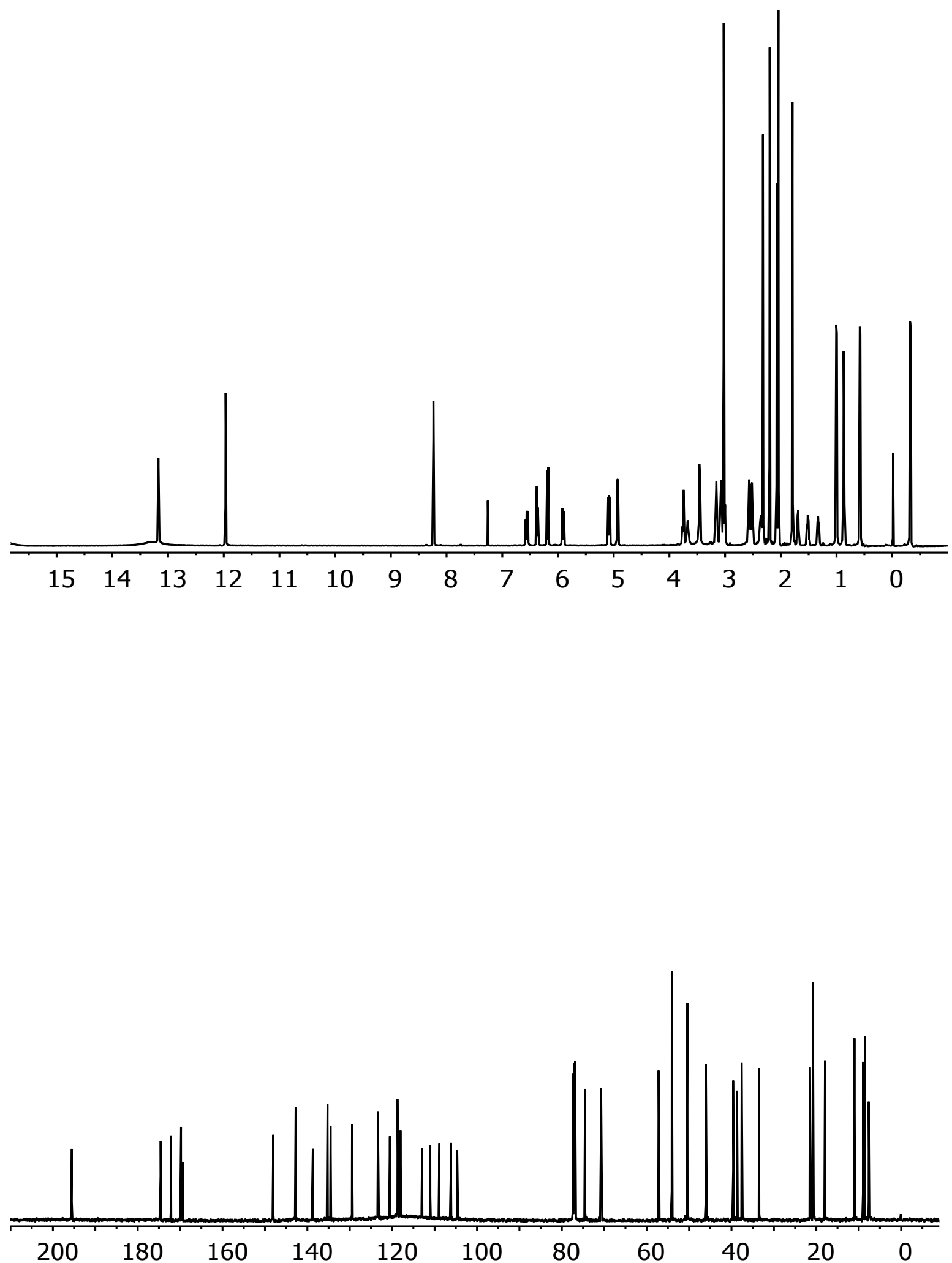
${ }^{1} \mathrm{H}$ and ${ }^{13} \mathrm{C}$ NMR spectra of RMP in DMSO- $\mathrm{d}_{6}+\mathrm{H}_{2} \mathrm{O}$ in $25^{\circ} \mathrm{C}$
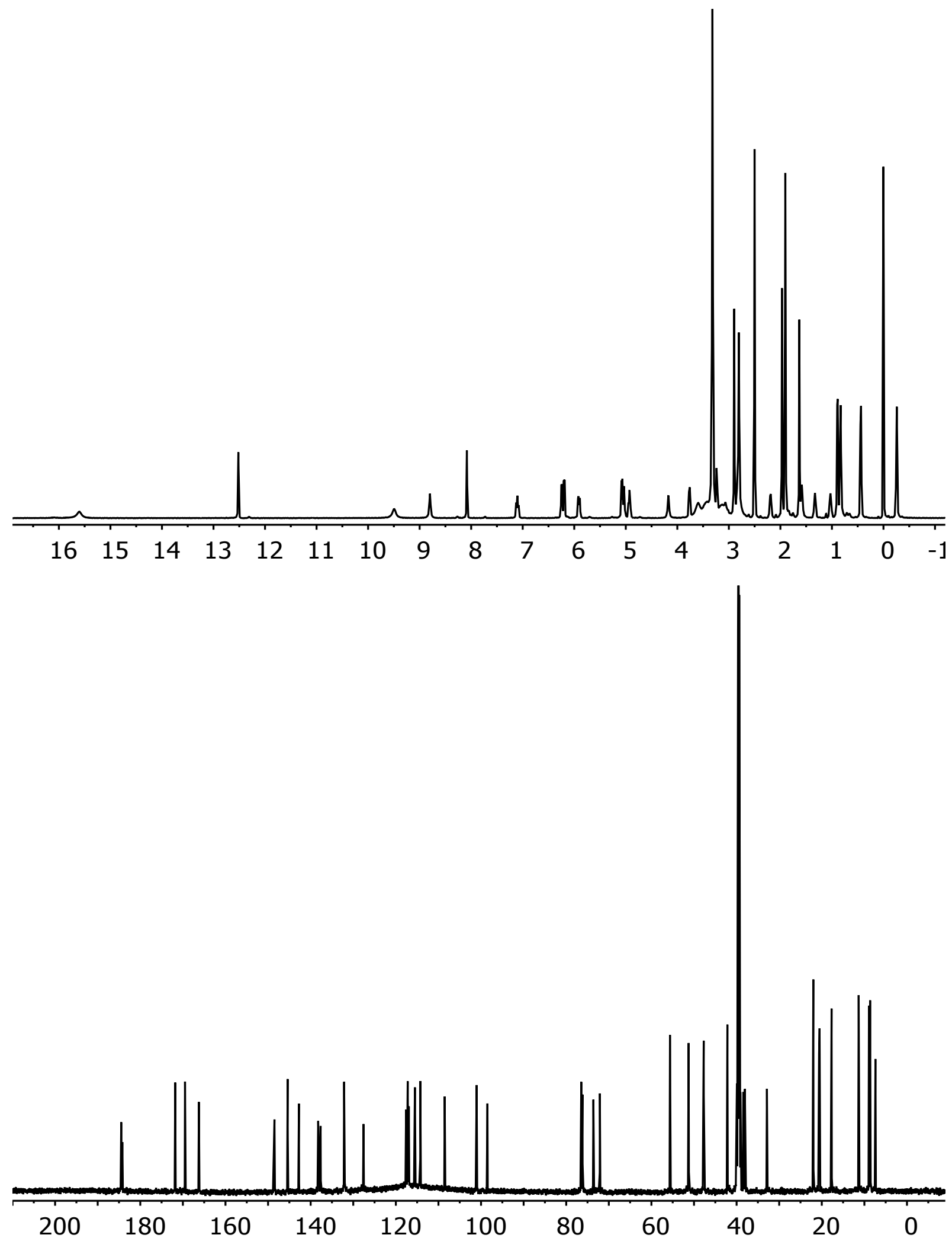


\subsection{NMR spectra of RFP}

${ }^{1} \mathrm{H}$ and ${ }^{13} \mathrm{C}$ NMR spectra of RFP in $\mathrm{CDCl}_{3}$ in $25^{\circ} \mathrm{C}$
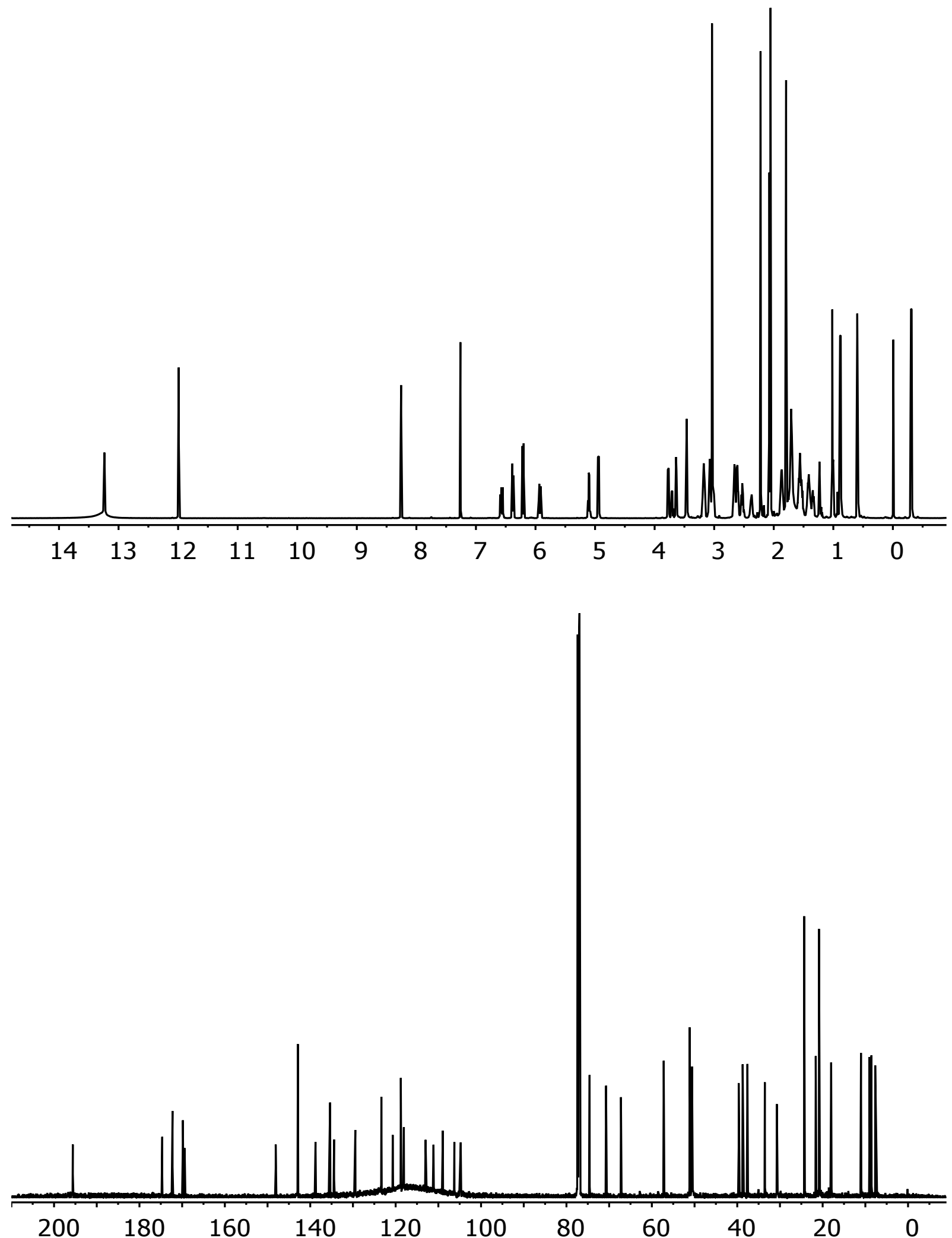
${ }^{1} \mathrm{H}$ and ${ }^{13} \mathrm{C}$ NMR spectra of RFP in DMSO- $\mathrm{d}_{6}+\mathrm{H}_{2} \mathrm{O}$ in $25^{\circ} \mathrm{C}$
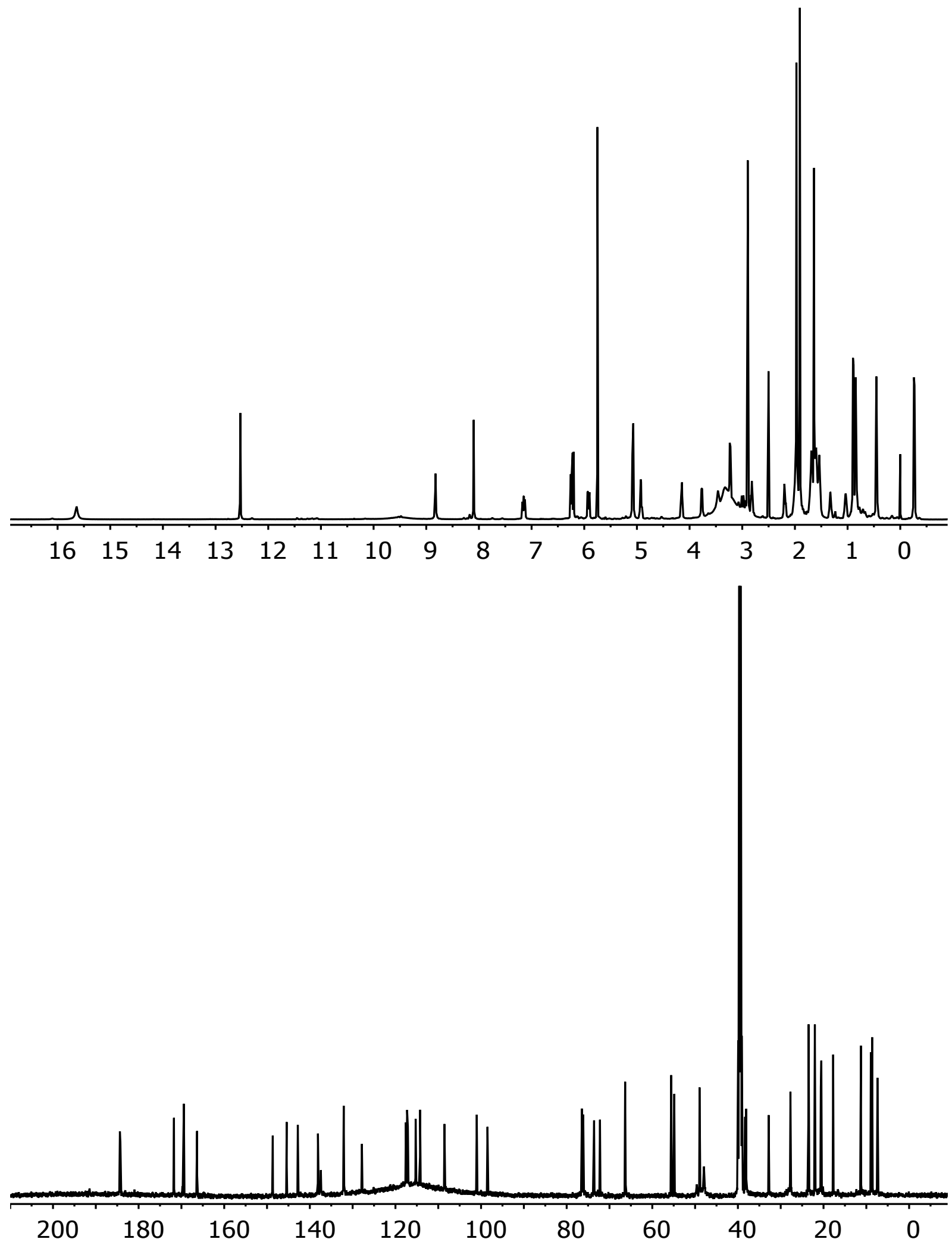


\subsection{NMR spectra of RFX}

${ }^{1} \mathrm{H}$ and ${ }^{13} \mathrm{C}$ NMR spectra of $\mathbf{R F X}$ in $\mathrm{CDCl}_{3}$ in $25^{\circ} \mathrm{C}$
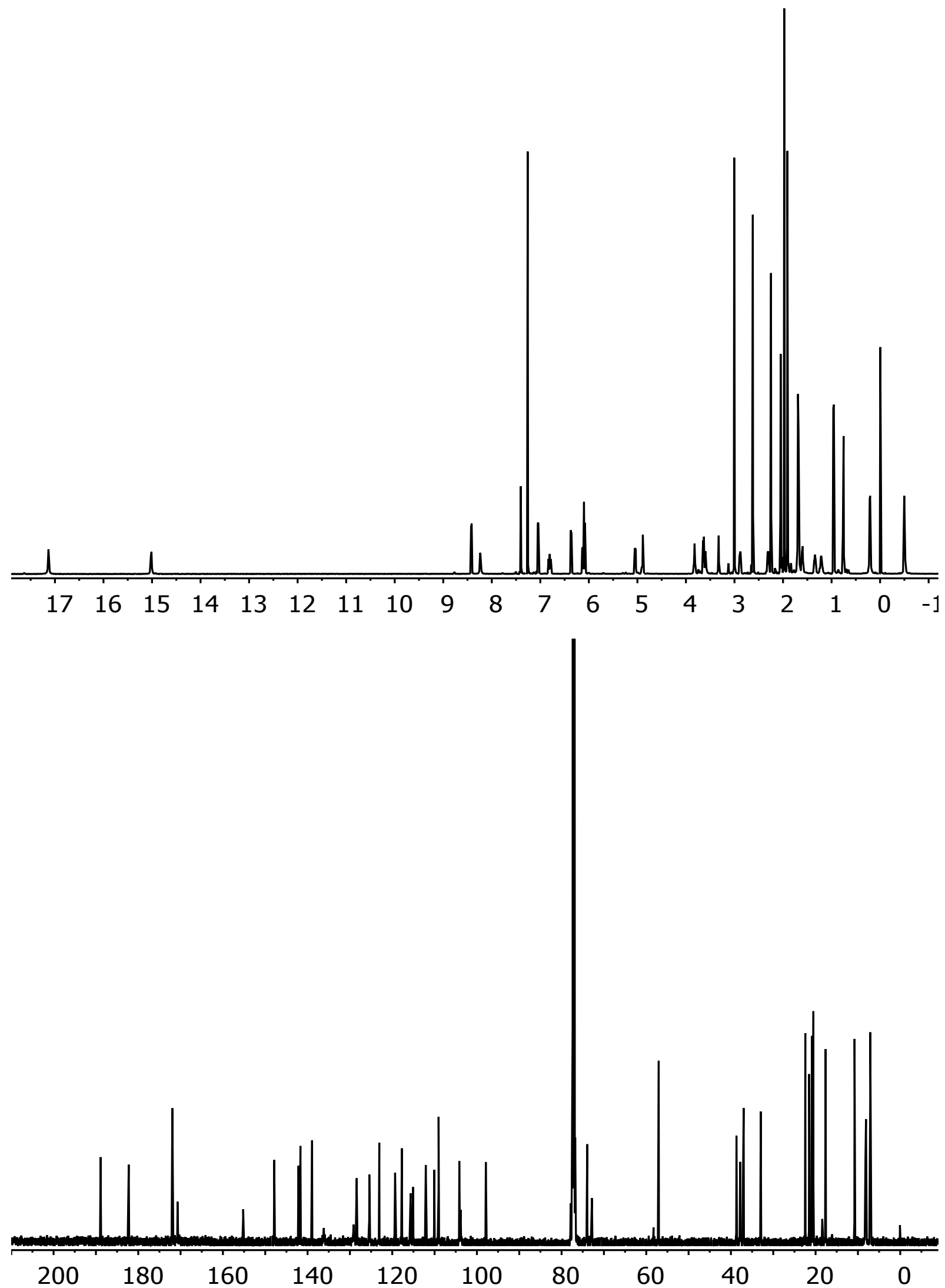
${ }^{1} \mathrm{H}$ and ${ }^{13} \mathrm{C}$ NMR spectrum of $\mathbf{R F X}$ in $\mathrm{CDCl}_{3}$ in $-25^{\circ} \mathrm{C}$

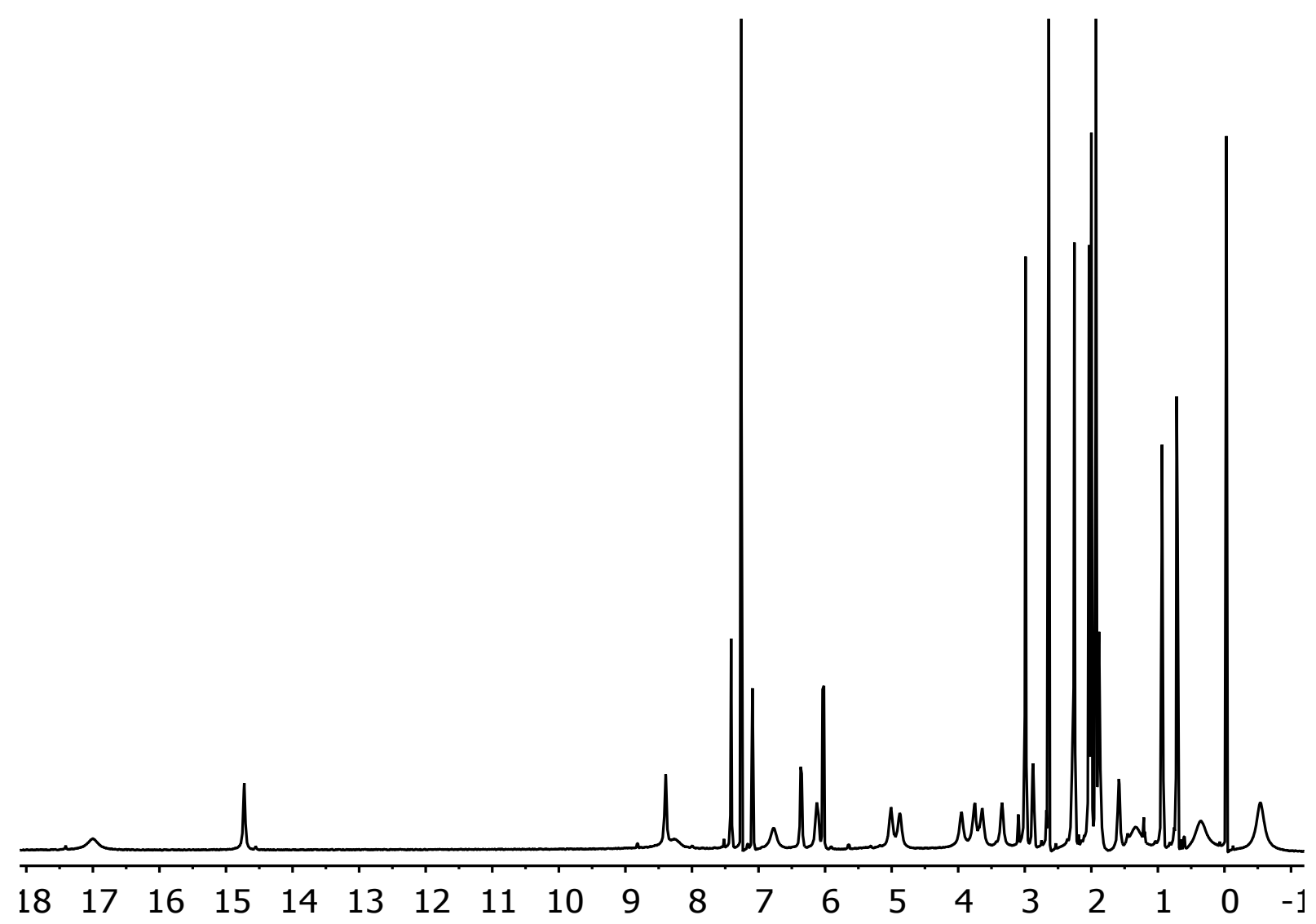


${ }^{1} \mathrm{H}$ and ${ }^{13} \mathrm{C}$ NMR spectra of RFX in DMSO- $\mathrm{d}_{6}+\mathrm{H}_{2} \mathrm{O}$ in $25^{\circ} \mathrm{C}$
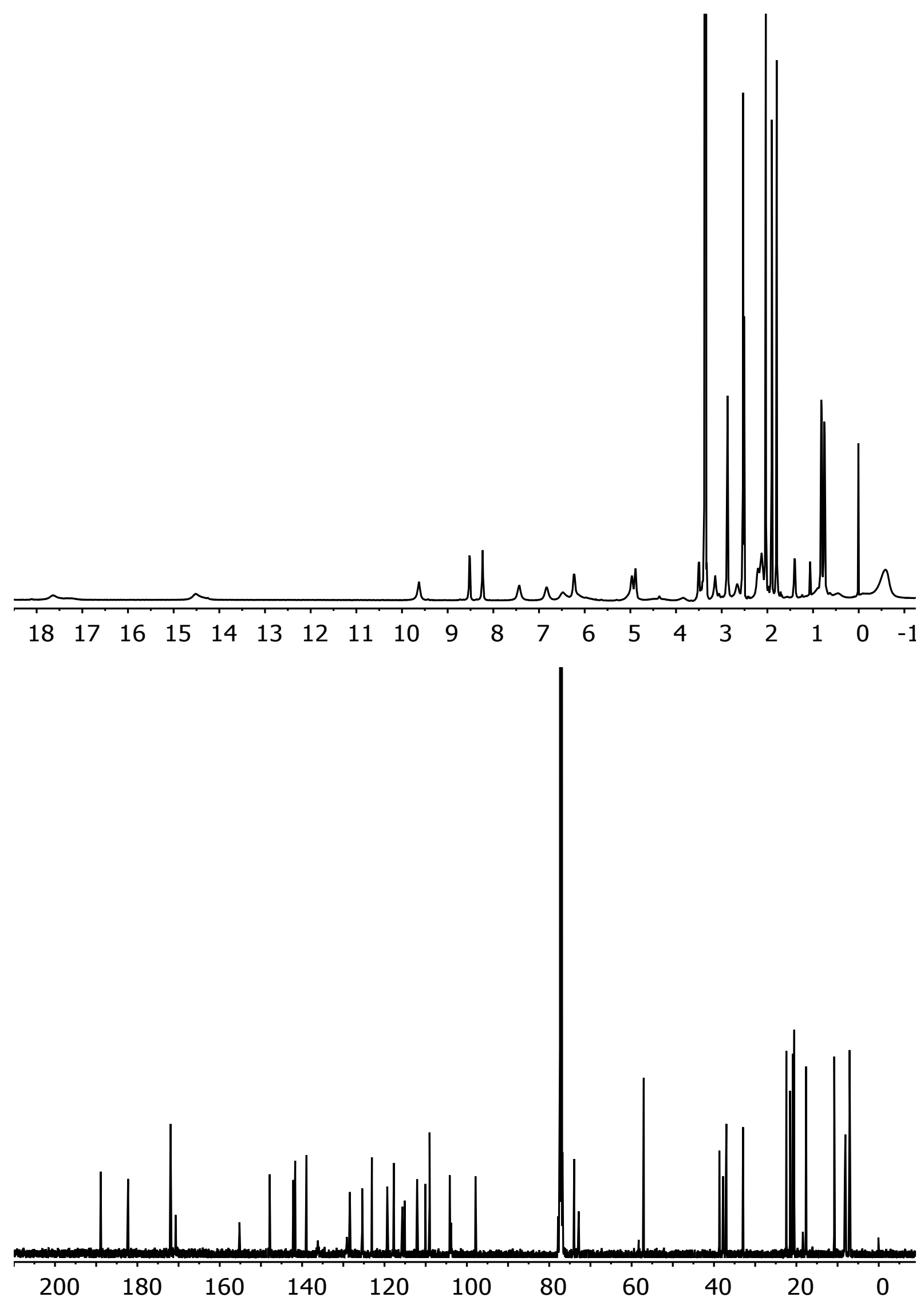
${ }^{1} \mathrm{H}$ and ${ }^{13} \mathrm{C}$ NMR spectra of RFX in $\mathrm{CD}_{3} \mathrm{CN}$ :DMSO- $\mathrm{d}_{6}+\mathrm{H}_{2} \mathrm{O}$ in $-30^{\circ} \mathrm{C}$
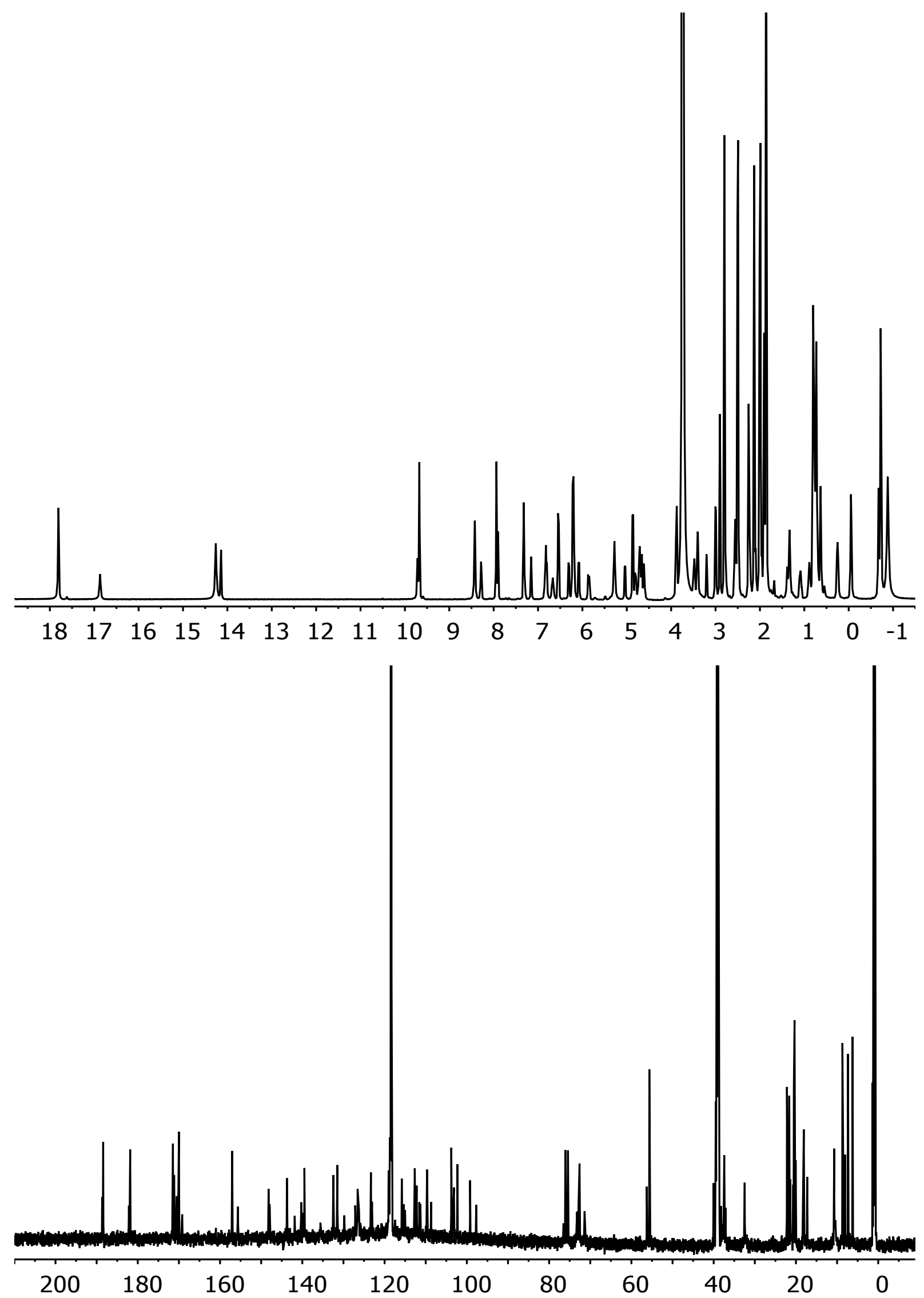
${ }^{1} \mathrm{H}$ spectrum of $\mathbf{R F X}$ in $\mathrm{CD}_{3} \mathrm{CN}+\mathrm{H}_{2} \mathrm{O}$ in $25^{\circ} \mathrm{C}$

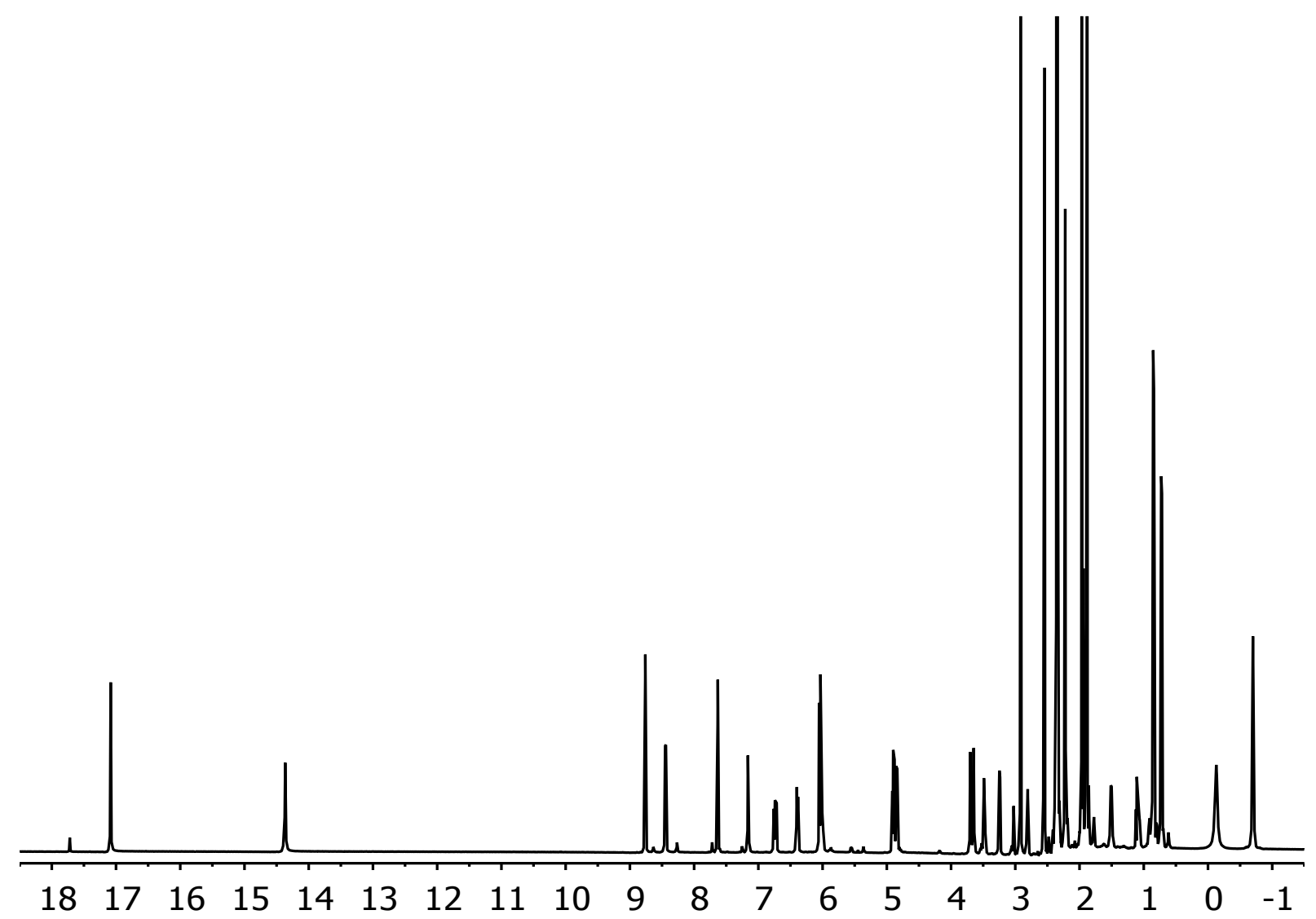


${ }^{1} \mathrm{H}$ and ${ }^{13} \mathrm{C}$ NMR spectrum of RFX in $\mathrm{CD}_{3} \mathrm{CN}+\mathrm{H}_{2} \mathrm{O}$ in $15^{\circ} \mathrm{C}$

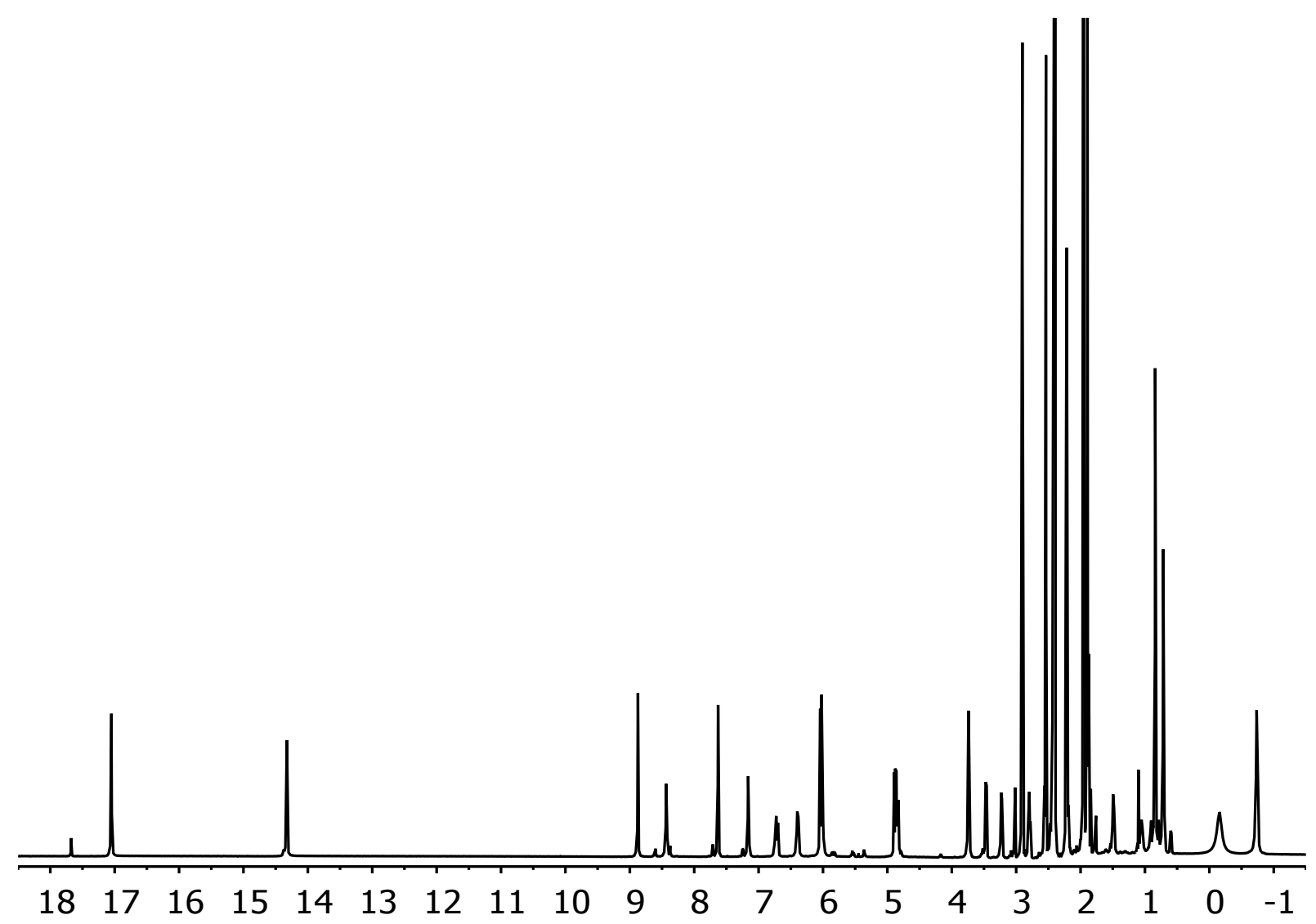


${ }^{1} \mathrm{H}$ and ${ }^{13} \mathrm{C}$ NMR spectrum of $\mathbf{R F X}$ in $\mathrm{CD}_{3} \mathrm{CN}+\mathrm{H}_{2} \mathrm{O}$ in $5^{\circ} \mathrm{C}$

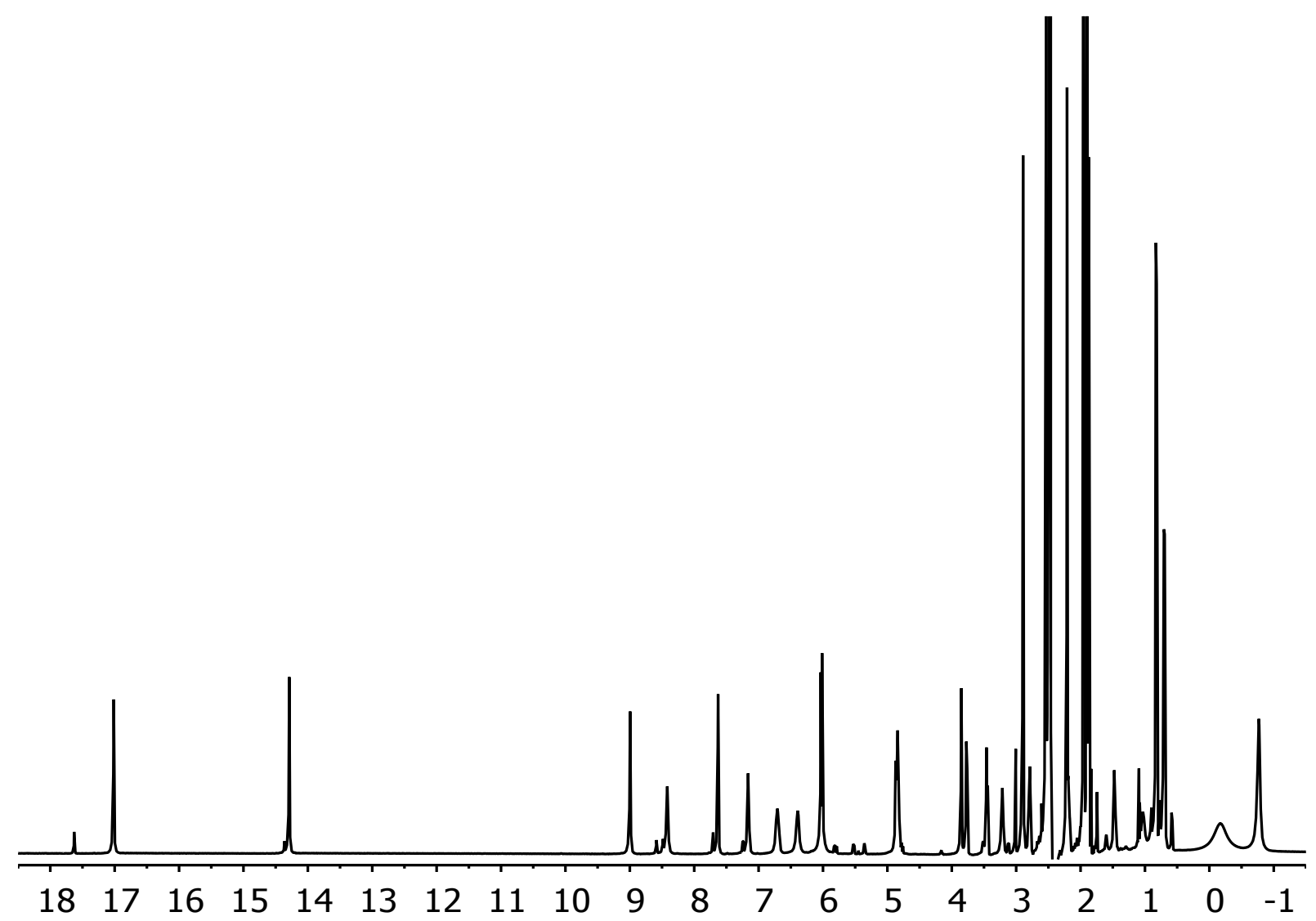


${ }^{1} \mathrm{H}$ and ${ }^{13} \mathrm{C}$ NMR spectrum of RFX in $\mathrm{CD}_{3} \mathrm{CN}+\mathrm{H}_{2} \mathrm{O}$ in $0^{\circ} \mathrm{C}$

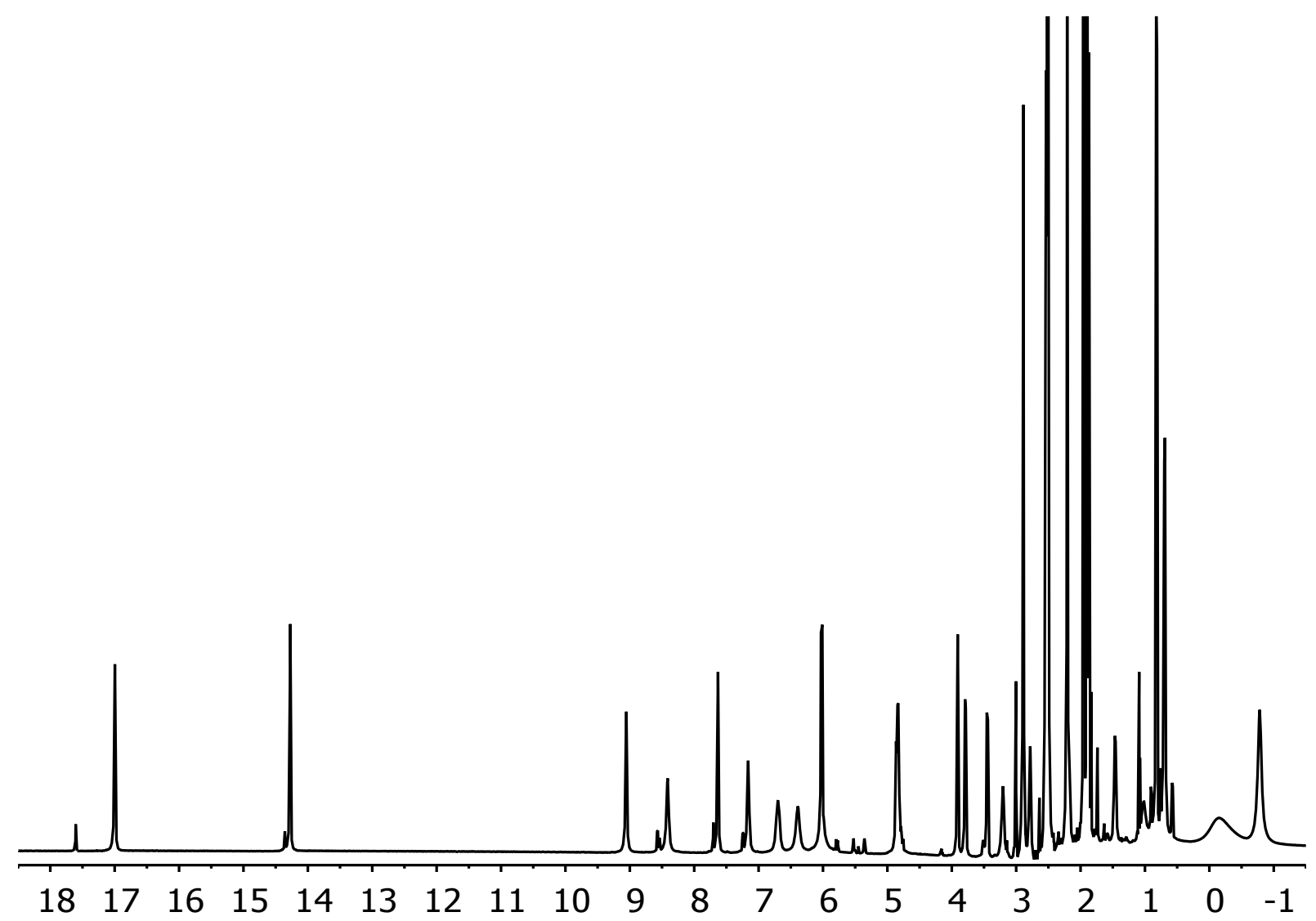


S45

${ }^{1} \mathrm{H}$ and ${ }^{13} \mathrm{C}$ NMR spectrum of RFX in $\mathrm{CD}_{3} \mathrm{CN}+\mathrm{H}_{2} \mathrm{O}$ in $-10^{\circ} \mathrm{C}$

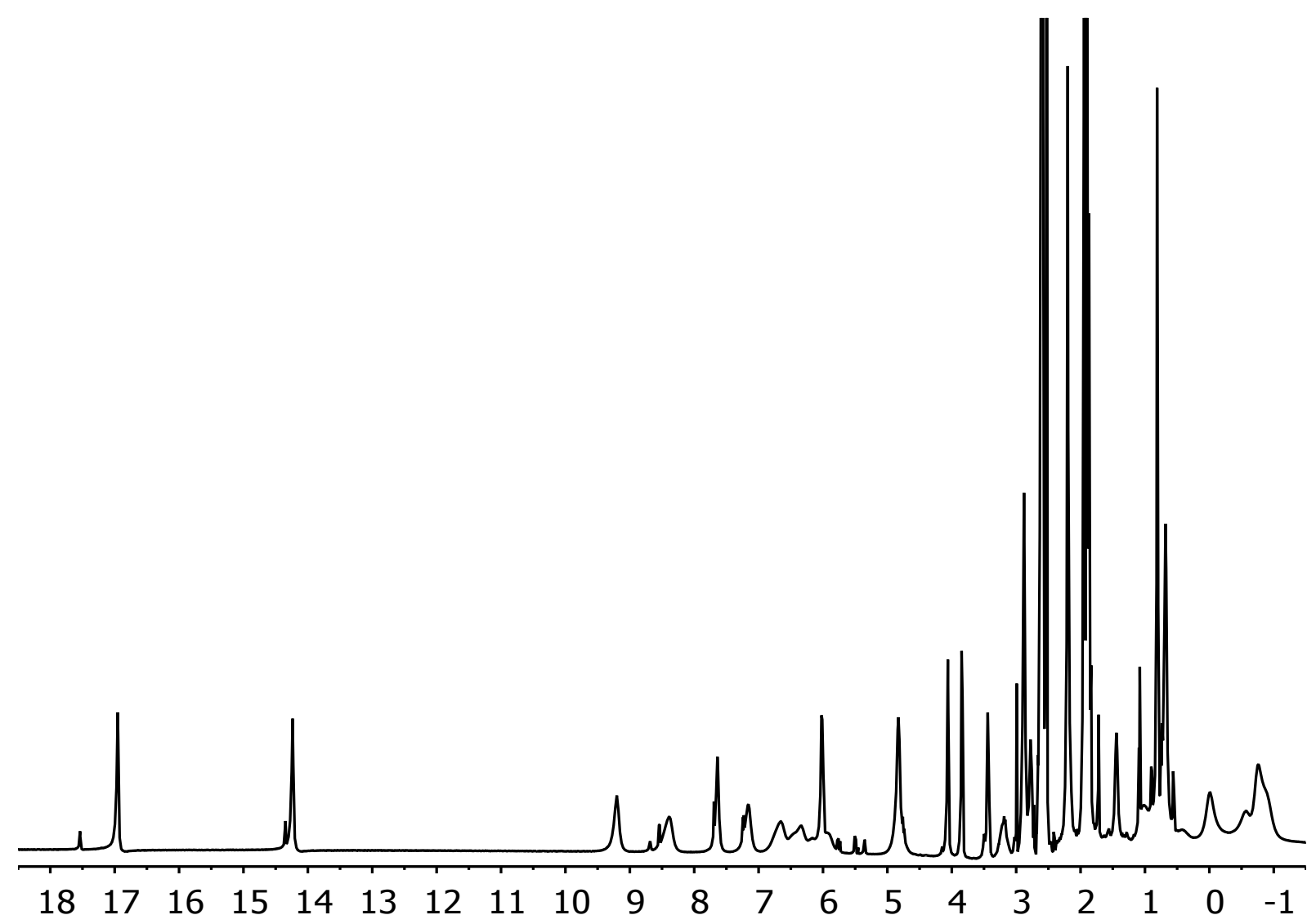


${ }^{1} \mathrm{H}$ and ${ }^{13} \mathrm{C}$ NMR spectrum of RFX in $\mathrm{CD}_{3} \mathrm{CN}+\mathrm{H}_{2} \mathrm{O}$ in $-20^{\circ} \mathrm{C}$

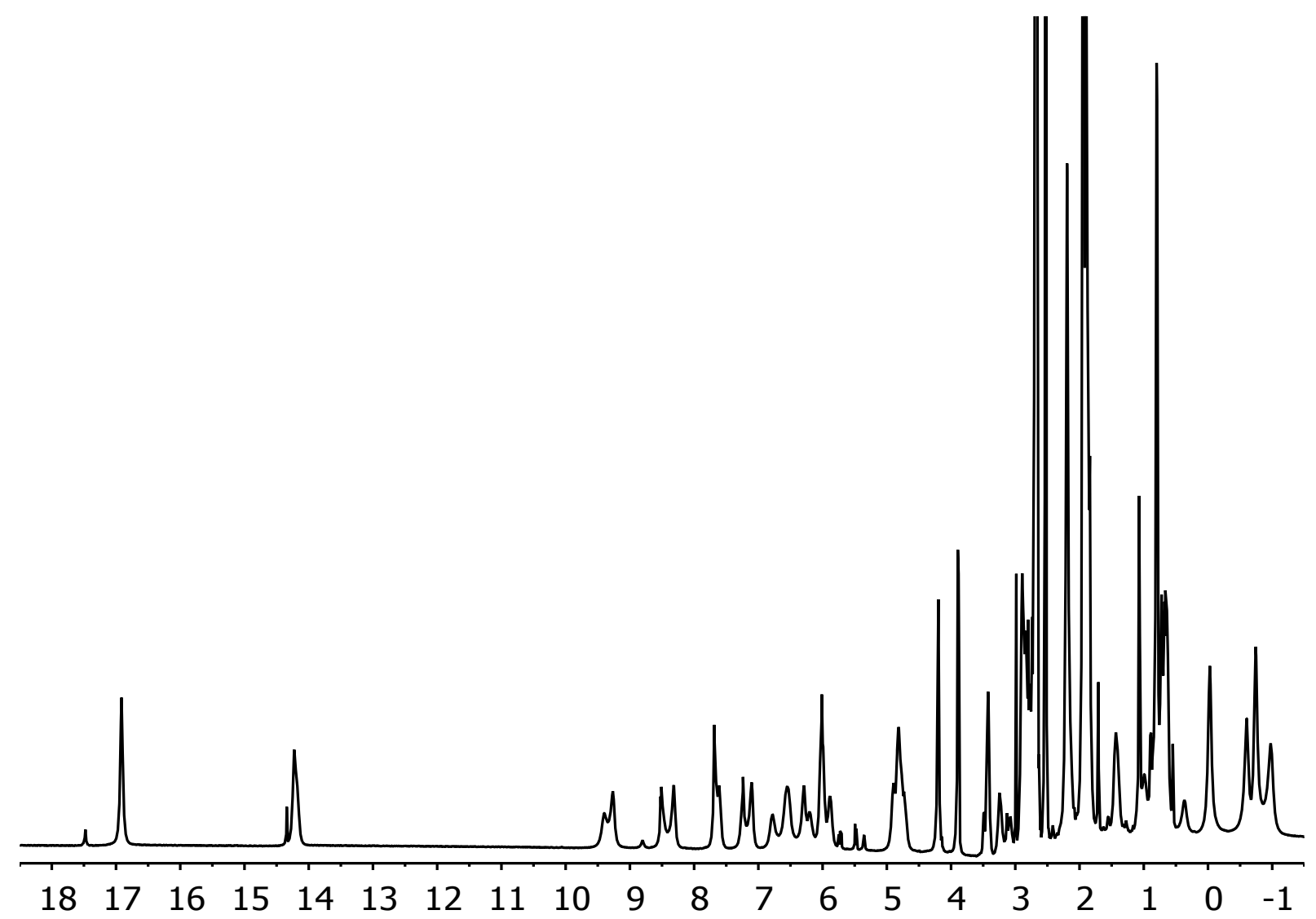


${ }^{1} \mathrm{H}$ and ${ }^{13} \mathrm{C}$ NMR spectrum of RFX in $\mathrm{CD}_{3} \mathrm{CN}+\mathrm{H}_{2} \mathrm{O}$ in $-30^{\circ} \mathrm{C}$

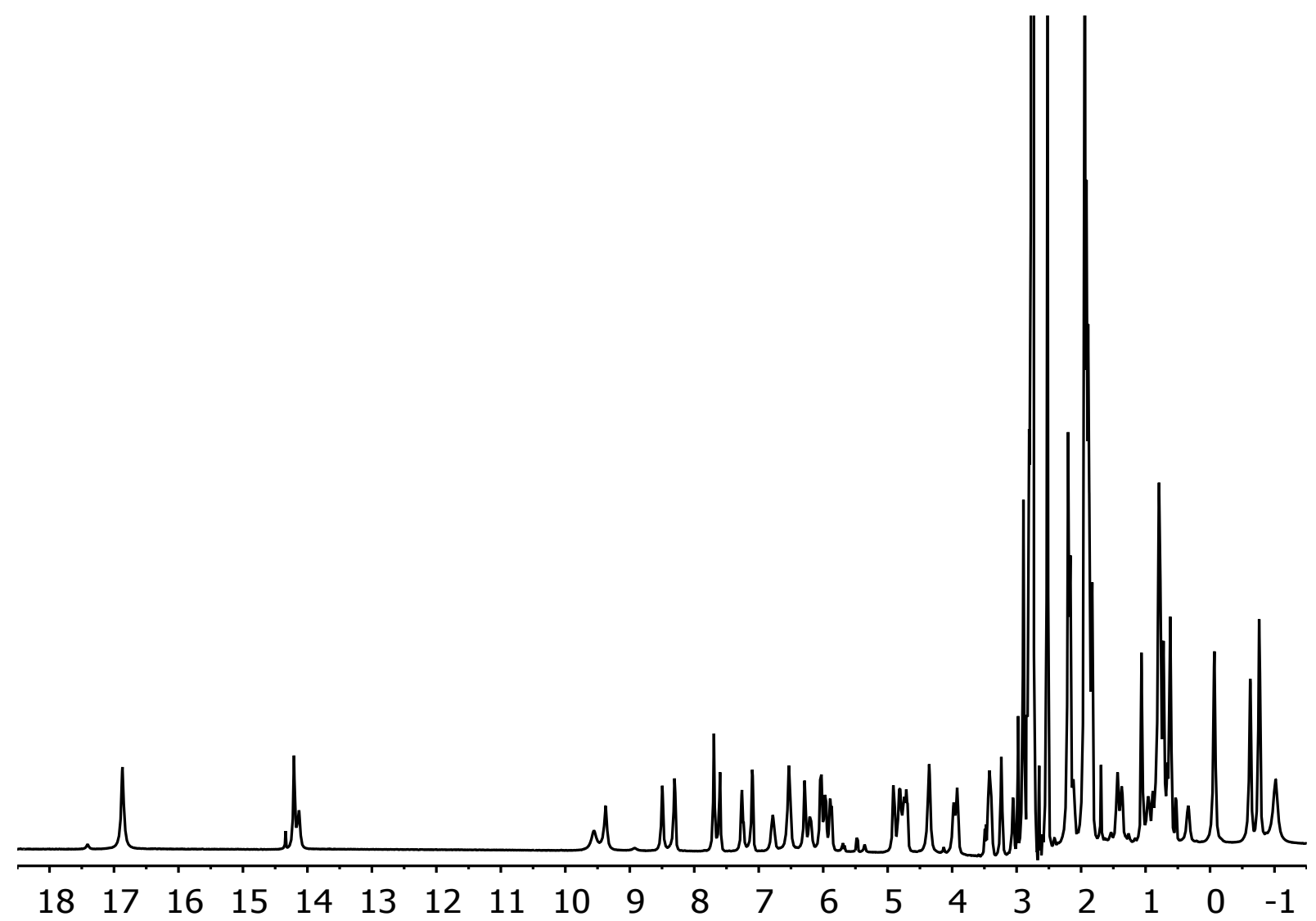




\subsection{NMR spectra of RFB}

${ }^{1} \mathrm{H}$ and ${ }^{13} \mathrm{C}$ NMR spectra of RFB in $\mathrm{CDCl}_{3}$ in $25^{\circ} \mathrm{C}$
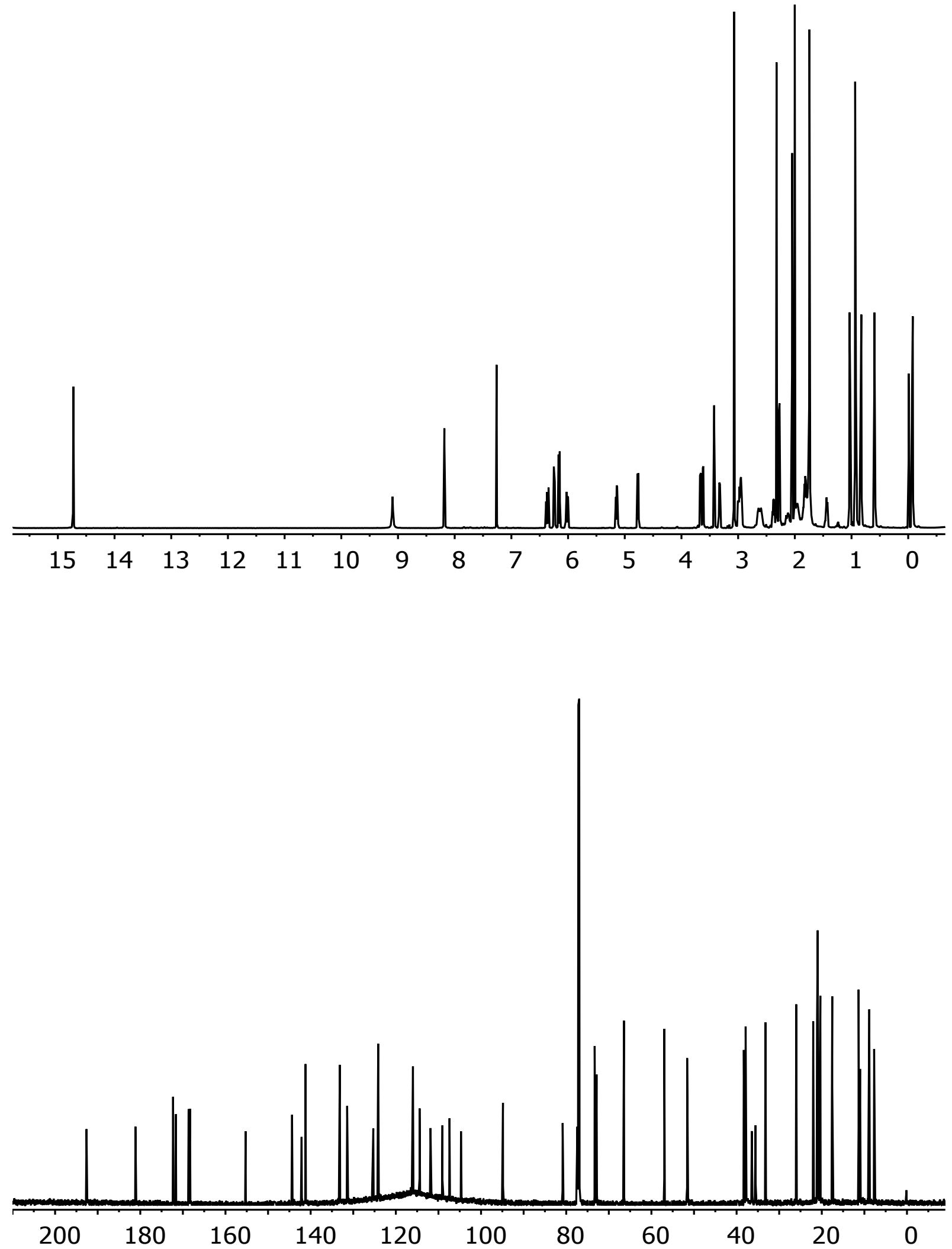
${ }^{1} \mathrm{H}$ and ${ }^{13} \mathrm{C}$ NMR spectra of RFB in DMSO- $\mathrm{d}_{6}+\mathrm{H}_{2} \mathrm{O}$ in $25^{\circ} \mathrm{C}$
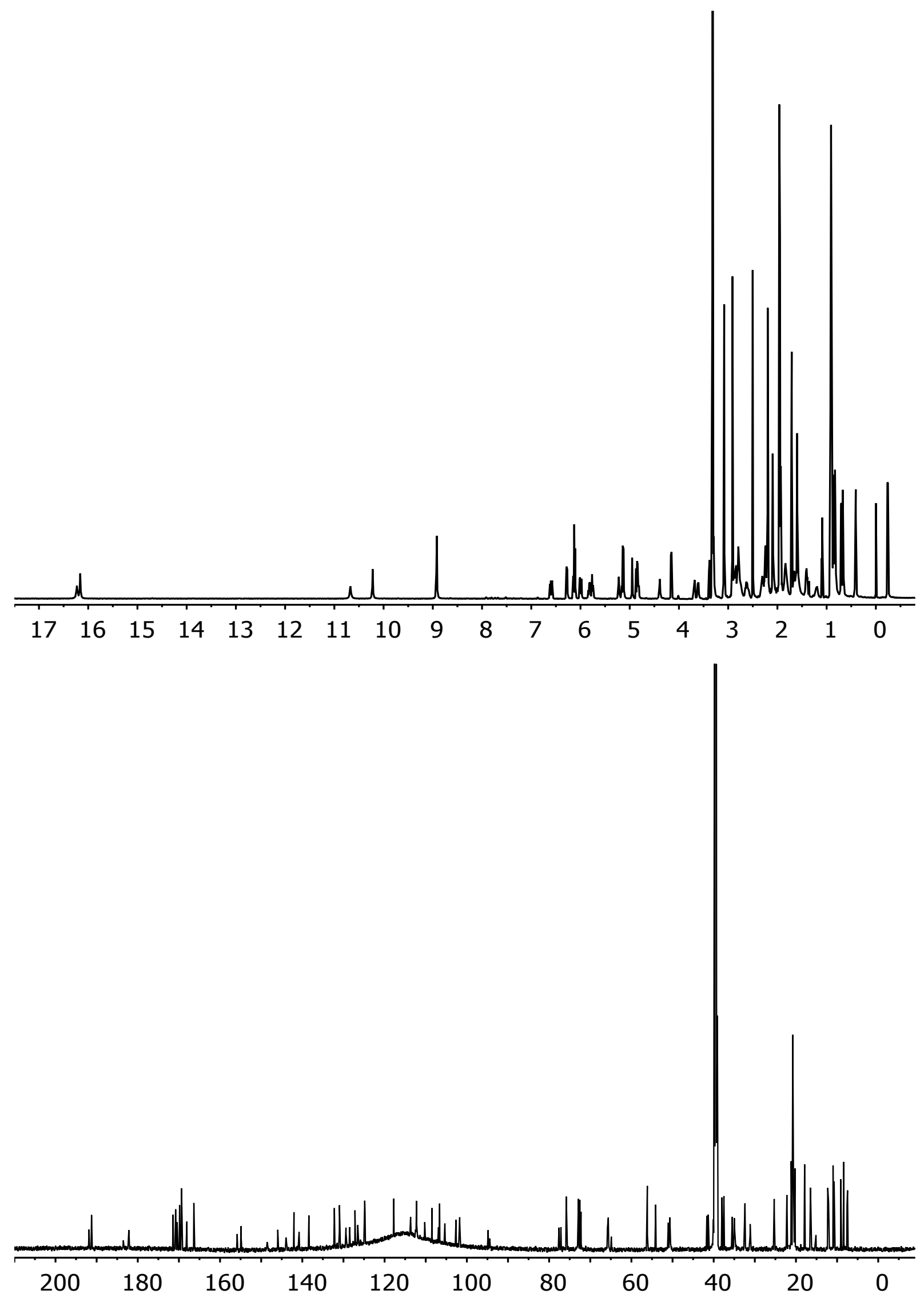
${ }^{1} \mathrm{H}$ spectrum of RFB in DMSO- $\mathrm{d}_{6}+\mathrm{H}_{2} \mathrm{O}$ in $25^{\circ} \mathrm{C}$

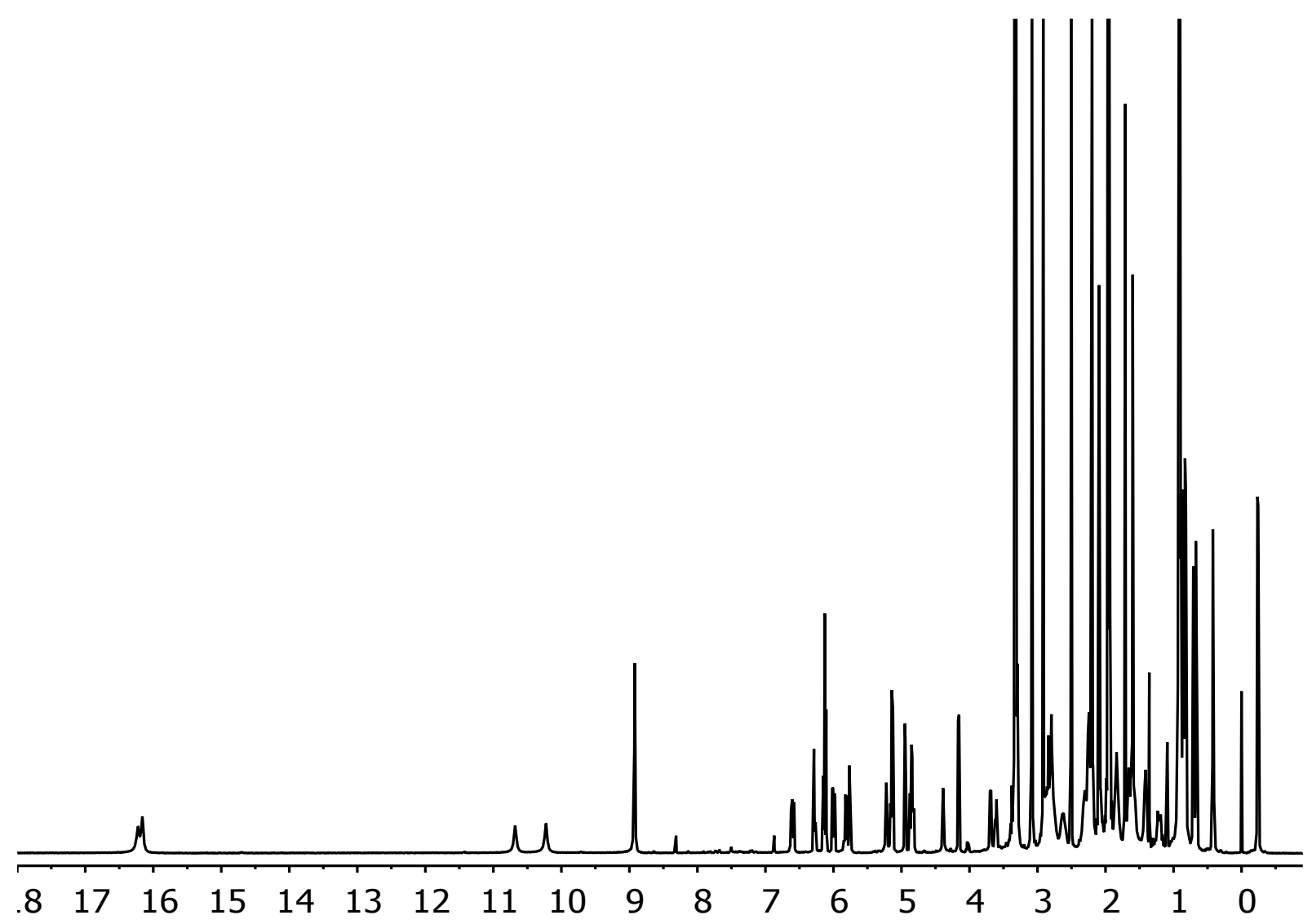


${ }^{1} \mathrm{H}$ spectrum of RFB in DMSO- $\mathrm{d}_{6}+\mathrm{H}_{2} \mathrm{O}$ in $35^{\circ} \mathrm{C}$

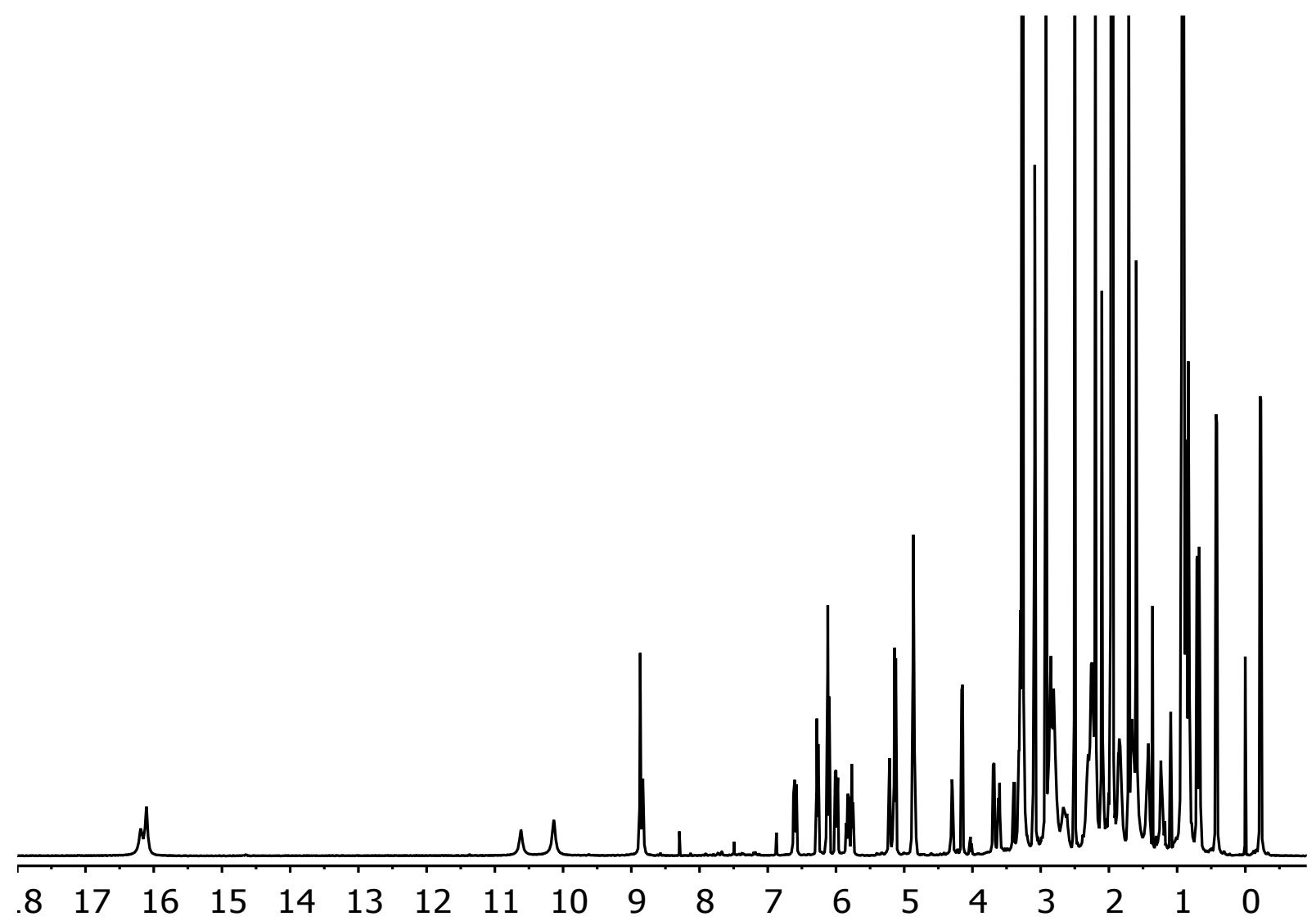


${ }^{1} \mathrm{H}$ spectrum of RFB in DMSO- $\mathrm{d}_{6}+\mathrm{H}_{2} \mathrm{O}$ in $45^{\circ} \mathrm{C}$

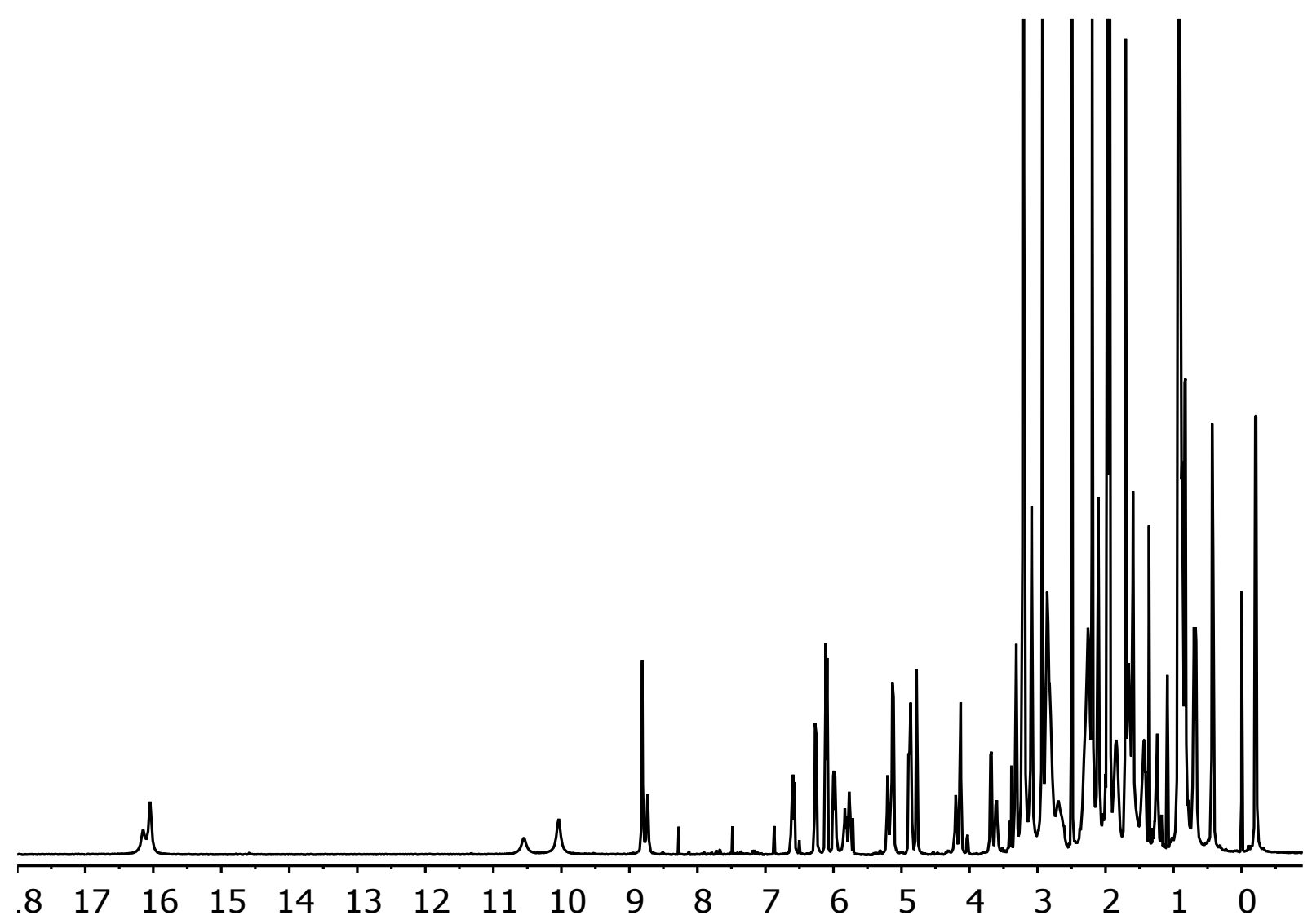


${ }^{1} \mathrm{H}$ spectrum of RFB in DMSO- $\mathrm{d}_{6}+\mathrm{H}_{2} \mathrm{O}$ in $55^{\circ} \mathrm{C}$

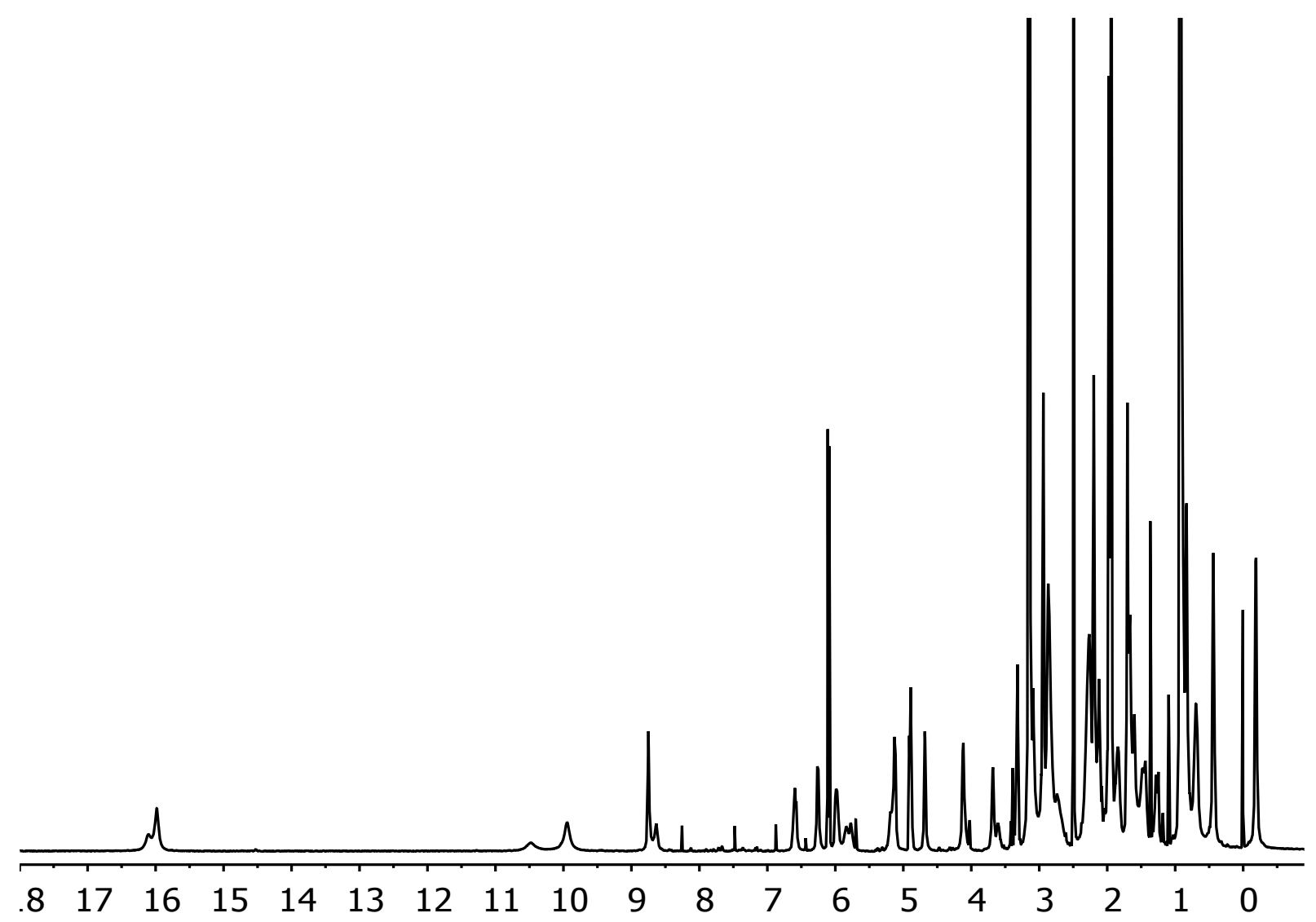


${ }^{1} \mathrm{H}$ spectrum of $\mathbf{R F B}$ in $\mathrm{CD}_{3} \mathrm{CN}+\mathrm{H}_{2} \mathrm{O}$ in $25^{\circ} \mathrm{C}$

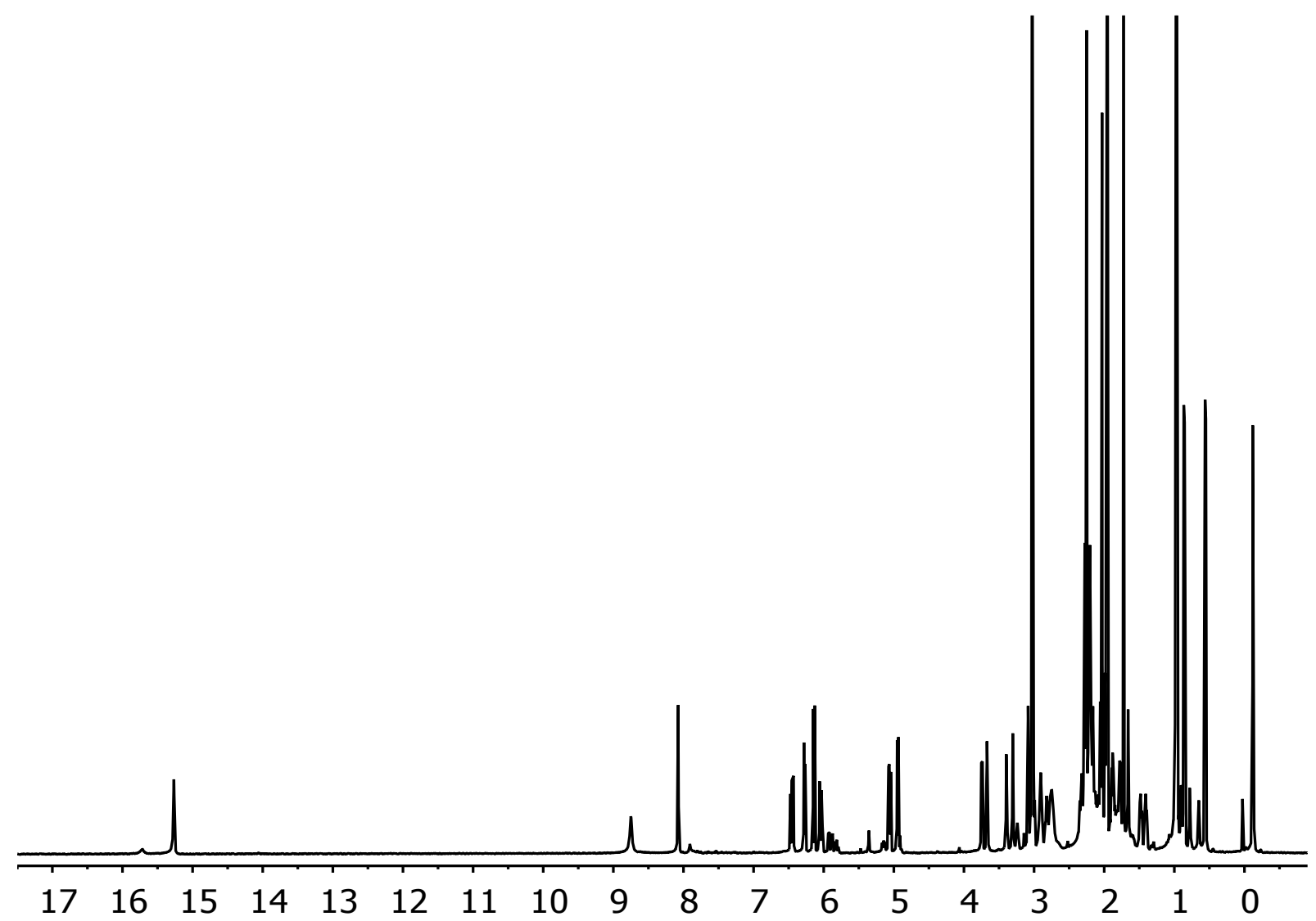


${ }^{1} \mathrm{H}$ spectrum of RFB in $\mathrm{CD}_{3} \mathrm{CN}+\mathrm{H}_{2} \mathrm{O}$ in $0^{\circ} \mathrm{C}$

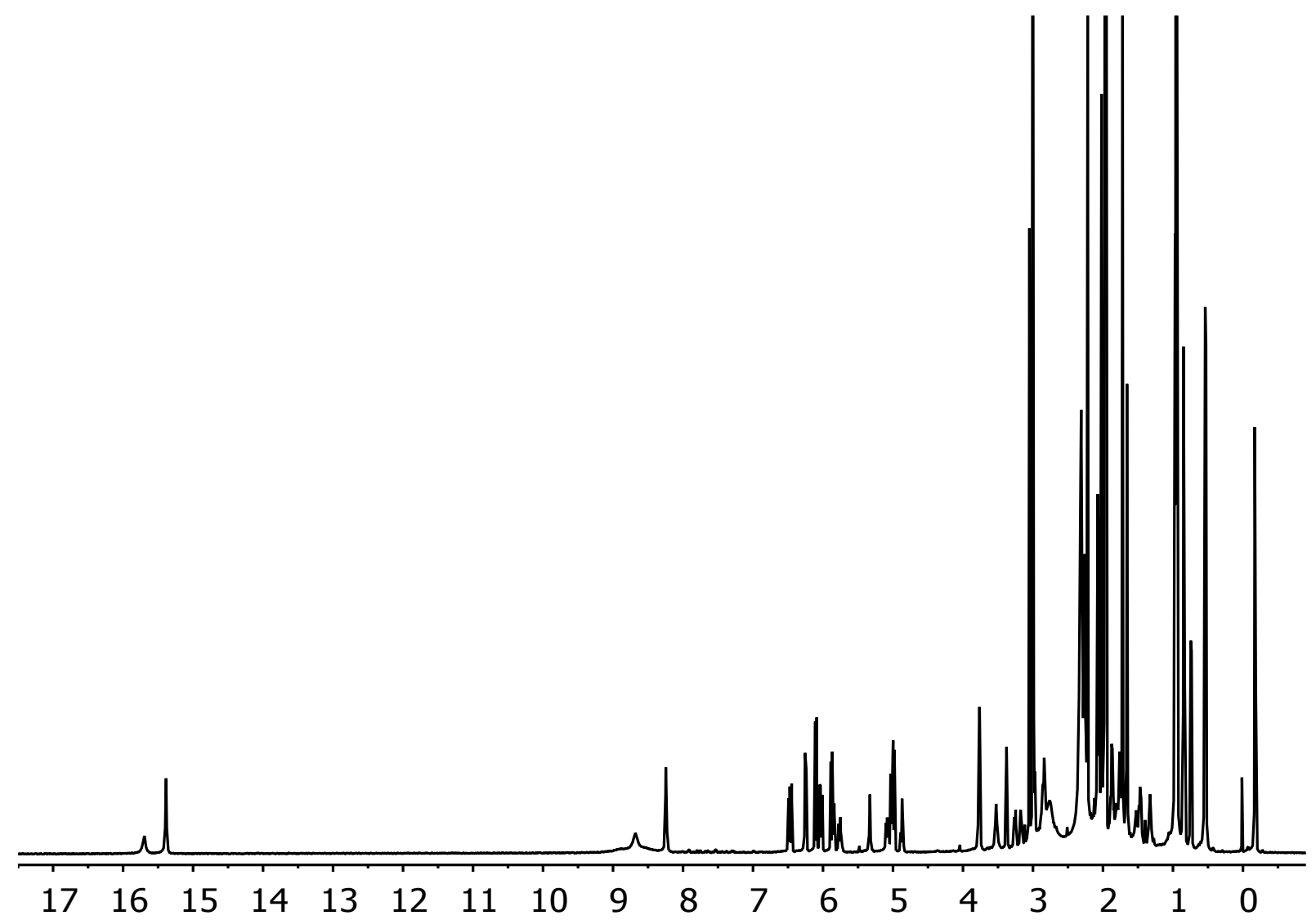


${ }^{1} \mathrm{H}$ spectrum of RFB in $\mathrm{CD}_{3} \mathrm{CN}+\mathrm{H}_{2} \mathrm{O}$ in $-10^{\circ} \mathrm{C}$

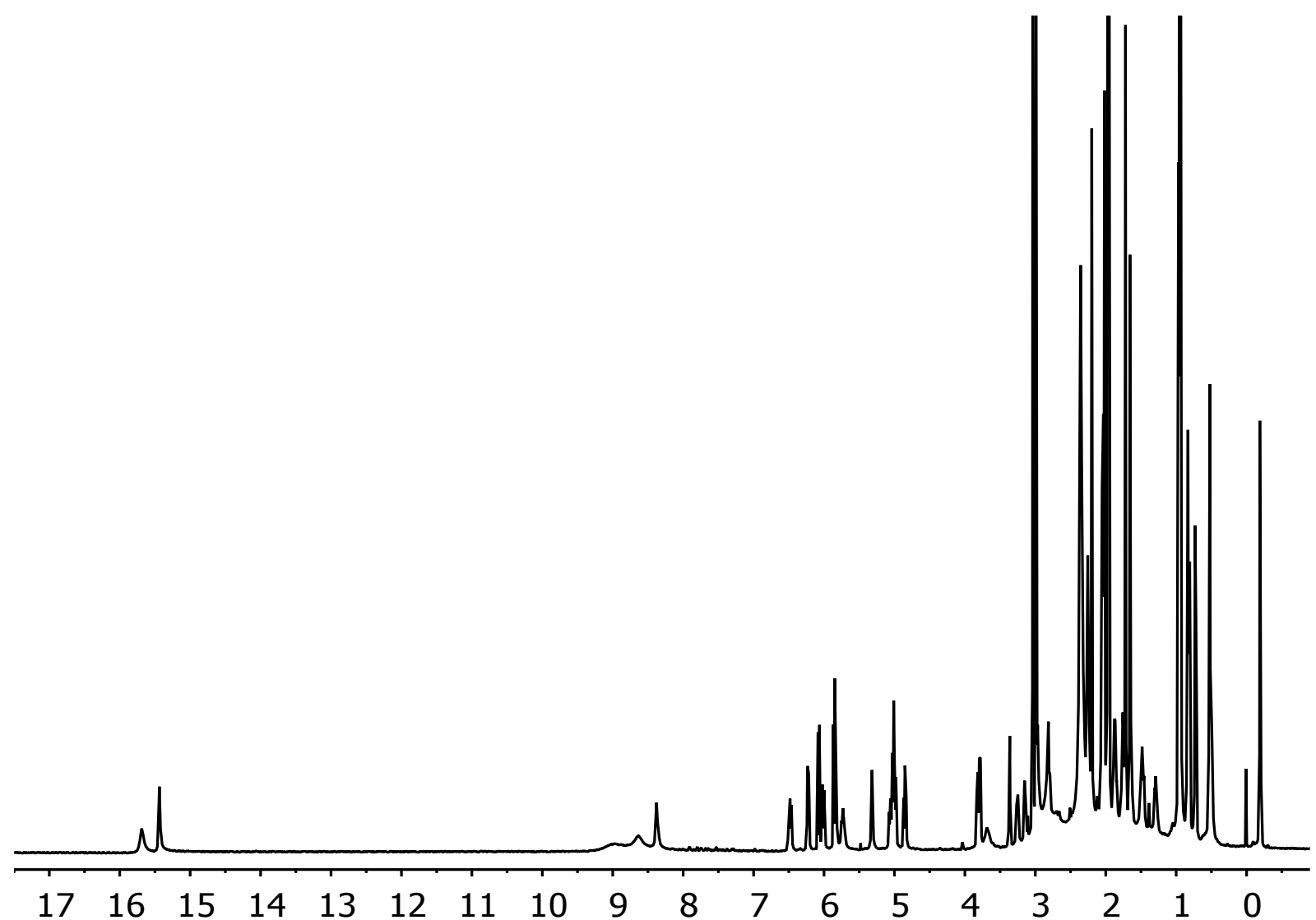


${ }^{1} \mathrm{H}$ spectrum of RFB in $\mathrm{CD}_{3} \mathrm{CN}+\mathrm{H}_{2} \mathrm{O}$ in $-20^{\circ} \mathrm{C}$

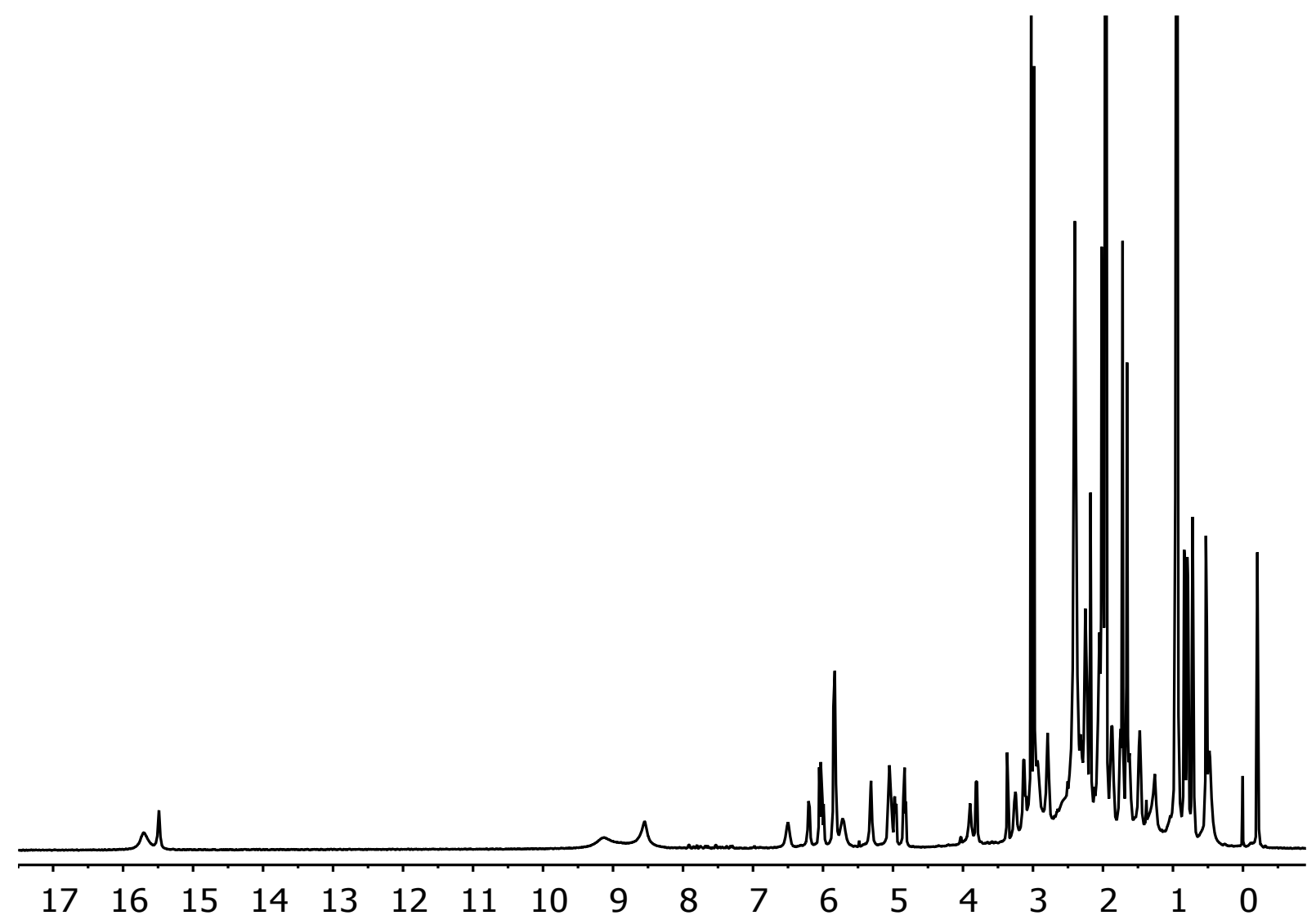


${ }^{1} \mathrm{H}$ NMR spectrum of RFB in $\mathrm{CD}_{3} \mathrm{CN}$ :DMSO- $\mathrm{d}_{6}+\mathrm{H}_{2} \mathrm{O}$ in $65^{\circ} \mathrm{C}$

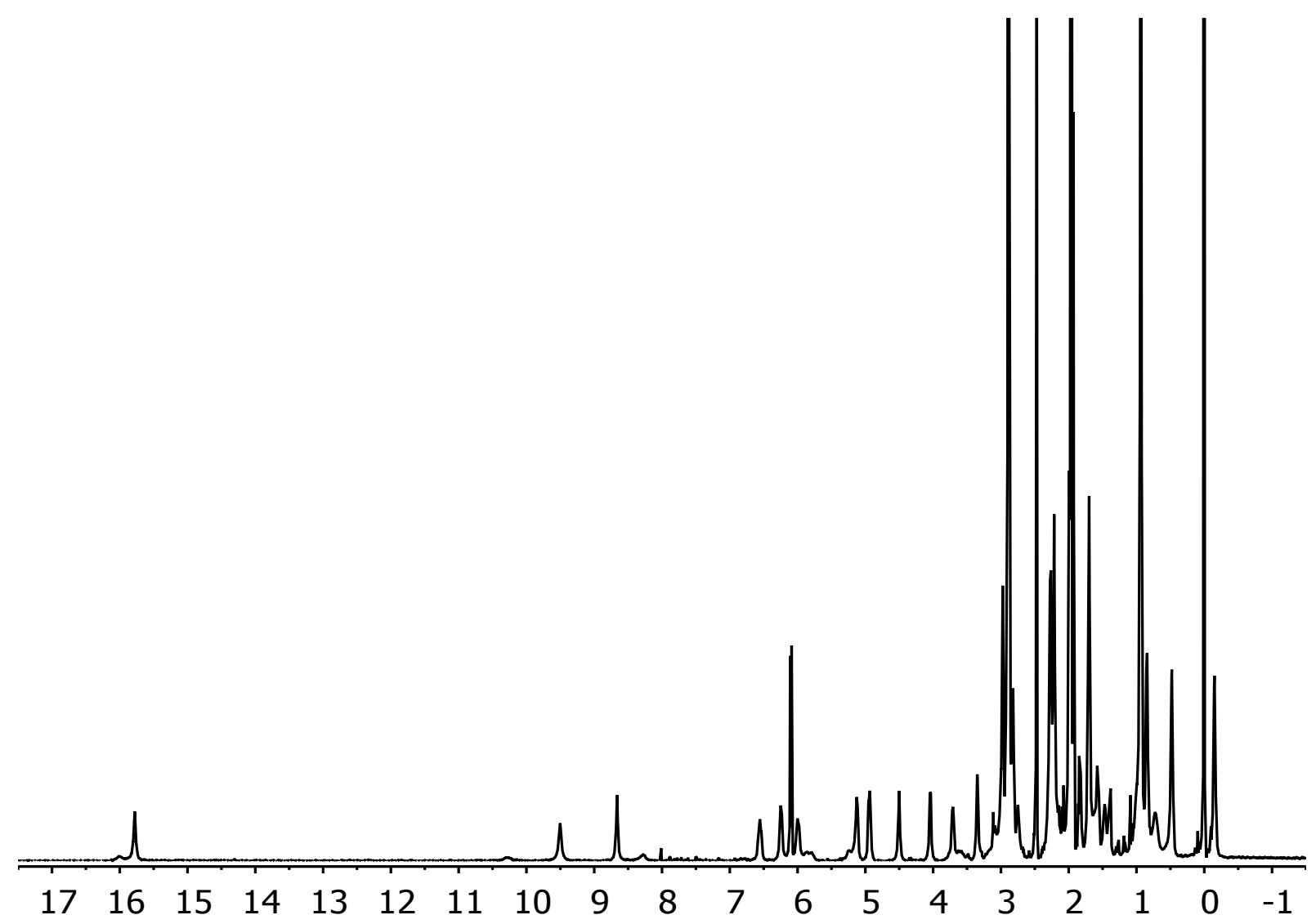


${ }^{1} \mathrm{H}$ NMR spectrum of RFB in $\mathrm{CD}_{3} \mathrm{CN}: D M S O-\mathrm{d}_{6}+\mathrm{H}_{2} \mathrm{O}$ in $55^{\circ} \mathrm{C}$

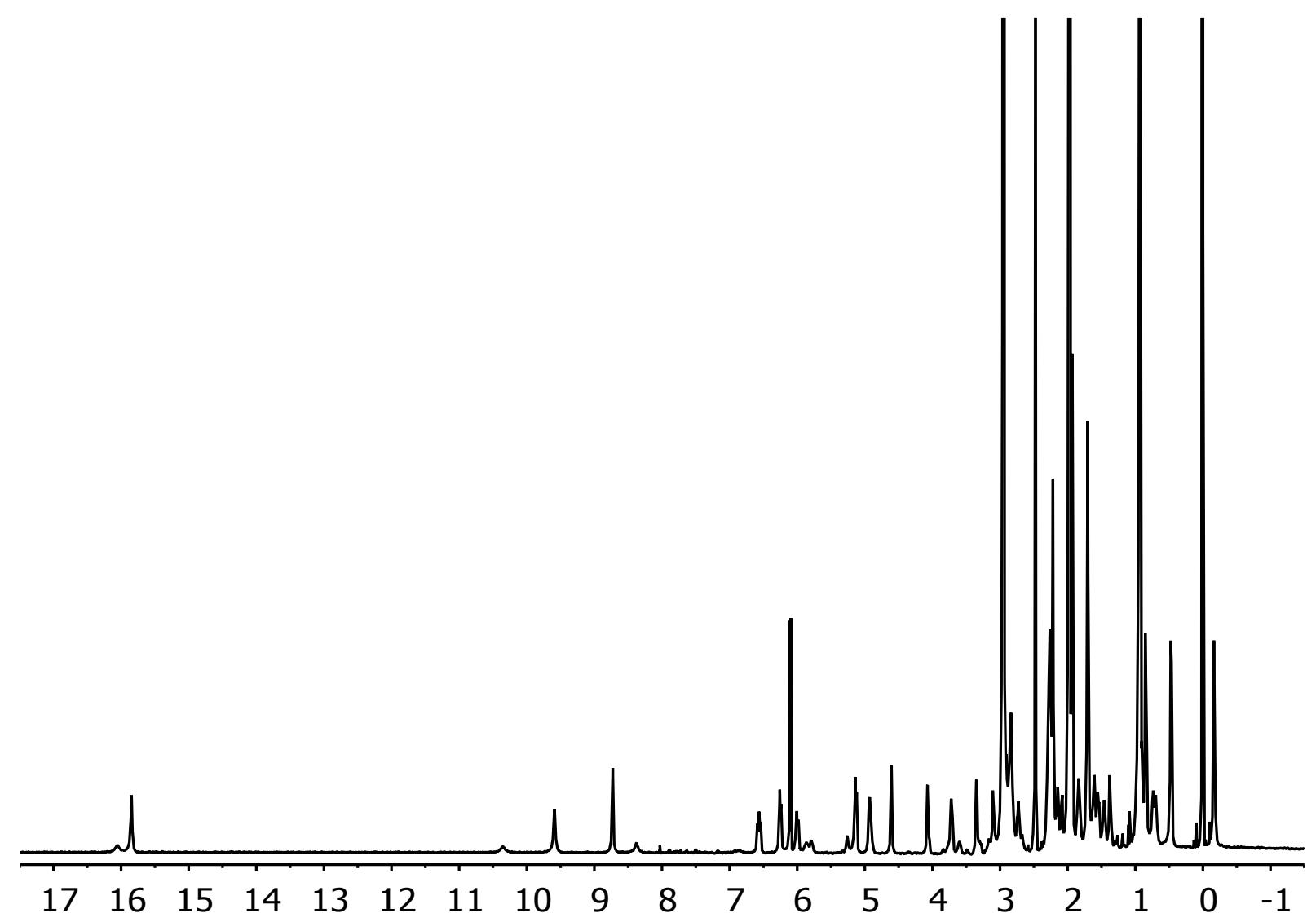


${ }^{1} \mathrm{H}$ NMR spectrum of RFB in $\mathrm{CD}_{3} \mathrm{CN}: \mathrm{DMSO}-\mathrm{d}_{6}+\mathrm{H}_{2} \mathrm{O}$ in $45^{\circ} \mathrm{C}$

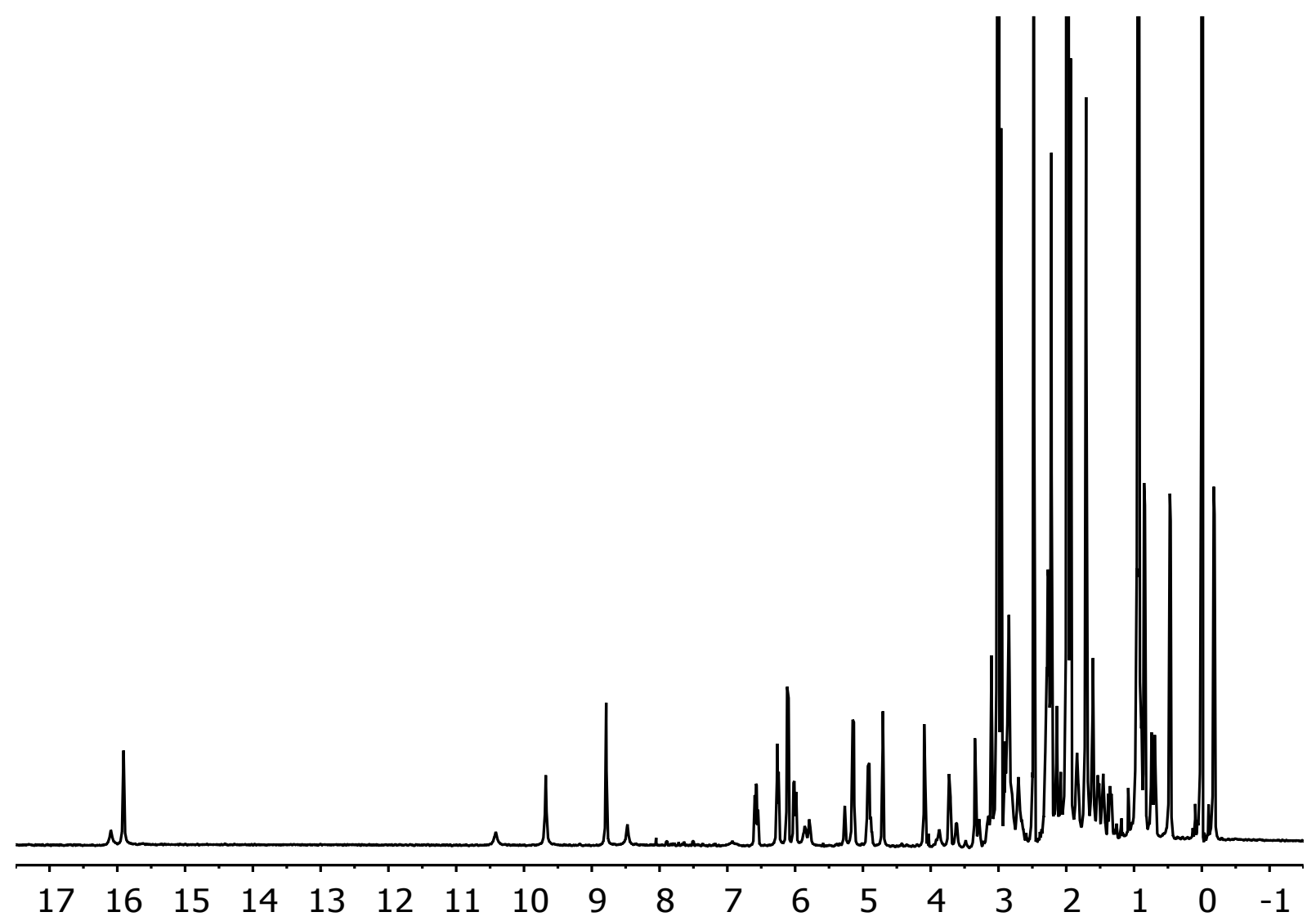


${ }^{1} \mathrm{H}$ NMR spectrum of RFB in $\mathrm{CD}_{3} \mathrm{CN}: \mathrm{DMSO}-\mathrm{d}_{6}+\mathrm{H}_{2} \mathrm{O}$ in $35^{\circ} \mathrm{C}$

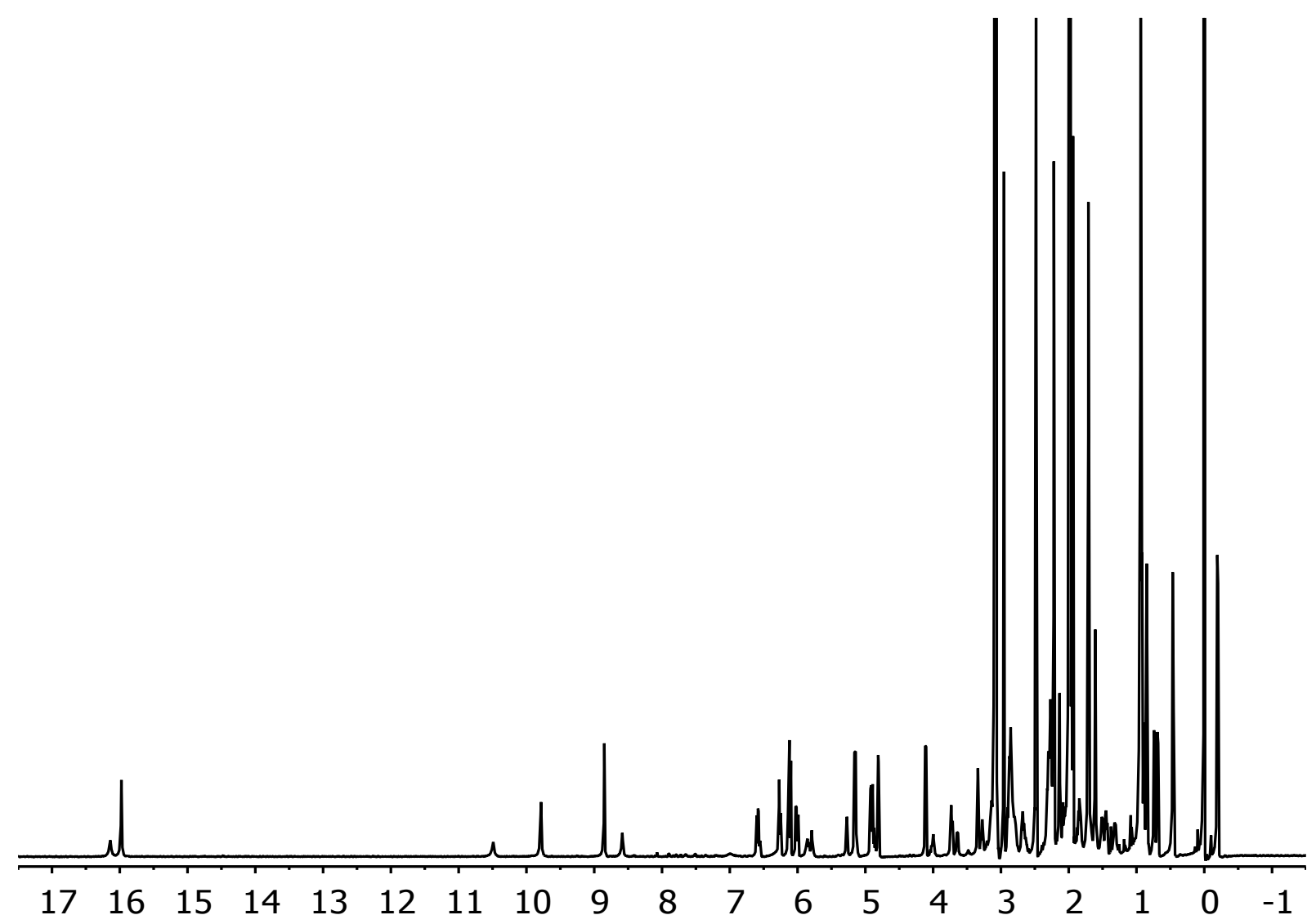


${ }^{1} \mathrm{H}$ NMR spectrum of RFB in $\mathrm{CD}_{3} \mathrm{CN}: D M S O-\mathrm{d}_{6}+\mathrm{H}_{2} \mathrm{O}$ in $25^{\circ} \mathrm{C}$

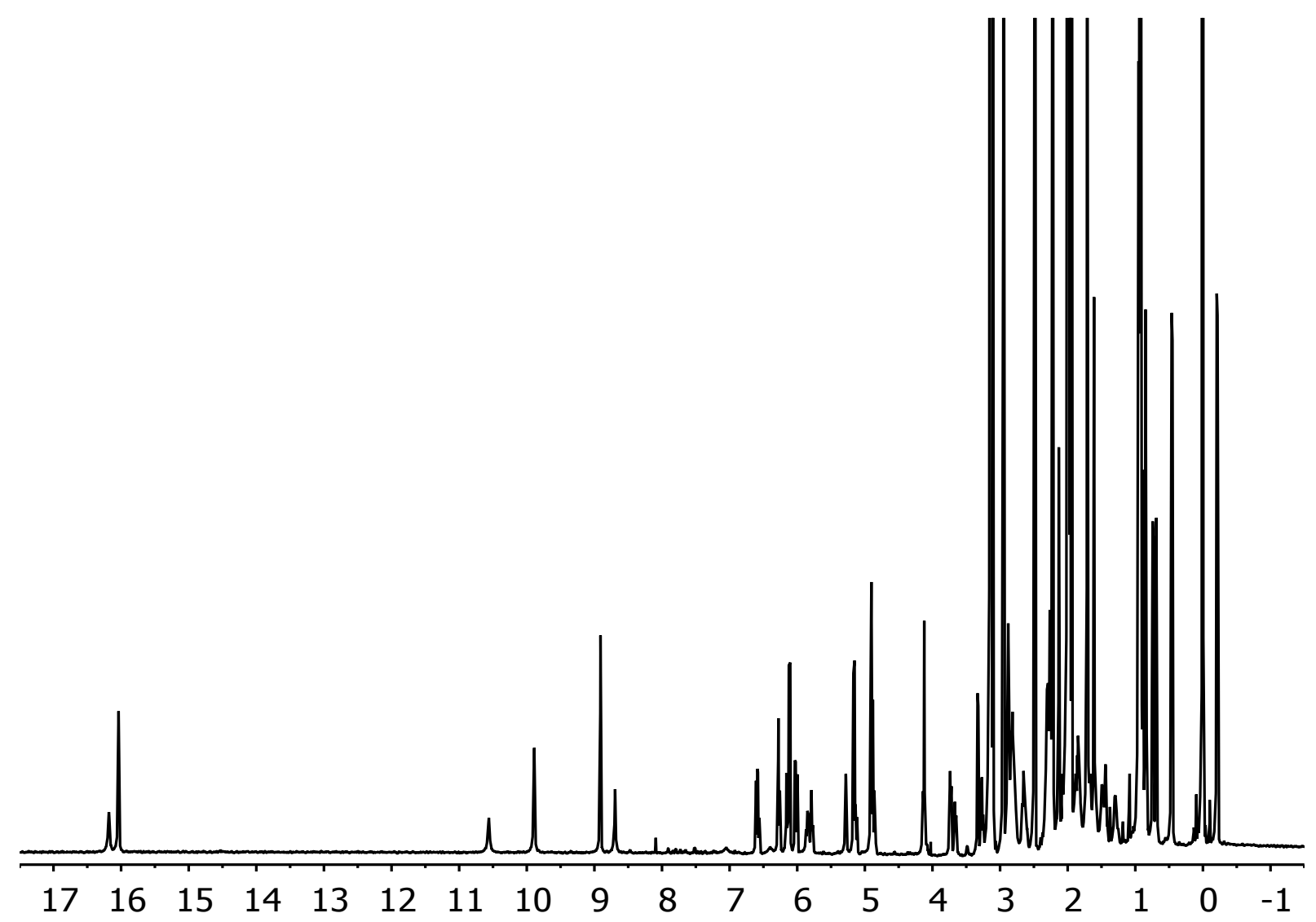


${ }^{1} \mathrm{H}$ NMR spectrum of RFB in $\mathrm{CD}_{3} \mathrm{CN}:$ DMSO- $\mathrm{d}_{6}+\mathrm{H}_{2} \mathrm{O}$ in $15^{\circ} \mathrm{C}$

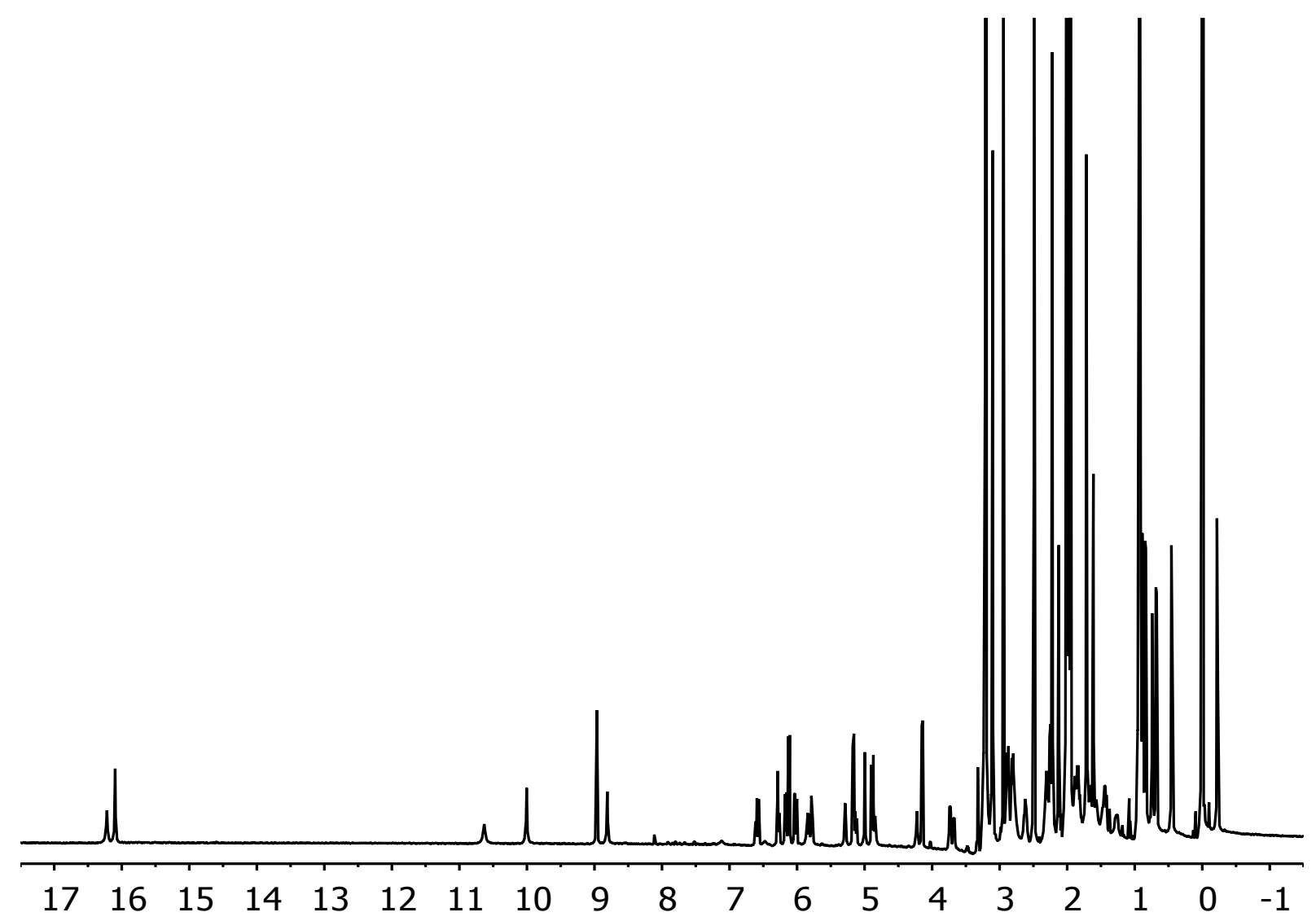


${ }^{1} \mathrm{H}$ NMR spectrum of RFB in $\mathrm{CD}_{3} \mathrm{CN}$ :DMSO- $\mathrm{d}_{6}+\mathrm{H}_{2} \mathrm{O}$ in $5^{\circ} \mathrm{C}$

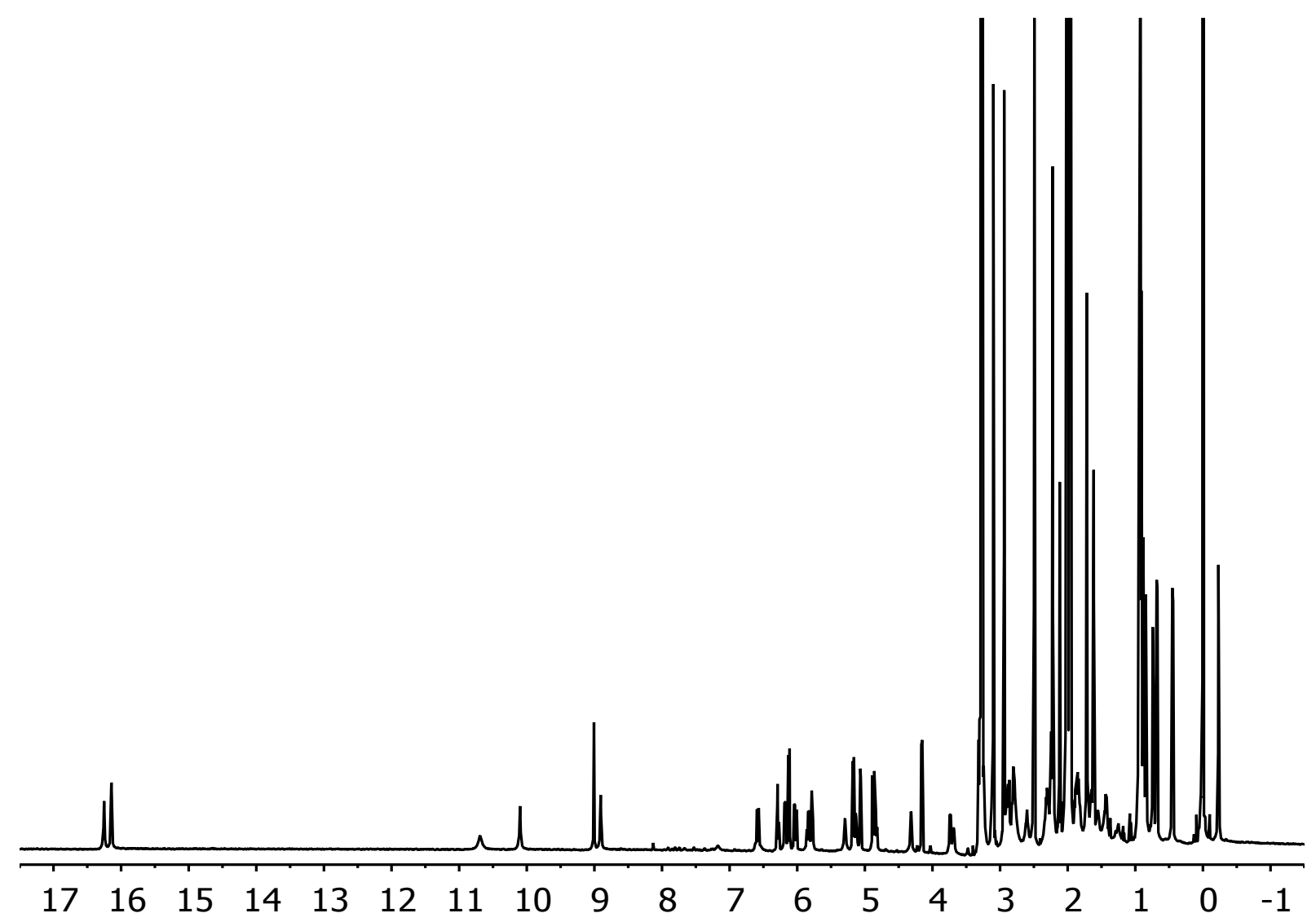


${ }^{1} \mathrm{H}$ NMR spectrum of RFB in $\mathrm{CD}_{3} \mathrm{CN}: \mathrm{DMSO}-\mathrm{d}_{6}+\mathrm{H}_{2} \mathrm{O}$ in $-5^{\circ} \mathrm{C}$

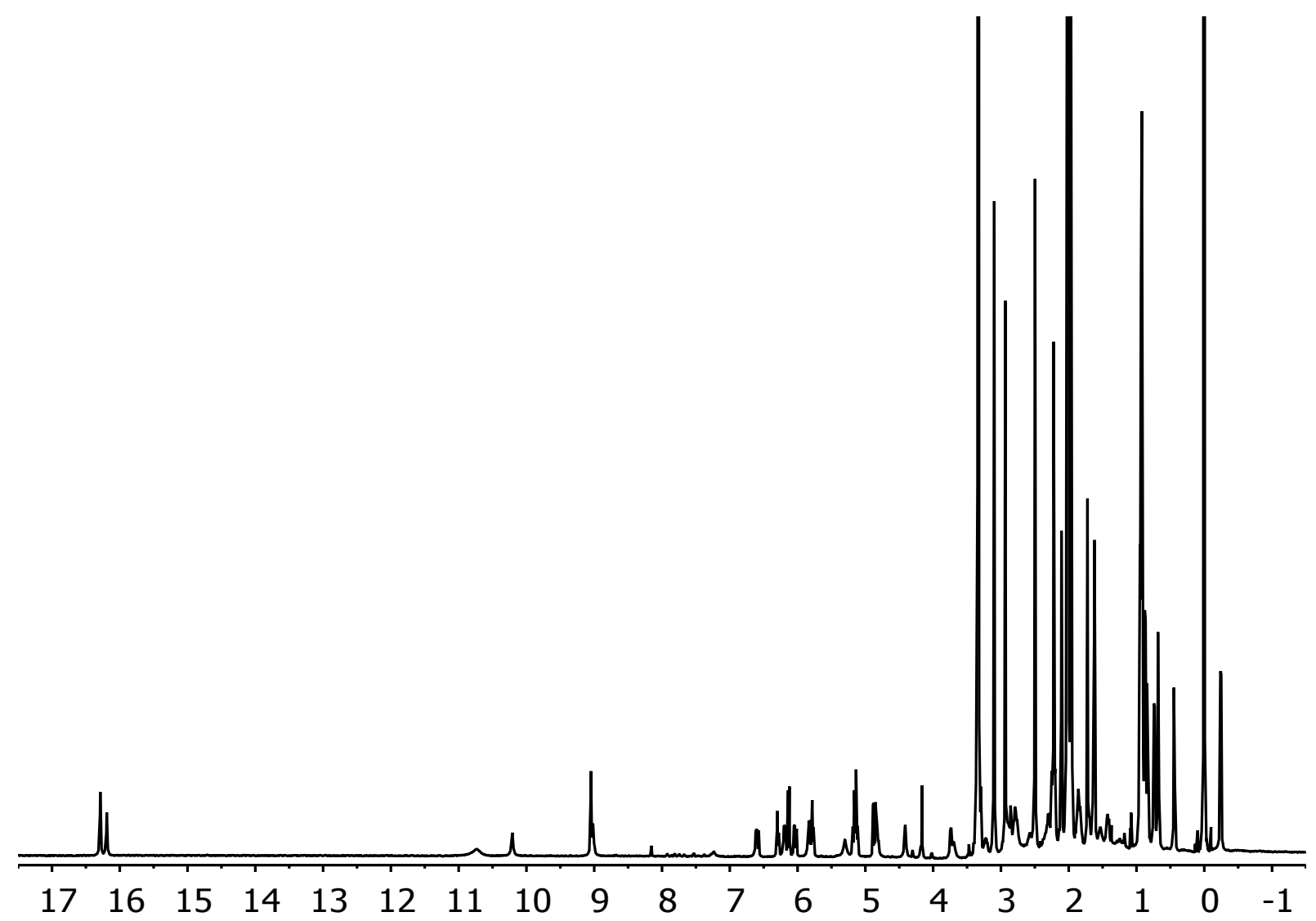


${ }^{1} \mathrm{H}$ NMR spectrum of RFB in $\mathrm{CD}_{3} \mathrm{CN}$ :DMSO- $\mathrm{d}_{6}+\mathrm{H}_{2} \mathrm{O}$ in $-15^{\circ} \mathrm{C}$

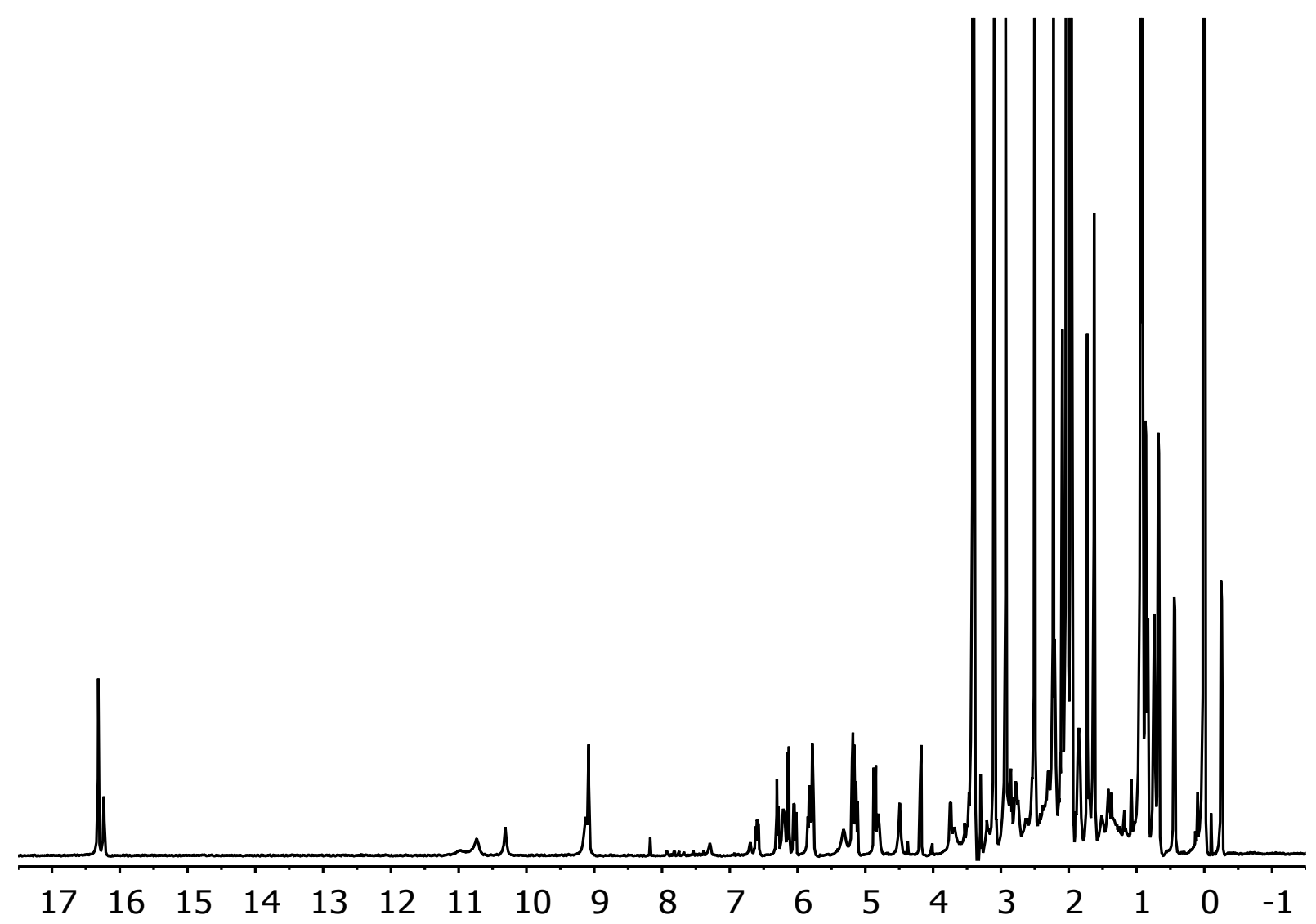


${ }^{1} \mathrm{H}$ NMR spectrum of RFB in $\mathrm{CD}_{3} \mathrm{CN}$ :DMSO- $\mathrm{d}_{6}+\mathrm{H}_{2} \mathrm{O}$ in $-25^{\circ} \mathrm{C}$

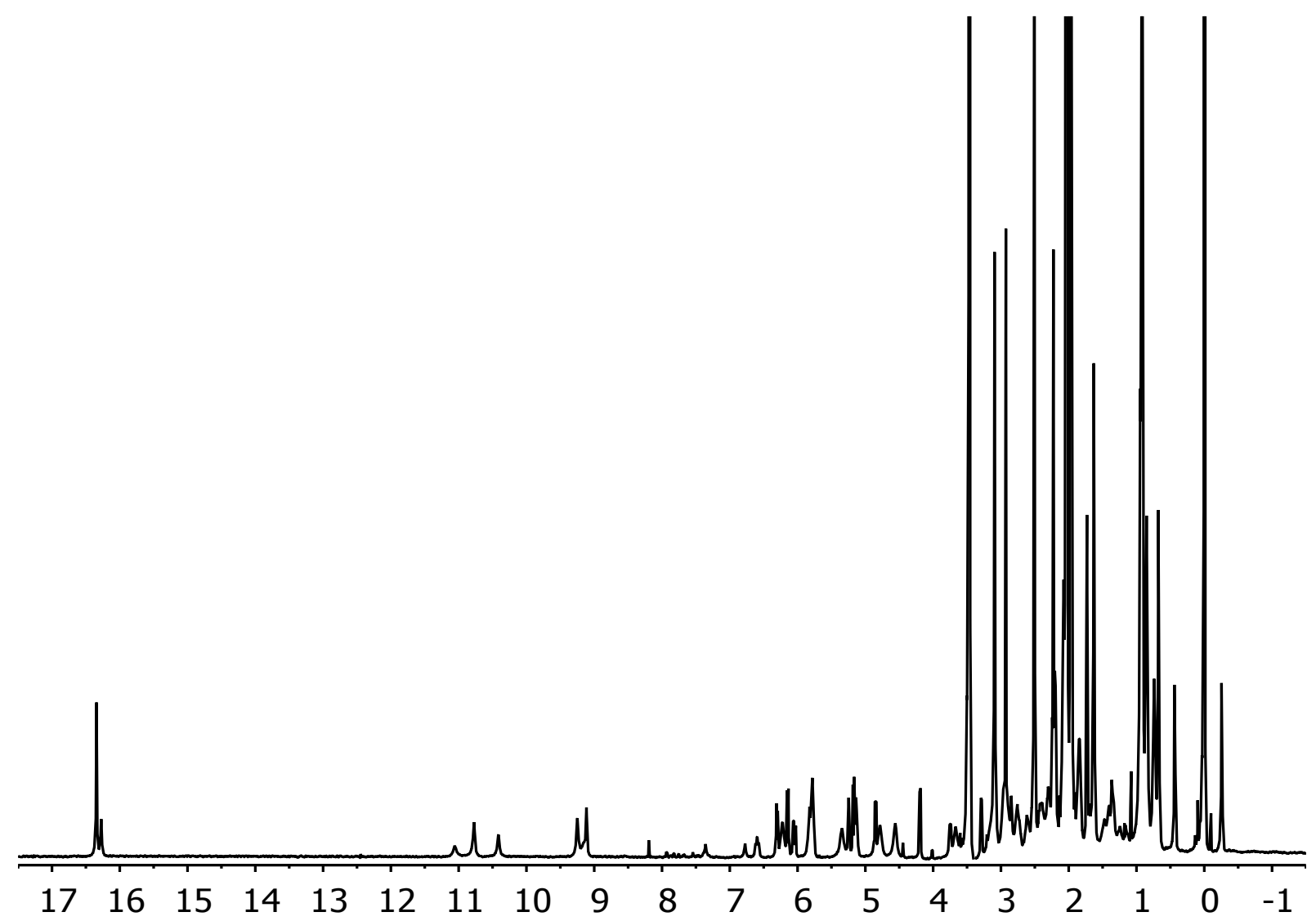


${ }^{1} \mathrm{H}$ NMR spectrum of RFB in Py- $\mathrm{d}_{5}+\mathrm{H}_{2} \mathrm{O}$ in $35^{\circ} \mathrm{C}$

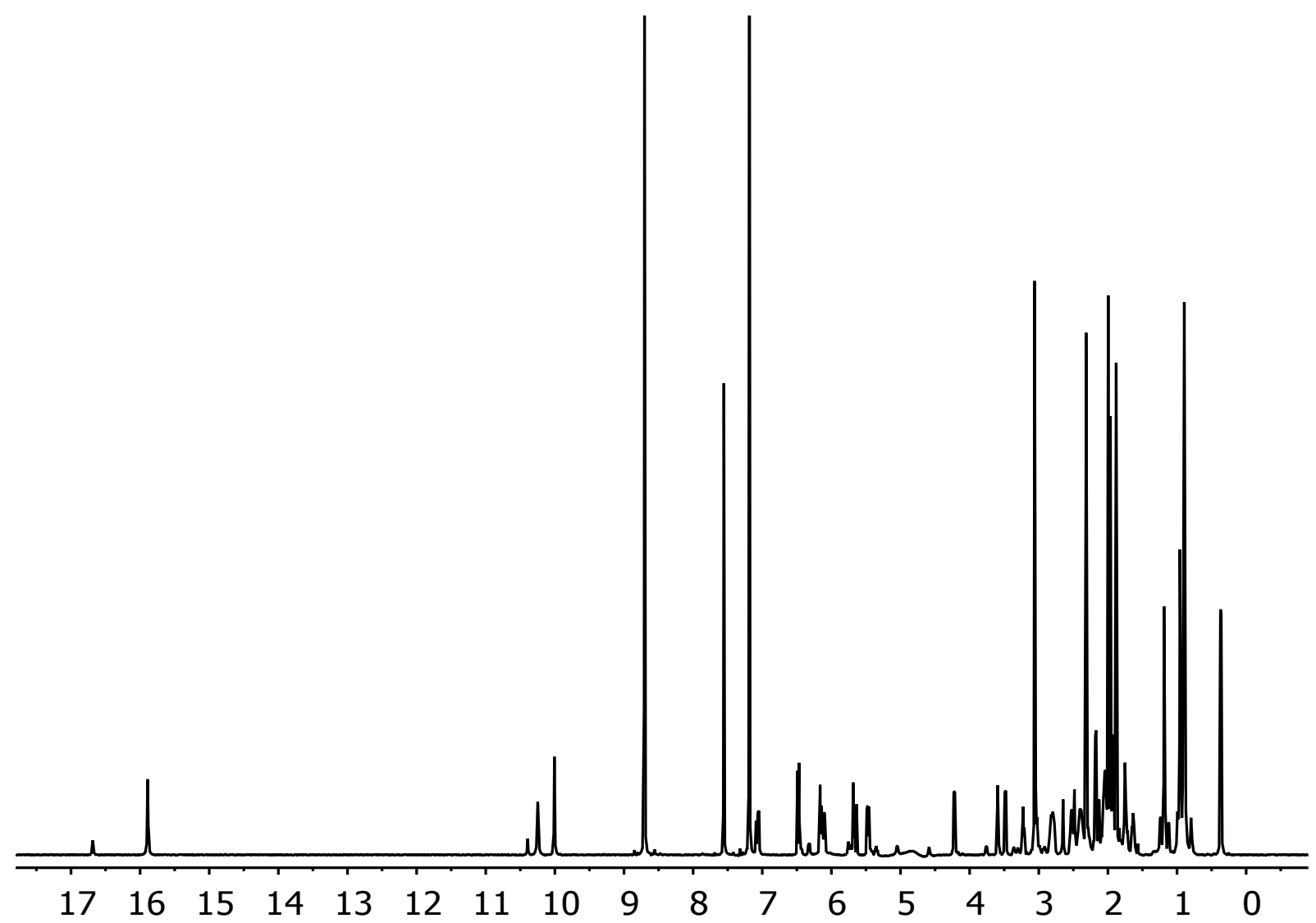


${ }^{1} \mathrm{H}$ NMR spectrum of RFB in $\mathrm{Py}-\mathrm{d}_{5}+\mathrm{H}_{2} \mathrm{O}$ in $25^{\circ} \mathrm{C}$

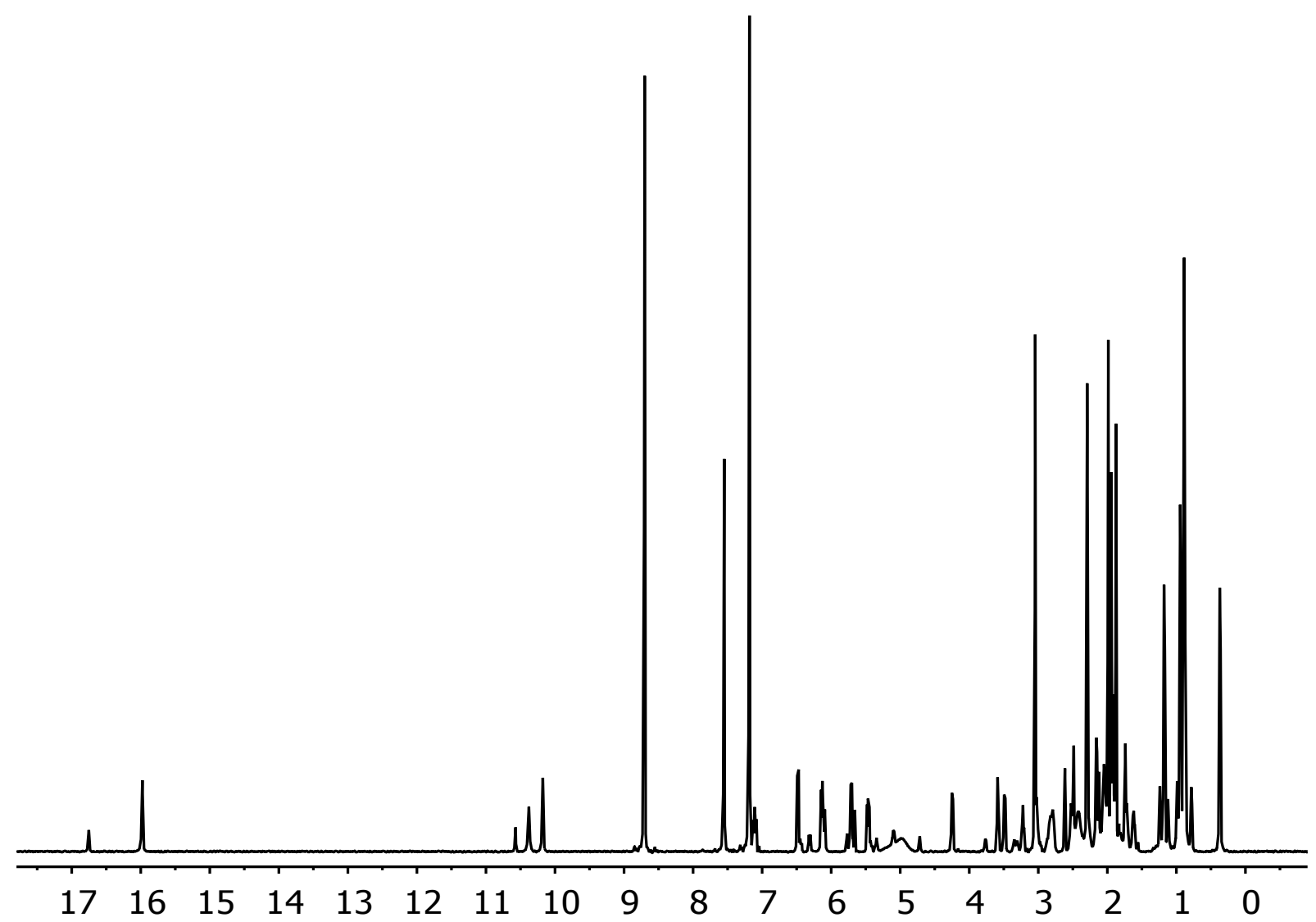


${ }^{1} \mathrm{H}$ NMR spectrum of RFB in Py- $\mathrm{d}_{5}+\mathrm{H}_{2} \mathrm{O}$ in $15^{\circ} \mathrm{C}$

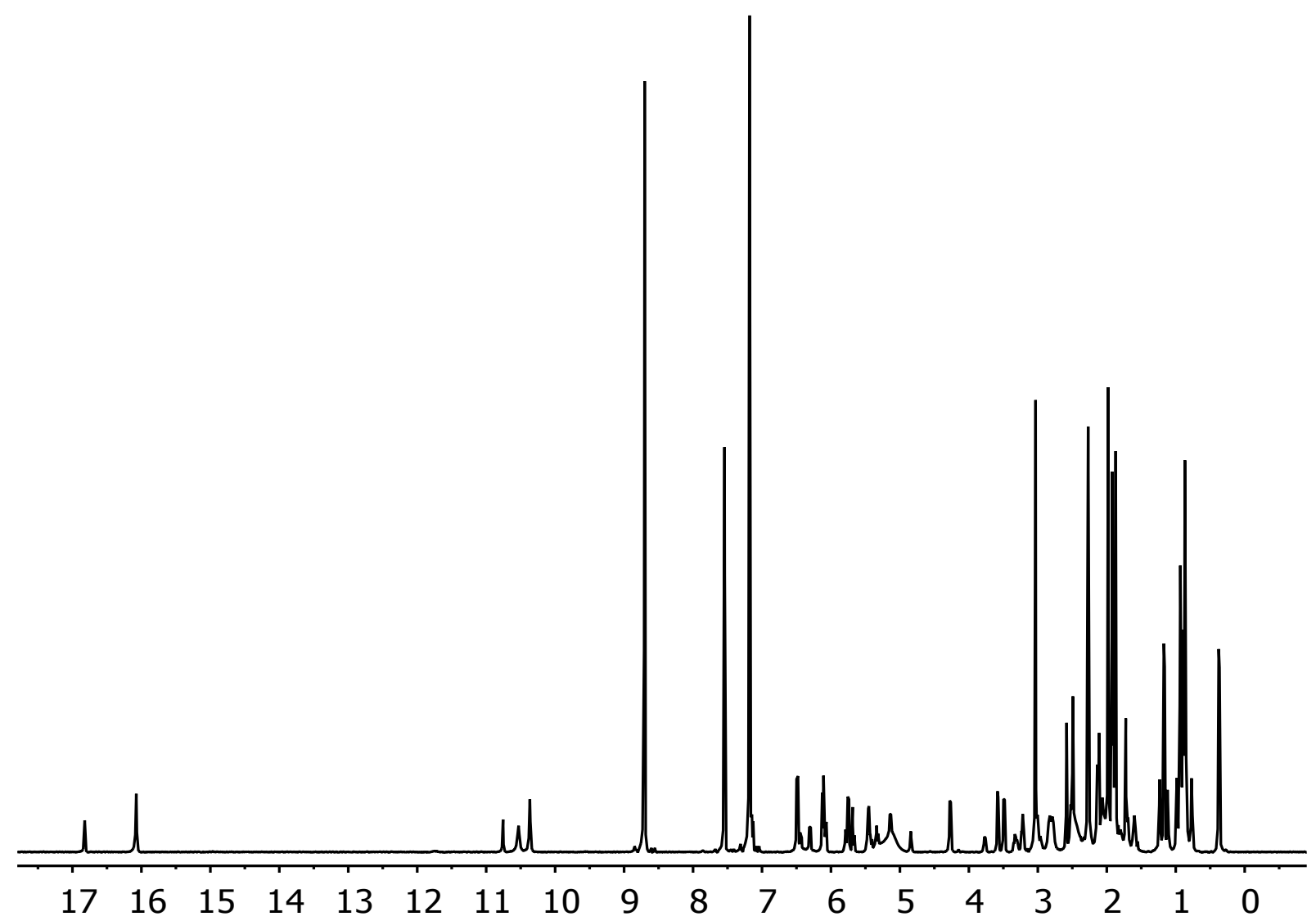


${ }^{1} \mathrm{H}$ NMR spectrum of RFB in Py- $\mathrm{d}_{5}+\mathrm{H}_{2} \mathrm{O}$ in $5^{\circ} \mathrm{C}$

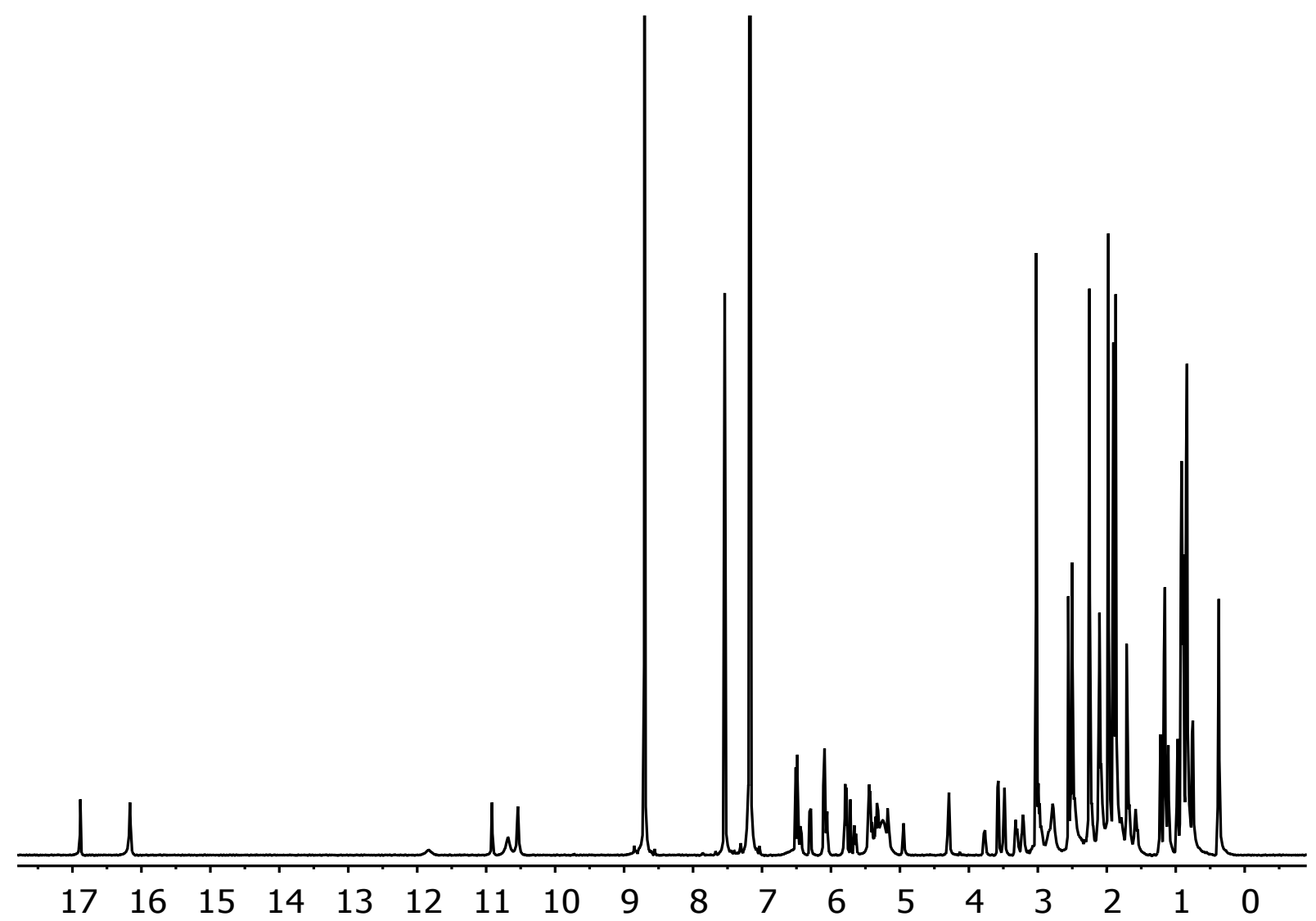


${ }^{1} \mathrm{H}$ NMR spectrum of RFB in $\mathrm{CD}_{3} \mathrm{CN}+\mathrm{H}_{2} \mathrm{O}$

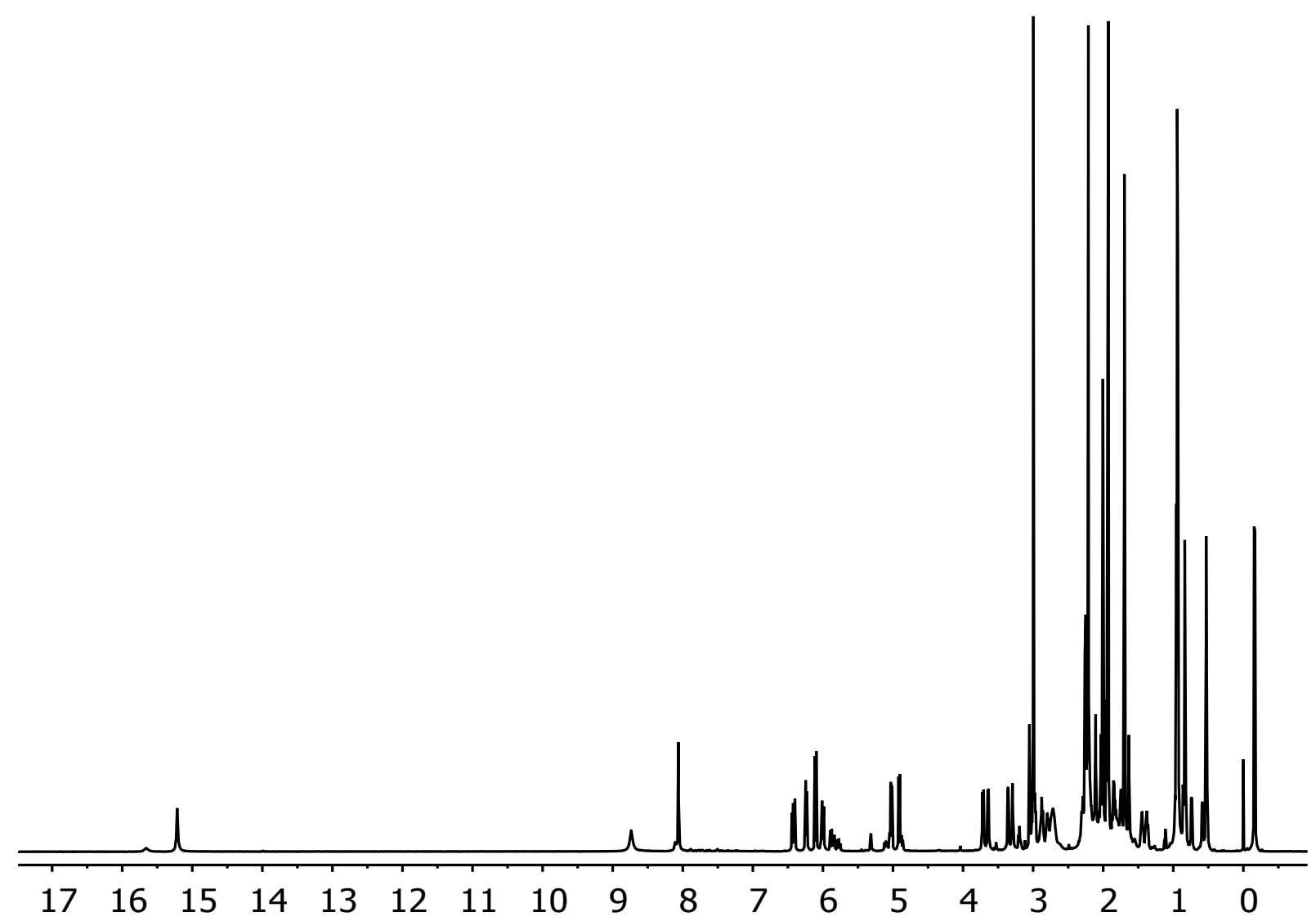


${ }^{1} \mathrm{H}$ NMR spectrum of RFB in $\mathrm{CD}_{3} \mathrm{CN}+\mathrm{H}_{2} \mathrm{O}+\mathrm{H}_{2} \mathrm{O}$

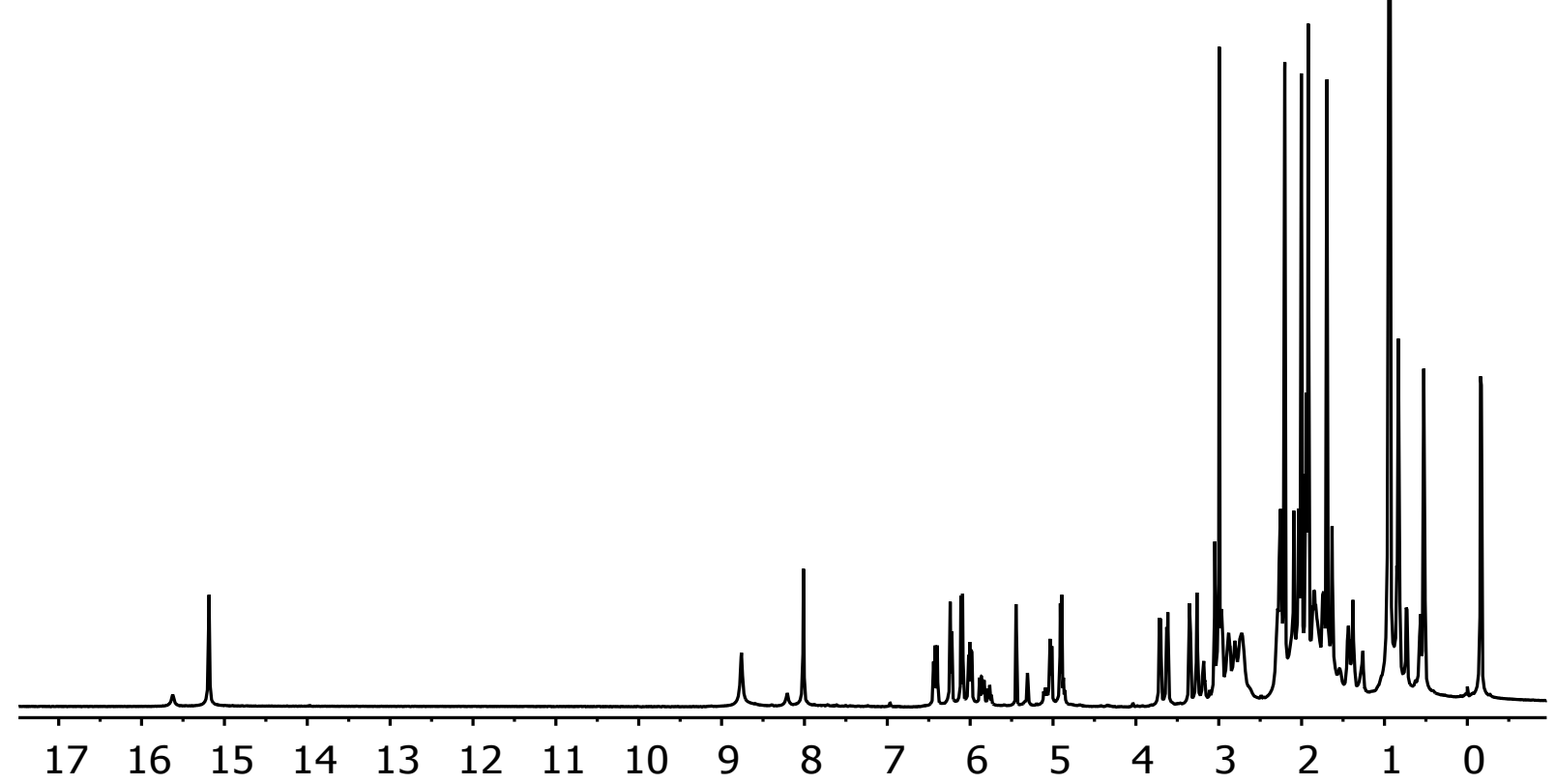


S74

${ }^{1} \mathrm{H}$ NMR spectrum of RFB in $\mathrm{CD}_{3} \mathrm{CN}+\mathrm{H}_{2} \mathrm{O}+\mathrm{D}_{2} \mathrm{O}_{\text {(excess) }}$

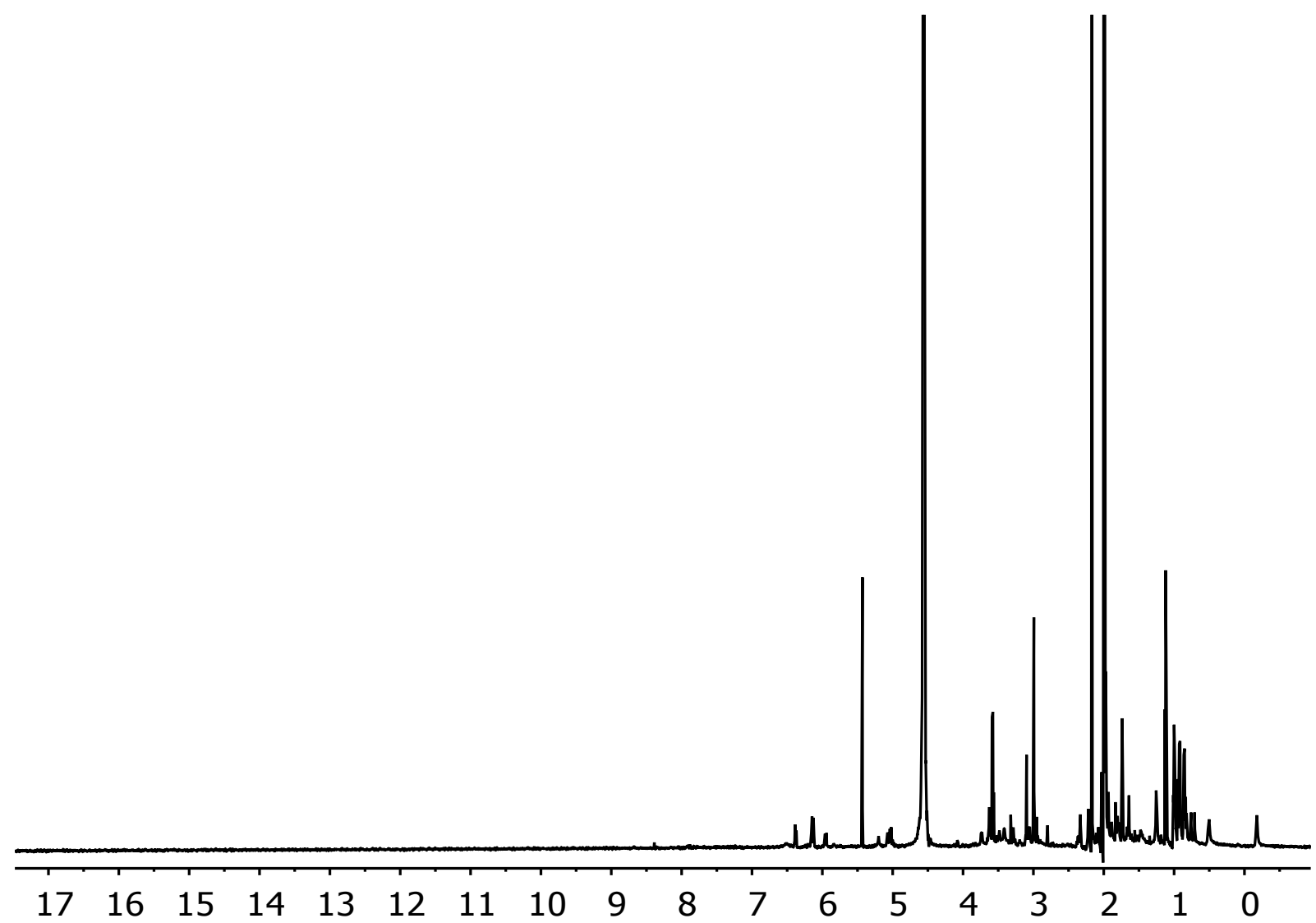


S75

${ }^{1} \mathrm{H}$ NMR spectrum of RFB in DMSO- $\mathrm{d}_{6}+\mathrm{H}_{2} \mathrm{O}$

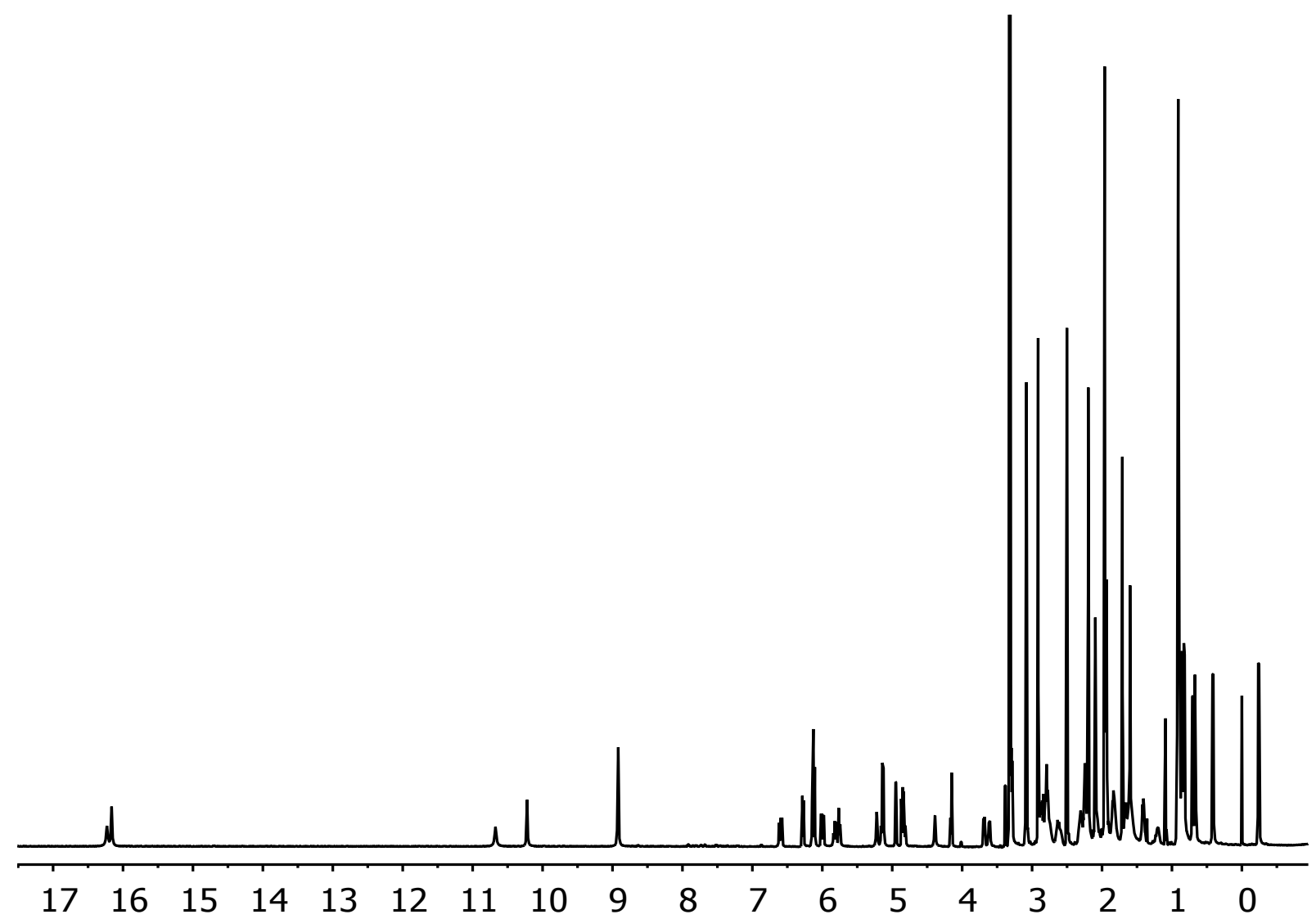


${ }^{1} \mathrm{H}$ NMR spectrum of RFB in DMSO- $\mathrm{d}_{6}+\mathrm{H}_{2} \mathrm{O}+\mathrm{H}_{2} \mathrm{O}$

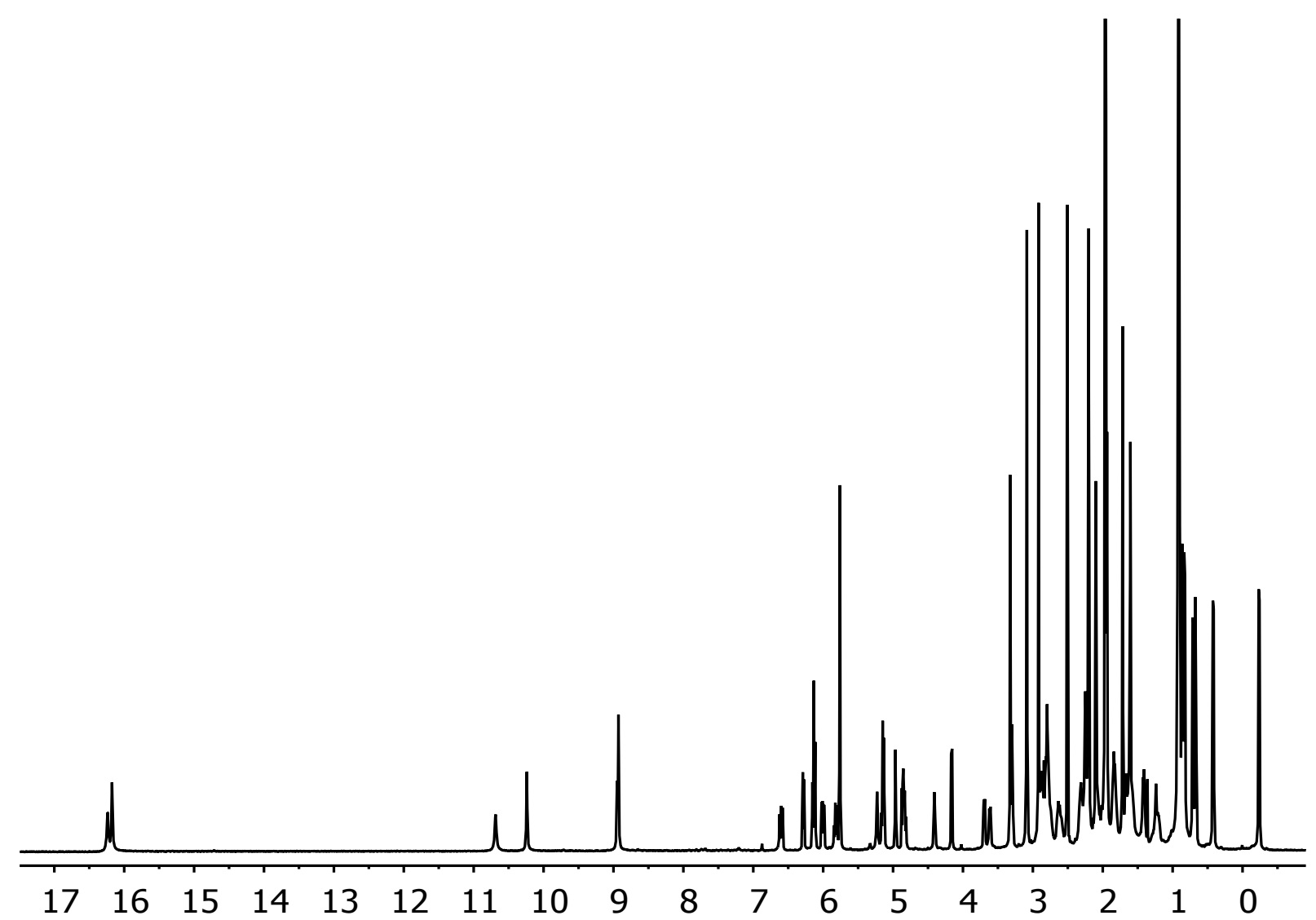


6. HPLC chromatogram

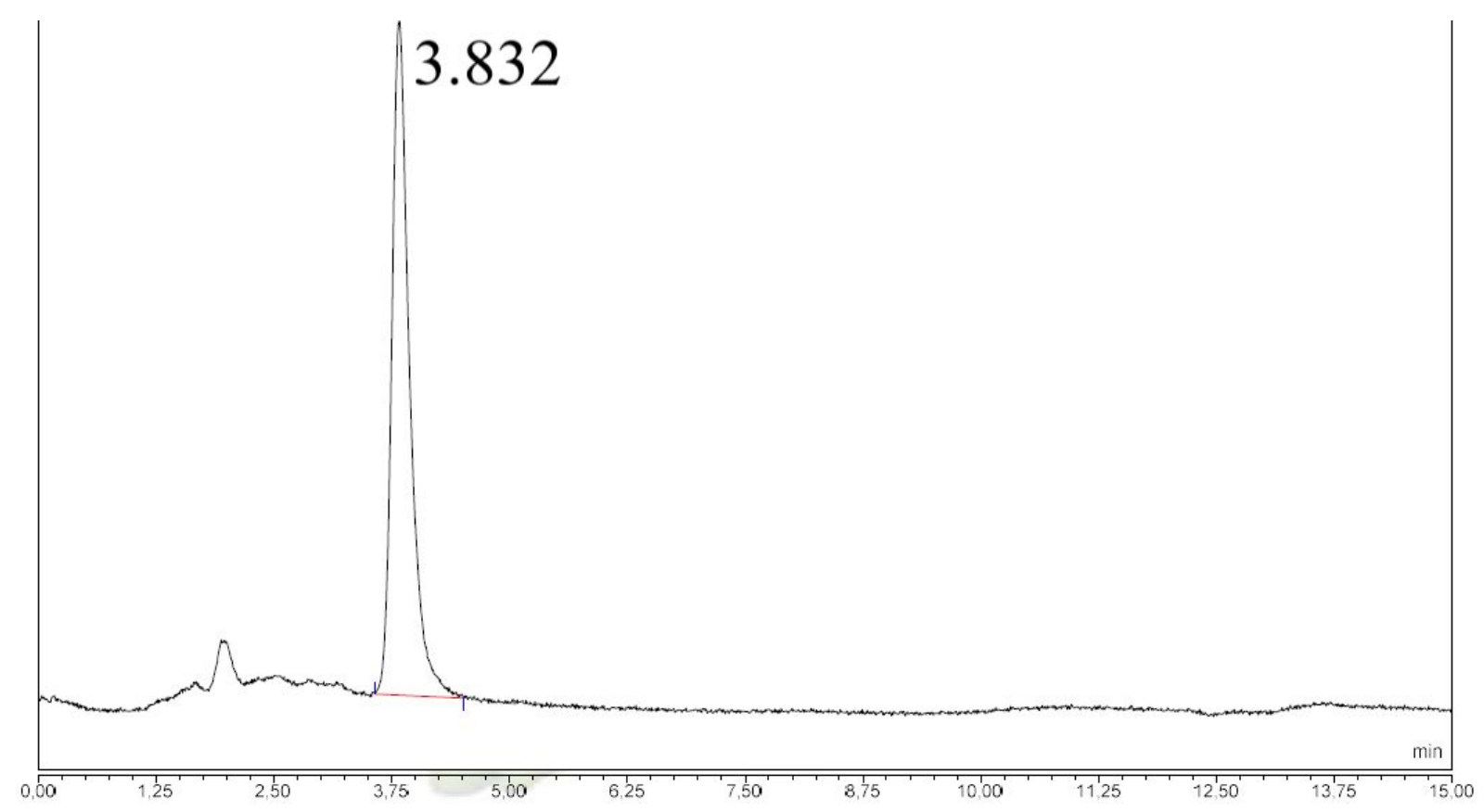

\subsection{RMP chromatogram}

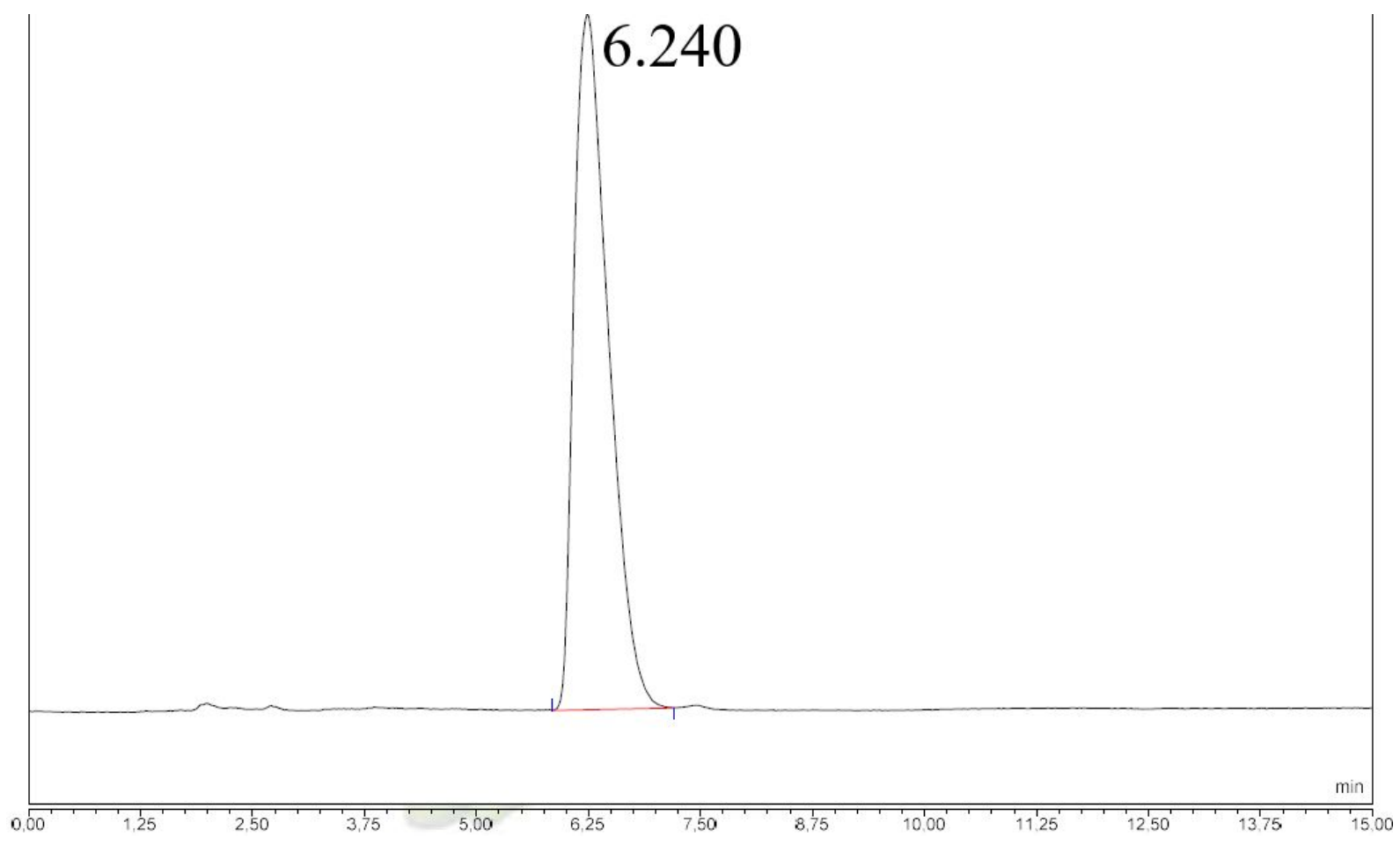

\subsection{RFP chromatogram}




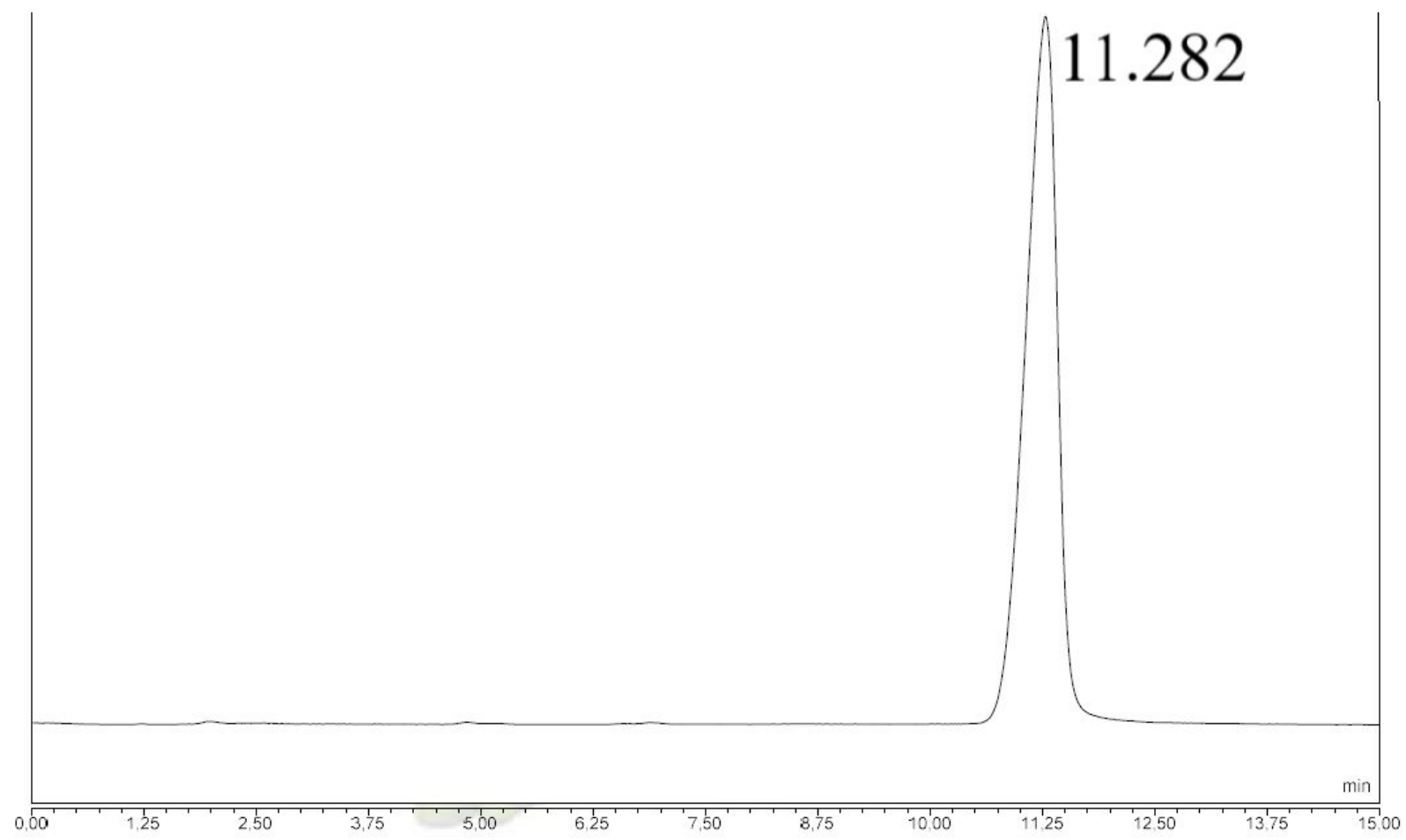

\subsection{RFX chromatogram}

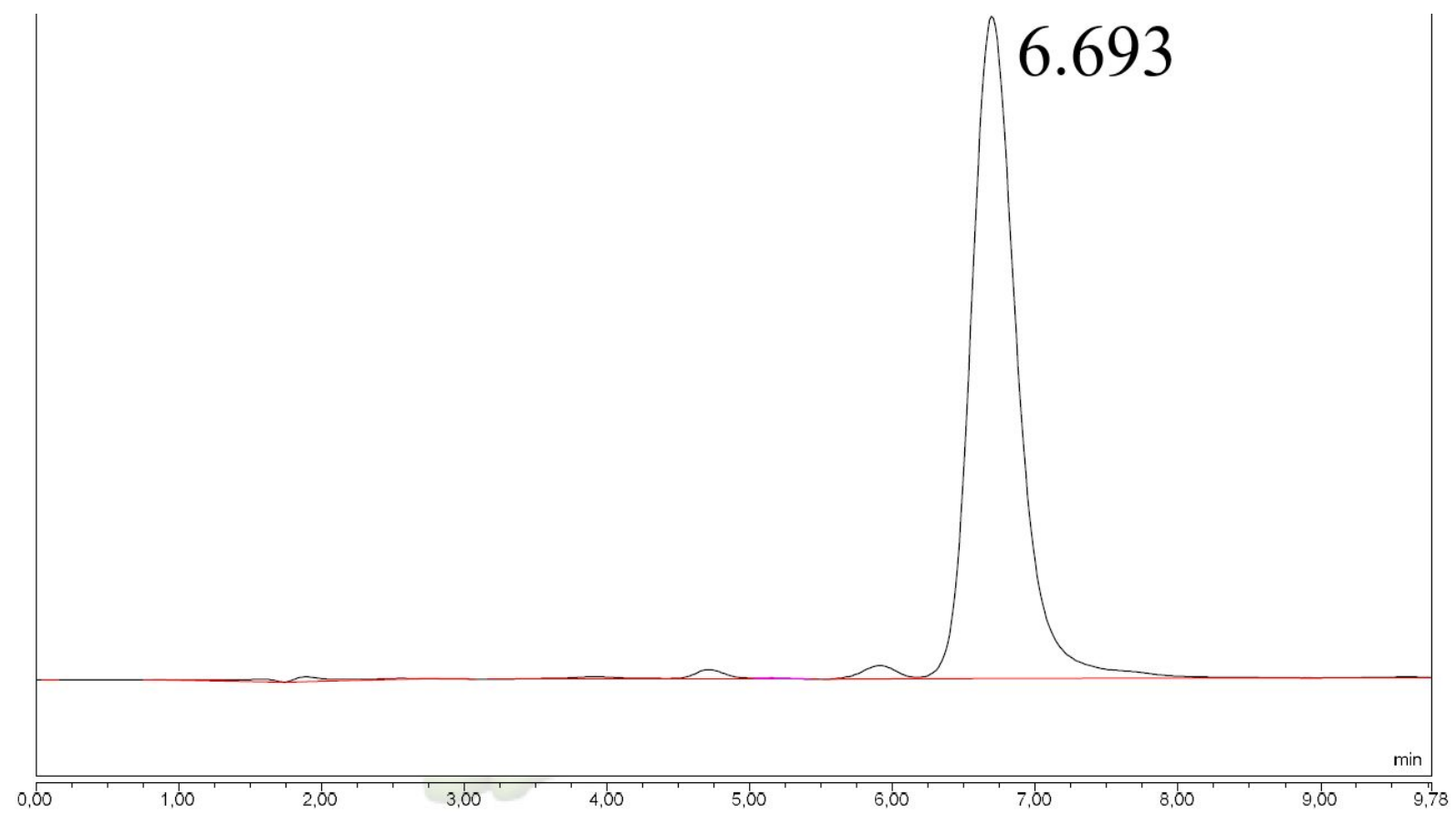

\subsection{RFB chromatogram}

